\title{
PHOTOACOUSTIC EVALUATION OF SURFACES VIA PULSED EVANESCENT FIELD INTERACTION
}

\author{
A Dissertation presented to \\ the Faculty of the Graduate School \\ at the University of Missouri
}

In Partial Fulfillment

of the Requirements for the Degree

Doctor of Philosophy

by

BENJAMIN S. GOLDSCHMIDT

Dr. John A. Viator, Dissertation Supervisor

MAY 2014 
The undersigned, appointed by the Dean of the Graduate School, have examined the dissertation entitled:

\section{PHOTOACOUSTIC EVALUATION OF SURFACES VIA PULSED EVANESCENT FIELD INTERACTION}

presented by Benjamin S. Goldschmidt, a candidate for the degree of Doctor of Philosophy and hereby certify that, in their opinion, it is worthy of acceptance.

\begin{tabular}{c}
\hline Dr. John A. Viator \\
\hline Dr. Heather K. Hunt \\
\hline
\end{tabular}

Dr. Gang Yao

Dr. Scott H. Holan 


\section{DEDICATION}

I dedicate this dissertation to my parents, Michael E. Goldschmidt and Susan R. Santoscoy, without whom I would not be the person I am today. 


\section{ACKNOWLEDGMENTS}

I have been at the University of Missouri for nine years and in that time I have met awesome people that I would like to thank for their contribution to this dissertation. But before I thank those people I would like to write a particularly serious message to all the minimum wage jobs I've ever worked at:

\section{Plaza 900/The MU bookstore/That Quiznos that never hired me even} after offering me a job: Thank you so much for being so financially and emotionally terrible because without you, I never would have realized how much I hate being poor. You have encouraged me to continue my education so that I never have to work for you again. You made me want to quit the University and if I had not felt that way, I never would have worked for Dr. John Viator or created three commercial optical prototypes or created several new fields within photoacoustics. Thank you for being so overwhelmingly horrible. Your lessons will not soon be forgotten.

First, I would like to thank my committee members, John A. Viator, Heather K. Hunt, Gang Yao, and Scott H. Holan, for their support with my unique photoacoustic project. You have shaped my experiences with research while still giving me space to pursue my own interests and my own form of creativity which I will always remember and appreciate.

Dr. Viator: Your constant encouragement and optimism surrounding my ideas for TIRPAS and QTPAS has given me an unbreakable desire to continue work in 
areas that spark my scientific interest. I cannot thank you enough for allowing me to explore ideas that at the time seemed like they would never work. Your willingness to push past the ordinary has given me a body of work that I will always be proud of for the rest of my life without exception. The discussions we have had on statistical principles, mathematical formulae, and business strategies will stay with me for a long time to come. I will never forget the amazing opportunities you have given me, from building three commercial prototypes, to helping me achieve a sizable patent portfolio, to even starting my own biomedical diagnostic business, Red Flag Biosensing LLC. I owe you everything I am in research. If you had not taken the chance on me at the end of the semester in 2007 when I met with you to tell you that I would drop out from the University due to poor grades, being stressed out, and hating working at Plaza 900, I would not have received a B.S. in Biological Engineering and the world would not know about TIRPAS, QTPAS, PAQT, and the power of the photoacoustic effect to change the world in ways both big and small. Thank you for all the years of the Bacon Explosion, thank you for the trust you placed in me from using the procard to training your students, and thank you for all the memories. I feel like in the past few years I have gained more than a mentor. You are and always shall be my friend.

Dr. Heather K. Hunt: Although we met after I had been at the University for several years, I consider your help with my project, both financial and intellectual, to have been crucial to its success. The fact that your lab specifically focuses on evanescent biosensing has greatly helped me push the limits of my photoacoustic/evanescent biosensing/film characterization systems to levels I had not anticipated being possible 
before. Thank you for helping shape the department of Bioengineering for the better by instituting clear manuals for masters and PhD tracks as the process is now much more streamlined and understandable. I am thankful that the last class I took at MU was your materials characterization class because I learned so much I felt like I truly understood what it means to do real science. You have given me many opportunities such as giving my first talk at the Bioengineering Colloquium, writing a small potential grant together, and then an additional talk on Whispering Gallery Mode biosensing at Photonics West. These are things I will never forget that have made me a more effective scientific communicator which is becoming increasingly more important every year. In addition, you have been a very effective sounding board for my research frustrations. Finally, thank you for your advice on future employment, with your help I am sure I will find a job that $99.99 \%$ of my friends and family will have no idea what I actually do.

Dr. Gang Yao: I have taken your classes several times from your research methods class to your biomedical optics class. I owe most of my knowledge regarding basic optics to you. Your teaching style always kept me on my toes as you would seemingly always ask me a question on the few occasions when I did not know what you were talking about. You have instilled in me the quality of asking hard questions even when I know it will take a very long time or a lot of work to answer them. The hard questions with elegant solutions are usually the ones that make all the difference in research. Additionally, thank you for letting me borrow tons of your equipment over the years to complete my research. 
Dr. Scott H. Holan: I audited your class, time series analysis in order to better understand my research with the photoacoustic effect. I have to admit, I think I have a long way to go before I can truly understand time series analysis to the depth that you do, but you have taught me that I do have so much more to learn. Incidentally, your question "How does the error propagate?" with regard to my TIRPAS system has led to extremely important discoveries in my work which led me to conclude the need for an instrumentation amplifier and other components for correct system operation. Thank you for asking the right questions.

Beyond my committee members I have had the pleasure to work with all the great Viator lab and Hunt lab members over the years. In the Viator lab we had the senior members of the laboratory including Kiran Bhattacharyya, Paul Whiteside, and Sagar Gupta.

Kiran Bhattacharyya: You and I have done some awesome things in the labeverything from making a surgical illumination system to solving the research hurdles with PAFC to teaching the undergraduates how not to blind themselves with the Surelite. You inspire my creativity and are the reason why I never felt bored to "just do it" with regards to research. Also, you taught me the very valuable lesson of "going incognito" to get research done. Thank you for all your insight and your friendship.

Paul Whiteside: We have developed a truly unique system with PAQT that I hope changes the way dermatologists perform optical ablation for years to come. Over the years you have been my office mate, room mate, and drinking buddy, but research- 
wise you have always been my contrarian which has helped me develop substantial scientific communication skills since I have to be even more convincing when I talk to you about a new idea. Despite our different approaches to research I have no doubt you will succeed and I look forward to collaborating with you for many years to come.

Sagar Gupta: You have known me the longest at the lab other than Dr. Viator and I want you to know that most of what I learned in terms of running the lab, PAFC, and exploring possibilities to fix engineering problems I learned from you. I still remember all the time you spent trying to teach me how to align the optical detector and while I do think piezoelectric transducers are 1000 times easier to use overall, I did have some measure of fun despite the long nights. Thanks for all your expertise and care.

Carol Soteropulos: Thank you for telling me how much I am valued at the Viator lab/Hunt labs. I really appreciated your perspective/advice on a great many things from research to life. I will not pretend to understand how hard it was for you to pull a taper when the spool was acting up, but I will admit, it was fun having you around even when the taper would break seven times in a row. I will miss the McNally's Mondays and the Midweek Madness with you. I wish you the best of luck in achieving your dream of becoming a doctor.

Jeremy Dahmen: I can not even begin to understand your unnatural love of Taco Johns, but we always had a lot of fun in the kitchen bothering Carol. Every time I think of the stories you told me about yourself I can't help but laugh as they 
are all hilarious and I want to thank you for sharing as they got me through some frustrating research questions. Also, like I wrote previously, I will not pretend to understand the trials and tribulations of breaking concurrent tapers, but I will say it was great having you in lab to hang out when that did happen.

Swarnasri Mukherjee: Thank you for all the help you have given me with understanding ellipsometry, finding film properties, purchasing materials for TIRPAS, and always lending me a helping hand when I need it. Additionally, thank you for the random talks we have had in the kitchen regarding research and life in general. Your perspective always makes me rethink the way I look at the world.

Emily O'Brien: Thank you for sitting there with me when I was troubleshooting the new biosensing program I made in Labview. Aside from the great deal of troubleshooting we got done, I know that your insight into WGM biosensing has really helped me understand another fundamental method of evanescent biosensing which will only add to the thorough nature of this dissertation.

Several extraordinary undergraduates such as Christine O'Brien, Amanda Sudduth, and Jeff Mosley deserve special mention as well.

Christine O'Brien: You truly kickstarted PAFC and gave me the resources needed to continue the work to create a commercial prototype. We have had our share of disagreements, but you have always been helpful and a great research colleague. I am continually impressed with your ability to plan and lead research projects, out- 
ings, and important events. Someday you are going to inspire students to change the world and give them the tools they need to truly excel in this world. Keep up your work, you are going to be great.

Amanda Sudduth: You were my first undergraduate that I ever got to train on my dissertation work with TIRPAS. You were truly instrumental in helping me get my PhD. Without your help with physically doing the experiments (like turning the stepper motor dials for 6 hour spans of time) modern TIRPAS would never have been published never mind the additional work of QTPAS or PAQT as well. I am glad that you were my first undergraduate. You are brilliant and I am proud to have trained you.

Jeff Mosley: You brought a practical perspective to the laboratory in the form of Labview expertise and circuitry knowledge. You are the reason why I know Labview so well and I truly appreciate that since it is so integral to getting QTPAS and TIRPAS to work. We have been on a patent together and we have worked on building the commercial CTC prototype. All in all, it has been a blast getting to work together on these projects as you always have a good way of looking at things to get them to truly work.

I would like to thank my collaborators as well in the Boone County Lumber Building Technology Laboratory within the Department of Architectural Studies at the University of Missouri. 
Bobbi J. Hauptmann: Thank you for machining various components for me over the years for my research and various projects. Honestly, without you and your willingness to put in extra hours on the Versa Laser or machining baseplates my $\mathrm{PhD}$ would not have been a success. You were a pleasure to work with and I will miss our talks about research, life, and the future. You helped me create a new type of photoacoustic biosensing that will have applications for years to come. Thank you so much for all you have done.

Ronald G. Phillips: Thank you for giving me access to the laboratory. Without that I would not have been able to finish my $\mathrm{PhD}$ without a significant amount of extra effort. I sincerely hope that engineering and Human Environmental Design continue to collaborate as I believe our collaboration truly made a difference in my $\mathrm{PhD}$ and as I mentioned above helped create a new type of photoacoustic biosensing. Have fun with your retirement years with the knowledge that you have helped generations of my family succeed academically.

Gordon Ellison: Thank you for all your help with design, machining, and advice on my projects over the years. Thank you so much for designing everything from a prototype light source to a cancer detection system to TIRPAS and QTPAS components. You single handedly improved the safety of the lab (laser light beam dumps), improved the sensitivity of my TIRPAS device by a factor of 10 (TIRPAS mount), and helped me meet critical deadlines for demanding collaborators. Gordon, you are awesome, thank you. 
I would like to thank a few undergraduates/high school students that worked directly with me.

Ben Robertson: I know what you are thinking, but no, I won't write that in the dissertation, I will only say it to you when Adam Eshein and Kiran Bhattacharyya are around to laugh. Seriously though, you are a great researcher and I hope you are having a great time in Texas.

Rebecca Burke-Agüero: You truly are exceptional. Keep up the great work at Vanderbilt and someday you are going to win a Nobel prize. Do not forget your roots. You will forever be a Viator lab student.

The rest of the undergraduates: As much as I would give all of you trouble regarding professionalism, hard work, doing basic math, and not blinding yourselves with the Surelite I-20, I will say that it was a pleasure working with each of you. You have all greatly exceeded my expectations and are going to be competent critical thinking scientists. Congratulations on being exceptional, you made my experience at the Viator lab one I will never forget.

Mr. Lynn D. Johnson: Thank you for your assistance regarding the Labview program I created for evaluating thin films using QTPAS. Without your help I'd still be doing the characterization manually which wouldn't have been very fun at all.

Benjamin Watkins and Cristin Thompson-Watkins: Thank you both so 
much for being supportive friends during my extended education. You are wonderful people and you definitely helped me get through this challenging time of my life.

David E. Casper: Now that you are pursuing a computer science degree I have a special binary message for you. "01000110011011110110000101101101" 


\section{TABLE OF CONTENTS}

ACKNOWLEDGMENTS ................ ii

LIST OF TABLES $\ldots \ldots \ldots \ldots \ldots \ldots \ldots$ xviii

LIST OF FIGURES $\ldots \ldots \ldots \ldots \ldots \ldots \ldots \ldots \ldots \ldots \ldots \ldots \ldots$

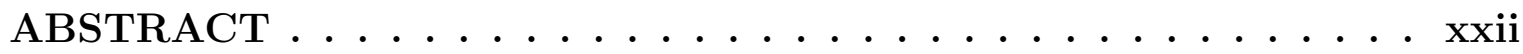

\section{CHAPTER}

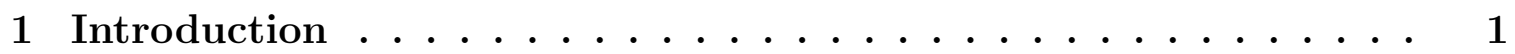

1.1 Mesoporous Materials . . . . . . . . . . . . . . . . . . 2

1.2 Current Techniques . . . . . . . . . . . . . . . . 3

1.2.1 Transmission Electron Microscope . . . . . . . . . . . . . . 4

1.2.2 Scanning Electron Microscope . . . . . . . . . . . . 5

1.2 .3 Ellipsometry . . . . . . . . . . . . . . . . 7

1.2.4 Atomic Force Microscopy . . . . . . . . . . . . . . . . 8

1.3 The Photoacoustic Effect . . . . . . . . . . . . . . . . . . 11

1.3.1 Biomedical Applications of the Photoacoustic Effect . . . . . 12

1.4 Total Internal Reflection Photoacoustic Spectroscopy _. . . . . . . . 12

2 TIRPAS and SPRPAS $\ldots \ldots \ldots \ldots \ldots \ldots \ldots$

2.1 Total Internal Reflection . . . . . . . . . . . . . . . . . . 14

2.1.1 The Evanescent Field . . . . . . . . . . . . . . . . . . . 16

2.1 .2 Photoacoustic Effect . . . . . . . . . . . . . . . 22 
2.2 Total Internal Reflection Photoacoustic Spectroscopy $\ldots . . . . . .26$

2.2.1 Surface Plasmon Resonance Photoacoustic Spectroscopy _. . 28

2.2.2 Modern TIRPAS with Q-switched Lasers . . . . . . . . . . 29

3 Modern TIRPAS and Biosensing . . . . . . . . . 30

3.1 Evanescent Sensing . . . . . . . . . . . . . . . . . . . . . . 31

3.1 .1 Malaria Analytes . . . . . . . . . . . . . . . . . . . 32

3.2 Materials and Methods . . . . . . . . . . . . . . . . . . 34

3.3 Experimental Setup . . . . . . . . . . . . . . . . . . . . . . . 34

3.3 .1 Laser and Optical Fiber . . . . . . . . . . . . . . . . 34

3.3.2 Sample Holder and Transducer Electronics . . . . . . . . . 35

$3.3 .3 \quad$ Ultrasonic Detector . . . . . . . . . . . . . . . . . . . 36

$3.3 .4 \quad$ Spatial Filter . . . . . . . . . . . . . . . 36

3.3.5 TIRPAS and $\beta$-Hematin $\ldots \ldots \ldots \ldots \ldots \ldots$

$3.3 .6 \quad \beta$-Hematin Synthesis _ . . . . . . . . . . . . . 38

3.3.7 Critical Angle Analysis . . . . . . . . . . . . . . . . . . . . 39

3.3.8 Angular Spectrum: Chlorazol Black . . . . . . . . . . . . . 40

3.3.9 Detection Limit of Chlorazol Black . . . . . . . . . . . . 41

3.3 .10 Data Collection . . . . . . . . . . . . . . . . . . . 44 41

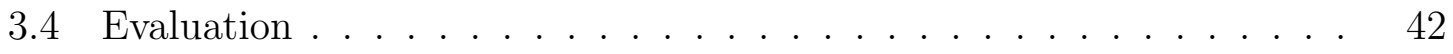

3.5 Discussion . . . . . . . . . . . . . . . . . . . . . . 45

3.5.1 How to Relate Optical Absorption to Refractive Index _. . . 45

3.5.2 Optical Absorption Under TIRPAS Excitation . . . . . . . . 46 
3.5.3 Acoustic Plane Waves in TIRPAS . . . . . . . . . . . . . . 48

3.5.4 Optical Penetration Depth . . . . . . . . . . . . . . . . 49

3.5.5 Stress Confinement . . . . . . . . . . . . . . . . . 50

3.5 .6 Signal Acquisition . . . . . . . . . . . . . . . . . 52

3.5.7 Differences in Penetration Depth . . . . . . . . . . . 54

3.5 .8 Advantages of TIRPAS . . . . . . . . . . . . . . . 54

3.5.9 Optical Scattering of $\beta$-Hematin . . . . . . . . . . . 55

3.6 Conclusion . . . . . . . . . . . . . . . . . . . . . . . . 57

4 Photoacoustic Refractometry (PAS/TIRPAS) and Biosensing . . 58

4.1 Introduction . . . . . . . . . . . . . . . . . . . . 59

4.1 .1 Reflectometry . . . . . . . . . . . . . . . 60

4.1 .2 Critical Angle Analysis . . . . . . . . . . . . . . . . . 60

4.1 .3 Ellipsometry . . . . . . . . . . . . . . . . . 60

4.1.4 Limitations . . . . . . . . . . . . . . . . . . 61

4.1.5 The Photoacoustic Effect . . . . . . . . . . . . . . . 62

4.1.6 Total Internal Reflection Photoacoustic Spectroscopy . . . . . 63

4.1.7 Surface Plasmon Resonance Photoacoustic Spectroscopy . . 67

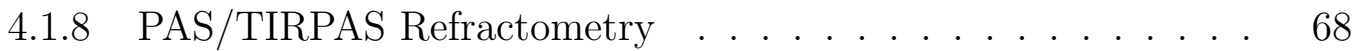

4.2 Materials and Methods . . . . . . . . . . . . . . . . . . . . 69

4.2.1 Direct Red 81 Dye Solutions . . . . . . . . . . . . . . . . . . 69

4.2 .2 Myoglobin Solution . . . . . . . . . . . . . . . . . . . . . 69

4.2.3 PAS/TIRPAS Refractometer . . . . . . . . . . . . . 69 
4.2.4 Laser Leveling . . . . . . . . . . . . . . . . . . . . 70

4.2.5 Labview Automation . . . . . . . . . . . . . . . . . . . 71

4.2 .6 Exploratory Analysis . . . . . . . . . . . . . . . 71

4.2 .7 PAS/TIRPAS Measurement . . . . . . . . . . . . . 72

4.2.8 Optical Refractometer Measurement _. . . . . . . . . . 72

4.3 Results . . . . . . . . . . . . . . . . . . . . . 73

4.4 Discussion . . . . . . . . . . . . . . . . . . . . . 75

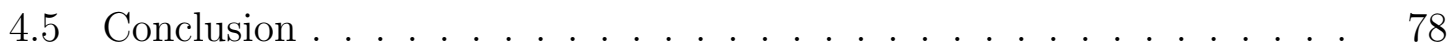

5 QTPAS and Thin Films ................ 79

5.1 Photoacoustic Evaluation of Thin Films _ . . . . . . . . 80

5.2 Quantum Tunneling Photoacoustic Spectroscopy . . . . . . . . . . 80

5.2 .1 QTPAS Derivation . . . . . . . . . . . . . . 83

5.2.2 Assumptions/Limitations of the QTPAS Derivation . . . . . 85

5.3 Labview Automation: QTPAS.vi _ . . . . . . . . . . . . 89

5.3.1 Refractive Index and Thickness Estimation . . . . . . . . . . 91

5.4 Exploratory Analysis and Simulated Example . . . . . . . . . . . 91

5.4 .1 Substrate Scans . . . . . . . . . . . . . . . . . . . . 92

5.4 .2 Laser Energy Interaction . . . . . . . . . . . . . . . . 94

6 Future Work ...................... 96

6.1 Birefringence Determination . . . . . . . . . . . . . . . 96

6.2 Optically Resonant TIRPAS . . . . . . . . . . . . . . . 97

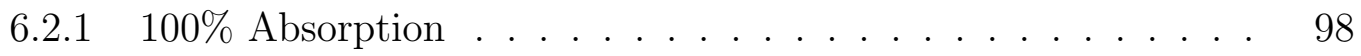


6.3 Porosity Measurements . . . . . . . . . . . . . . . . . . . . 99

6.4 Optical Detection . . . . . . . . . . . . . . . . 100

6.5 Absorbing Thin Films . . . . . . . . . . . . . . 100

7 Summary and Concluding Remarks ............. 102

7.1 Modern TIRPAS and Biosensing . . . . . . . . . . . . 103

7.2 Photoacoustic Refractometry (PAS/TIRPAS) and Biosensing . . . . . 104

7.3 QTPAS and Thin Films ................ 105

7.4 Conclusion ........................... 105

\section{APPENDIX}

A Various Important Issues . . . . . . . . . . . 107

A.1 Electrical Impedance Matching . . . . . . . . . . . . . . . 107

A.2 Acoustic Impedance Matching . . . . . . . . . . . . . . . . 108

A.3 Stineman Smoothing Algorithm from Kaleidagraph 4.1.0 . . . . . . . 109

A.4 Electromagnetic Noise Considerations . . . . . . . . . . . . . 110

A.4.1 Ground Loop Noise . . . . . . . . . . . . . . . . . . 111

A.4.2 What is a Ground Loop? . . . . . . . . . . . . . . . . 111

A.4.3 How does a Differential Amplifier Help? . . . . . . . . . . . . 113

A.4.4 Inductive Noise . . . . . . . . . . . . . . . . . . 113

A.4.5 Quantization Noise ............... 114

A.4.6 Dithering ......................... 115

A.5 QTPAS Flow Chart . . . . . . . . . . . 117

A.6 Signal Simulation: ModelingQTPAS.m . . . . . . . . . . . . . 118 
BIBLIOGRAPHY . . . . . . . . . . . . . 122

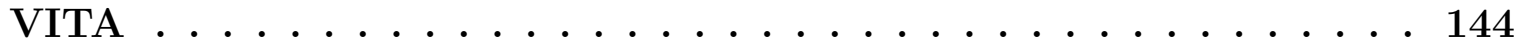




\section{LIST OF TABLES}

Table $\quad$ Page

4.1 Comparison of PAS/TIRPAS refractometry to handheld refractometry 74

4.2 Comparison of PAS/TIRPAS refractometry to existing techniques . . 77 


\section{LIST OF FIGURES}

Figure $\quad$ Page

1.1 Surface properties . . . . . . . . . . . . . . 3

1.2 Gold nanoparticles attached to fluorescent beads . . . . . . . . . 5

1.3 Electron microscopy schematic . . . . . . . . . . . . . . 6

1.4 Ellipsometry schematic . . . . . . . . . . . . . . 7

1.5 Atomic force microscopy schematic . . . . . . . . . . . 9

1.6 AFM: contact/tapping mode . . . . . . . . . . . . . . . . 10

2.1 Total internal reflection schematic . . . . . . . . . . . 15

2.2 Optical fiber demonstrating total internal reflection $\ldots \ldots \ldots$

2.3 Evanescent field and optical penetration depth . . . . . . . . 16

2.4 Total internal reflection fluorescence microscope $\ldots \ldots \ldots \ldots$

2.5 Evanescent field schematic for derivation . . . . . . . . . . 20

2.6 Basic photoacoustic effect $\ldots \ldots \ldots \ldots \ldots$

2.7 Acoustic plane wave $\ldots \ldots \ldots \ldots \ldots \ldots \ldots \ldots \ldots$

2.8 Optical penetration depth differences between PAS and TIRPAS . . . 26

2.9 Types of acoustic wave generation $\ldots \ldots \ldots \ldots \ldots \ldots$

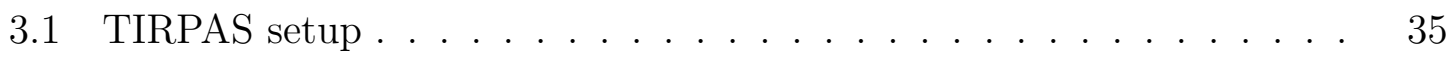


3.2 Transmission electron microscopy of $\beta$-hematin . . . . . . . . . . 38

3.3 TIRPAS critical angle determination . . . . . . . . . . . . 40

3.4 Acoustic penetration depth differences . . . . . . . . . . . . . 42

3.5 Averaged angular spectrum and outlier spectrum . . . . . . . . . 43

3.6 Angular spectra of Chlorazol black and $\beta$-hematin . . . . . . . . . . . 44

3.7 Finding $\mu_{a}$ using least squares curve fit . . . . . . . . . . . . 51

4.1 TIRPAS schematic . . . . . . . . . . . . . . 64

4.2 Working principle of TIRPAS . . . . . . . . . . . 65

4.3 Three dimensional image of experimental setup and prism holder . . . 70

4.4 Refractometry experimental setup . . . . . . . . . . . . . . . 72

4.5 PAS/TIRPAS data analysis: part I . . . . . . . . . . . . . 73

4.6 PAS/TIRPAS data analysis: part II . . . . . . . . . . . . 74

4.7 Comparing angular spectrum of dye to myoglobin . . . . . . . . . 76

5.1 Quantum tunneling photoacoustic spectroscopy schematic . . . . . 81

5.2 Optical tunneling for different film thicknesses and refractive indices . 83

5.3 Optical tunneling for a very thick film . . . . . . . . . . . . 88

5.4 QTPAS setup ........................... 90

5.5 Optical tunneling simulation . . . . . . . . . . . . . . . . . . 92

5.6 Data from substrate scan . . . . . . . . . . . . . . . . 93

5.7 Simulation from substrate scan . . . . . . . . . . . . . . 94

5.8 Scanning over time and with varying laser energy . . . . . . . . 95

6.1 Birefringence determination . . . . . . . . . . . . . . . 97

6.2 Thin film resonator . . . . . . . . . . . . . . . . . . 98 
A.1 Electrical impedance matching . . . . . . . . . . . . . . . 108

A.2 Stineman smoothing weighting distribution . . . . . . . . . . . . 110

A.3 Ground loop . . . . . . . . . . . . . . . . . . . . . 111

A.4 Angular spectrum with ground loop noise . . . . . . . . . . . . . . 112

A.5 Noise via magnetic induction . . . . . . . . . . . . . . . . . . 113

A.6 Magnetic induction noise in data . . . . . . . . . . . . . 115

A.7 Dithering to increase vertical resolution . . . . . . . . . . . 116

A.8 QTPAS experiment flow chart . . . . . . . . . . 117 


\begin{abstract}
In recent years, major research funding and commercial development has been going toward the production and characterization of increasingly useful nanomaterials. These materials such as quantum dots, nanoparticles, and thin films can increase the efficiency of solar panels, create new treatments for cancer, and vastly improve the detection capabilities for various optical sensors for biosensing. Unfortunately, to date, very few methods of characterizing these types of materials exist such as scanning electron microscopy and ellipsometry. These techniques are prohibitively expensive, cannot be used with all materials, and require rigorous preparation schemes before scanning. Therefore, a new method to characterize thin films and detect the properties of nanomaterials is needed. This study proposes a newly revived method, Total Internal Reflection Photoacoustic Spectroscopy, along with related techniques, to deliver cost effective characterization and detection for nanomaterials and thin films.
\end{abstract}




\section{Chapter 1}

\section{Introduction}

Over the course of human history there have been many technological revolutions, from the industrial revolution in the late 1700's to the information age that occurred after the early 1970's. In the 21st century, humanity has encountered another significant revolution with our discovery of the utility of nanomaterials. The discovery of materials below 100 nanometers in size with unbelievably useful properties, like superhydrophobicity, fluorescence, and strength and surface area hundreds of times higher than steel per gram, has led to the re-imagination of our medical devices, computers, and increasingly more scarce building materials. Current household products like Rust-oleum's Neverwet, which transforms any common surface into a superhydrophobic surface, stands to change every surface we touch by creating extremely simple methods of cleaning everything from clothing to cars, and stand to lessen our world's disease propagation by creating an impenetrable barrier to growing bacteria.

All of these technological marvels would not have been possible without a firm understanding of the underlying quantum, mechanical, electrical, optical, and thermal 
properties of these classically strange materials. To understand these materials we must look at properties of the surface as shown in Figure 1.1 as opposed to the bulk material properties of the interior molecules and atoms. Our capabilities to probe these surface properties on the nano level are dependent upon a handful of technologies such as electron microscopy, ellipsometry, and atomic force microscopy. This is due to the fact that below 250 nanometers, traditional microscopy methods cannot resolve structures due to the optical diffraction limit. These exotic methods share the commonality that they can resolve structures below 200 nanometers, thus making them super resolution instruments, however, their ability to discern basic properties beyond imaging is extremely limited. Therefore, new methods to characterize materials in the UV/VIS/NIR region of the optical spectrum are needed to test these new nanomaterials at understandable conditions more akin to real life situations.

\subsection{Mesoporous Materials}

One class of materials that has generated a significant amount of interest for material characterization is mesoporous materials. These materials have nano sized pores from approximately 2-50 $\mathrm{nm}$ in size, which can be used for a variety of purposes, most obviously as a filter or catalyst support [1]. In addition to those traditional properties, mesoporous materials are being increasingly used in biomedical related fields like diagnostic optics [2,3], military applications such as antireflection coatings

[4], gas sensors [5], and even as tri-modal imaging nano particles [6], which stand to revolutionize medical biosensing for cancer and other diseases. 


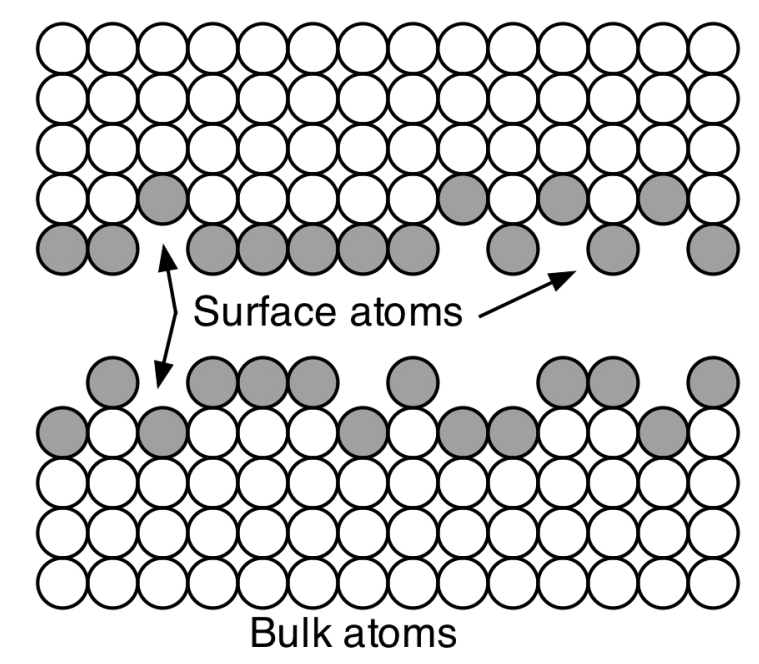

Figure 1.1: Surfaces control the overall properties of materials.

\subsection{Current Techniques}

There are effectively three main techniques to help us characterize nanomaterials; electron microscopy, ellipsometry, and atomic force microscopy. Electron microscopy, invented in the early 1930 's, gave the scientific community the ability to break the optical diffraction limit and look at structures below 200 nanometers. Traditional microscopy is limited by the optical diffraction limit given by

$$
d=\frac{\lambda}{(2 n \sin \theta)},
$$

where $d$ is the diameter of a diffraction limited spot, $n$ is the refractive index of the medium being imaged, and $\theta$ is the half angle of the maximum cone of light that can enter the lens. However, due to the work done by deBroglie in the 1920's, it was recognized that the effective diffraction limit of non-light based particles such as electrons would be much smaller than that of a comparable visible wavelength of 
light. Green light has a wavelength of approximately $532 \mathrm{~nm}$ that gives a theoretical optical diffraction limit in air of 190 nanometers. However, the deBroglie equation, shown in (1.1), calculates an electron's wavelength of approximately 5.4 picometers from (1.2) (assuming $100 \mathrm{kV}$ microscope voltage). Thus, the diffraction limit of the electron microscope is approximately 0.24 nanometers given by

$$
\lambda=h / p
$$

and

$$
\lambda=\frac{h}{2 m e V},
$$

where $\lambda$ is the wavelength of the particle, $h$ is Planck's constant, $p$ is the momentum, $m$ is the electron's rest mass, $e$ is the electronic charge, and $V$ is the accelerating voltage of the electron microscope. Therefore, using an electron microscope allows for an approximately 1000 fold increase in resolving power when compared to a traditional light microscope. Various modes of the electron microscope can be used for a variety of purposes suited to the sample and testing parameters needed for evaluation.

\subsubsection{Transmission Electron Microscope}

Electron microscopes work on the principle of interacting electron beams with the sample in much the same way that a traditional microscope exposes samples to light. In transmission mode, the electron microscope passes electrons through a micronthin sectioned material of interest. As the electrons pass through the material image contrast is formed by the absorption and scattering of electrons as they encounter the 
material. In transmission mode, electron microscopes, while extremely valuable for scientific research labs, do have several drawbacks that prevent their widespread use in industrial applications today. Although the resolution of TEMs is higher than that of scanning electron microscopes (SEM), the sample thickness is generally limited to micron-thin materials. Additionally, TEMs are very expensive $(\$ 2,000,000)$, tend to be room-sized instruments, and require constant maintenance.

\subsubsection{Scanning Electron Microscope}
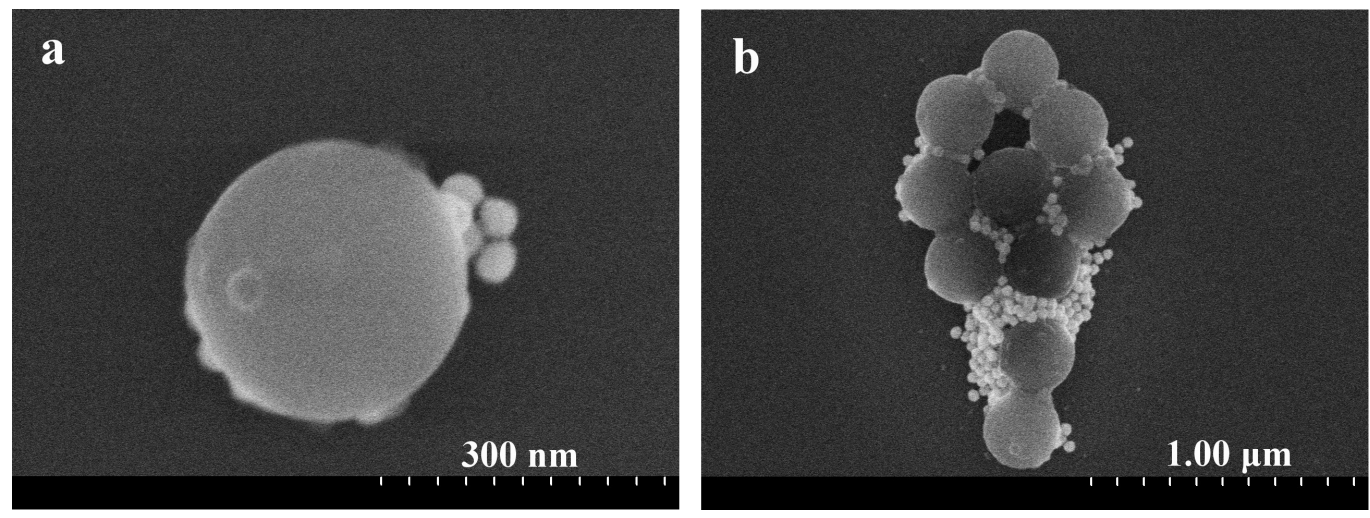

Figure 1.2: Gold nanoparticles attached to fluorescent beads have been used for their high optical absorption properties for the early detection of breast cancer. [7]

Electron microscopes can be used in the scanning mode that allows for physically larger samples to be used for scanning purposes. In contrast to transmission mode, scanning electron microscopes use secondary electrons for image contrast. Secondary electrons are electrons that have been removed from the material of interest by the interaction of the SEM's high speed electrons with the sample material. These secondary electrons generally have a mean free path on the order of a few nanometers and therefore the imaging of a material can only lightly penetrate the surface and 
is therefore used primarily for imaging material surfaces. Metal nanoparticles, as shown in Figure 1.2, show up particularly well in electron microscopes due to their conductive properties. A schematic of a typical SEM is shown in Figure 1.3.

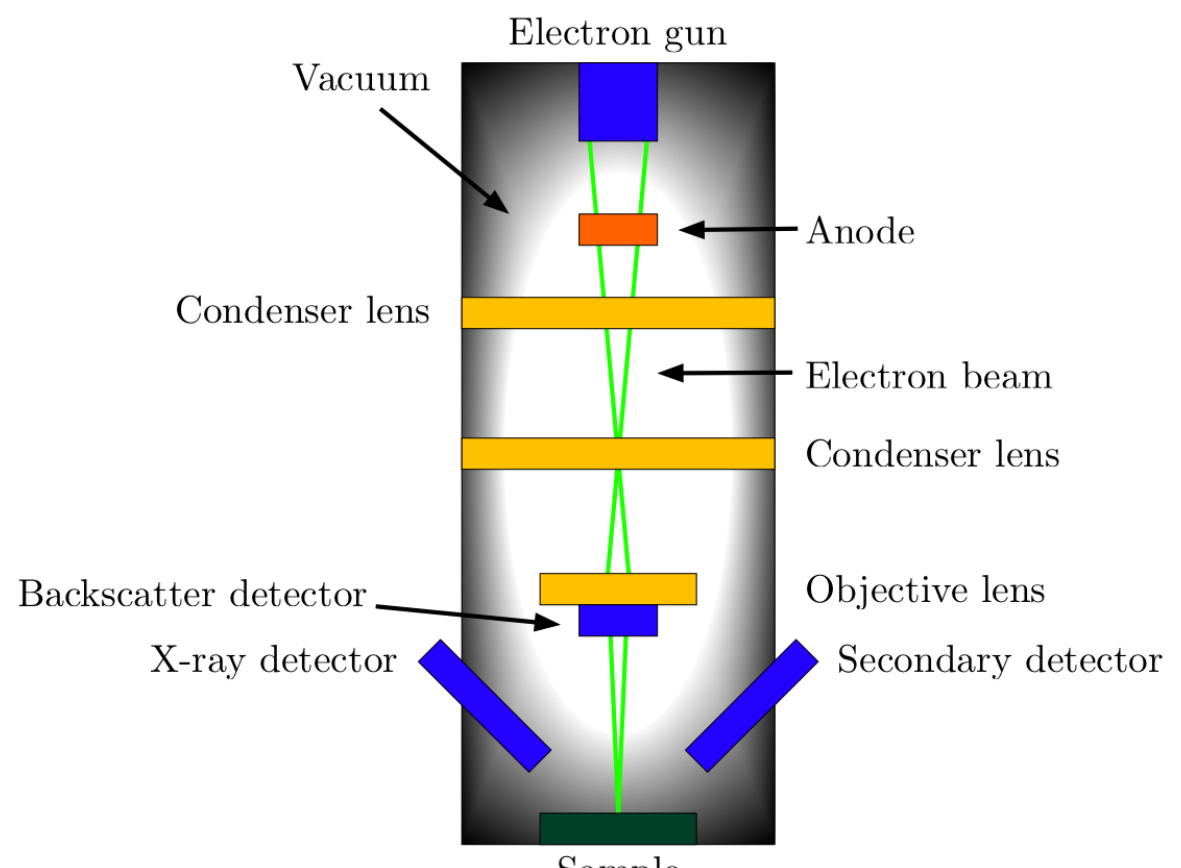

Sample

Figure 1.3: Electron microscopy is a complex series of condenser lenses similar to optical microscopy.

Unfortunately, SEM's tend to have drawbacks in terms of cost $(\$ 100,000)$, intense sample preparation (most samples have to be coated in some type of metal), and most samples require a vacuum environment. However, there are some SEM models that have the ability to look at wet samples in a limited way. 


\subsubsection{Ellipsometry}

Ellipsometry is an optical technique that measures changes in the linear polarization of an incident light beam reflected from sample surfaces in order to determine the optical properties of the material such as thickness and refractive index. As shown in Figure 1.4, a laser beam of known polarization is reflected off the surface of a sample. The reflection is then measured to determine the amount the polarized axis shifted after interacting with the sample.

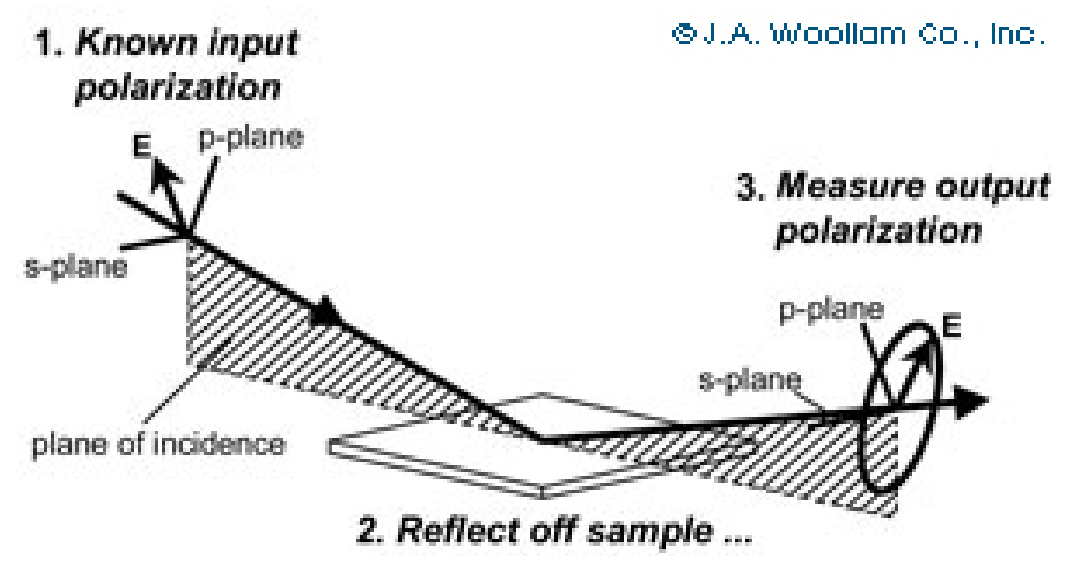

Figure 1.4: Light interacts with a sample in ellipsometry via polarization changes from interacting with the surface layers. (Reproduced with permission from J.A. Woollam at http://www . jawoollam.com/tutorial_4.html)

Ellipsometry can be described by

$$
\langle\tilde{\varepsilon}\rangle=\sin ^{2} \phi\left[1+\left(\frac{1-\rho}{1+\rho}\right)^{2} \tan ^{2} \phi\right],
$$

where $\langle\tilde{\varepsilon}\rangle$ is the electromagnetic permittivity, $\phi$ is the angle of incidence of the beam relative to the sample, and $\rho=\sqrt{R_{p} / R_{s}}$, where $R_{p} / R_{s}$ represents the reflected values for the $P$ polarization and $S$ polarization respectively. 
Ellipsometry has the ability to measure many different types of properties, from thickness and refractive index to composition, crystallinity, surface roughness, and doping concentration. Unfortunately, ellipsometry suffers from several drawbacks. Most notably, the films that can be analyzed must be relatively non-pigmented materials in order to reflect back sufficient light for the ellipsometer to function. In addition, the surfaces of the films being analyzed must be polished before analysis in order to provide a high enough reflection to be analyzed. Finally, a complex Cauchy model must be used to fit the coefficients in the equation to find thickness and refractive index that requires extensive training on the user's side for appropriate ellipsometer functionality.

\subsubsection{Atomic Force Microscopy}

Atomic force microscopy as shown in Figure 1.5 is the final existing technology that can be used to evaluate surfaces and nanomaterials. Atomic Force Microscopy, invented in 1986 by Binnig, Quate, and Gerber, is a piezoelectric system that can probe the surface of a material by interacting the probe with the surface and recording the corresponding movement over the surface. The working principle of this device is that a very sharp probe is slid across the surface of the sample and small deflections of that probe are measured that can be used to create a topographical map or three dimensional image of the surface. There are three modes that an AFM is generally used in that can determine different properties about the surface of a material. 


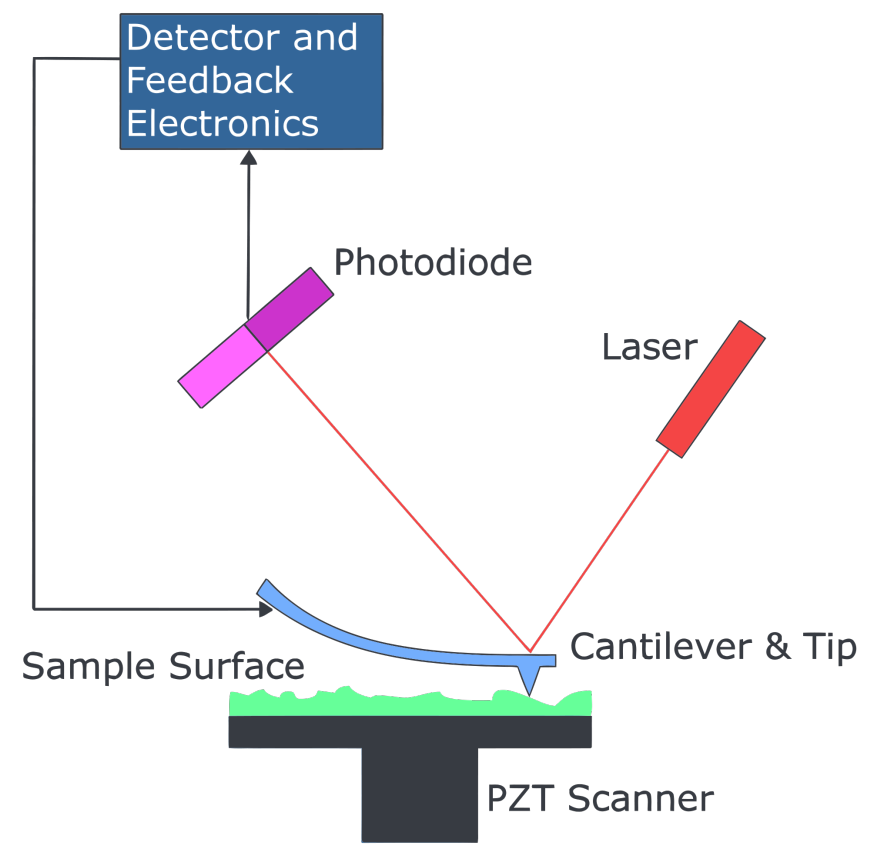

Figure 1.5: Atomic Force Microscopy driven by a piezo scanner can scan very small changes in surface roughness and can image materials at the nano level.

\section{Contact Mode}

In contact mode [8], the Atomic Force Microscope uses its AFM probe along with feedback from the piezoelectric element to keep the probe constantly in contact with the sample. In this way the AFM probe can follow the contours of the surface and respond to differences in height that translate to differences in piezoelectric voltage to recreate an image. This process is shown in Figure 1.6.

Contact mode is generally used to obtain the highest resolution imaging from the AFM to image atomically smooth materials like silicon and glass wafers. Unfortunately, contact mode suffers from the drawback that since it is in contact with the sample, some sample damage and image distortion may occur due to the contact of 
the AFM tip with the sample. Additionally, as with all AFM techniques, imaging takes a very long time due to the device having to raster scan the film of interest over several minutes.

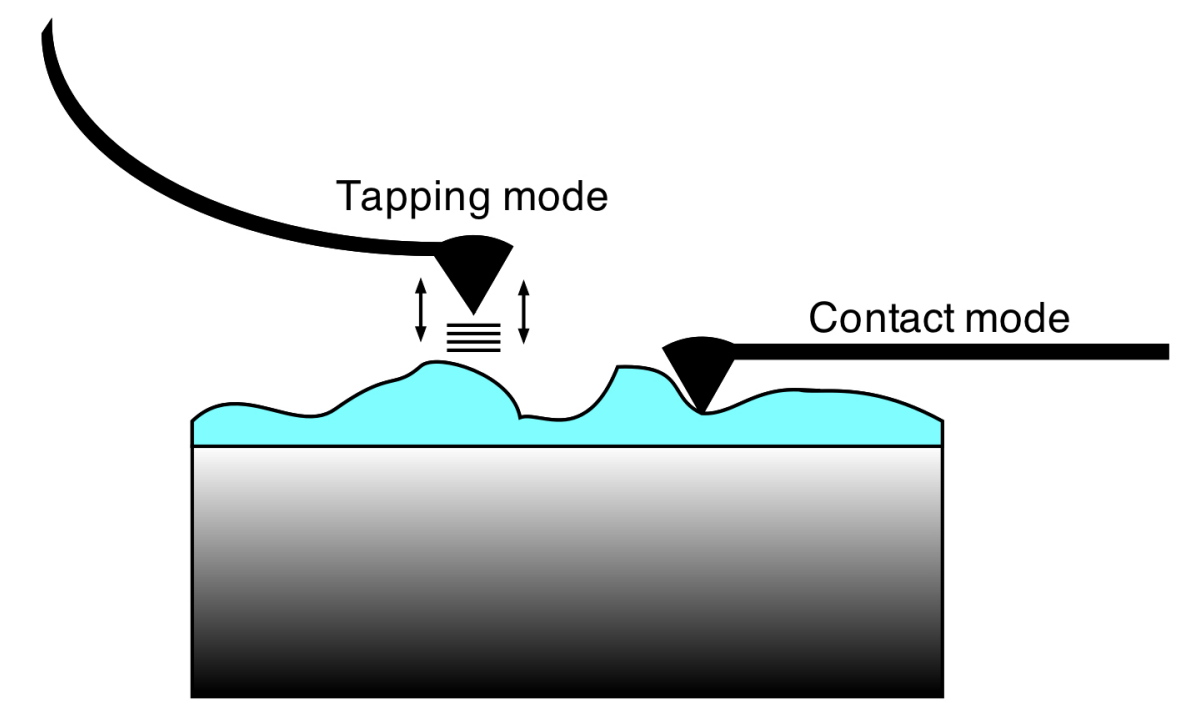

Figure 1.6: Several modes of atomic force microscopy exist such as contact and tapping modes.

\section{Non-Contact Mode}

In contrast to contact mode, non-contact mode [9] uses the AFM probe tip at 5-15 $\mathrm{nm}$ away from the surface of the sample for scanning. Van der waals forces resulting from the proximity of the probe to the sample allow for topographical changes to be measured and thus an image to be formed by the device. Non-contact mode prevents the issues apparent with contact mode such as sample damage, tip damage, and image distortion. However, due to the fact that Van der waals forces tend to be much smaller than the forces observed in contact mode, only relatively large changes can be seen in the three-dimensional image and therefore the technique has less resolution 
than contact mode.

\section{Tapping Mode}

In tapping mode AFM [10], the strengths of both contact mode and non-contact mode are combined by modulating the contact of the probe tip with the sample. The probe is modulated by the piezoelectric element to vibrate at the probe's natural resonant frequency. At this frequency the probe stays in contact with the sample for

a moment, then the probe is moved away from the sample out of the range of the contact forces. This process is repeated during the entire length of the scan. Since the probe tip is not in constant contact with the surface, no surface damage occurs, and since the probe is in contact with the surface for a short amount of time, high resolution images can be obtained with ease. Unfortunately, even this combined AFM method suffers from slow scan speed, and limited imaging area $(150 \mu \mathrm{m}$ x $150 \mu \mathrm{m})$

\subsection{The Photoacoustic Effect}

In 1880, with the invention of Alexander Graham Bell's photophone, the photoacoustic effect, or light induced sound, was created. The photoacoustic effect is the optical absorption of pulsed light that is converted into pressure waves, or sound. This effect was nearly forgotten by the scientific community until the early 1970s [11] with its rediscovery due to advancement in laser and microphone technology. Since then, the photoacoustic effect has been used in almost every field to fill niche applications from biomedical imaging to thin film analysis to non-destructive testing of armor in military vehicles. 


\subsubsection{Biomedical Applications of the Photoacoustic Effect}

In the biomedical arena, there are effectively two main areas of application of the photoacoustic effect. One area of study has been cancer detection by Viator $[7,12-27]$ and others [28]. Other types of cancers beyond metastatic melanoma have also been detected using this technique. Even the possibility of detecting semen [29] for fertility studies been investigated using this technique. Interestingly enough, two methods of circulating tumor cell detection have been used over the past few years, an in vitro method [15] and an in vivo method [30] have been used for circulating tumor cell detection.

The second area of application of the photoacoustic effect is photoacoustic imaging and optical property evaluation. Many papers have been published on photoacoustic imaging [25,31-47] and general optical evaulation [48-53]. However, several applications of photoacoustic imaging are currently being pursued such as burn depth imaging $[32,40,52]$ and transcranial brain imaging [54]. Finally, even methods that use pulsed lasers that do not directly desire the photoacoustic effect can still affect measurements [55] through added effects for the permeabilization of cells.

\subsection{Total Internal Reflection Photoacoustic Spec- troscopy}

A recently rediscovered technology, Total Internal Reflection Photoacoustic Spectroscopy, combines several different technologies together to form a device that has great capability for determining quantum, mechanical, electrical, optical, and thermal properties of materials at the nano scale. TIRPAS functions by gathering acoustic 
waves that have been generated by the optical absorption of energy through the interaction of the sample with evanescently coupled photons through a prism/sample interface. TIRPAS has many advantages over traditional technologies such as limited cost, limited sample preparation, and optical spectroscopic evaluation. The overall working principle and functionality of TIRPAS is explained in Chapter 2. 


\section{Chapter 2}

\section{TIRPAS and SPRPAS}

\subsection{Total Internal Reflection}

In order to understand Total Internal Reflection Photoacoustic Spectroscopy we must first explain the definition of total internal reflection. Total internal reflection refers to a state of light where an incident beam impinges upon an interface between two materials $n_{1}$ and $n_{2}$ such that $100 \%$ of the incident light is reflected away from the interface as shown in Figure 2.1. The condition needed for this reflection to occur comes from Snell's law given by

$$
n_{1} \sin \theta_{1}=n_{2} \sin \theta_{2},
$$

where the refracted portion of the light $\left(\theta_{2}\right.$ goes to 90 degrees so $\left.\sin \theta_{2}=1\right)$ disappears and the light is totally internally reflected away from the interface, which is described by 


$$
\theta_{1}=\theta_{\text {critical }}=\sin ^{-1}\left[\frac{n_{2}}{n_{1}}\right] .
$$

Total internal reflection is the effect that allows fiber optic waveguides to guide light large distances $(\mathrm{km})$ for computational and telecommunication applications as in Figure 2.2. Additionally, total internal reflection is used in a variety of imaging technologies, most notably, the total internal reflection microscope as shown in Figure 2.4 that uses the interaction of the evanescent field with a fluorophore to see below the optical diffraction limit.

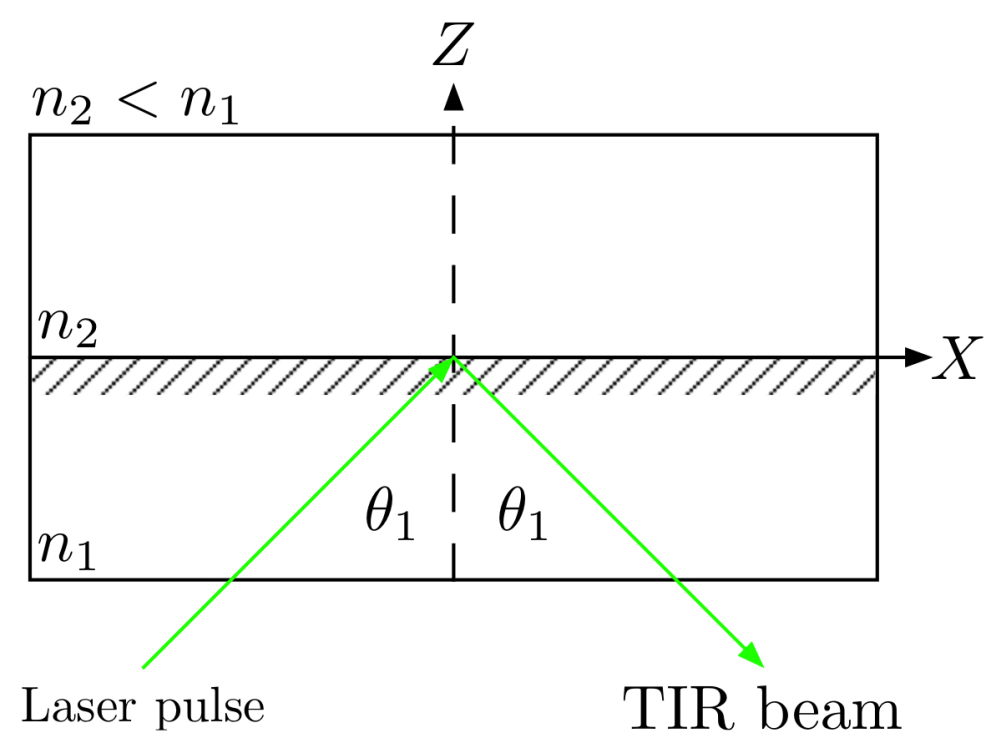

Figure 2.1: Total internal reflection at an interface can be described by this schematic.

Imaging below the wavelength of light is achieved with high contrast due to the confinement of the evanescent field to the interface between the sample and the prism. Beyond simple imaging, spectroscopic analysis and even dual modality microscopes have been created to utilize total internal reflection to image nanomaterials in a cost effective manner. 


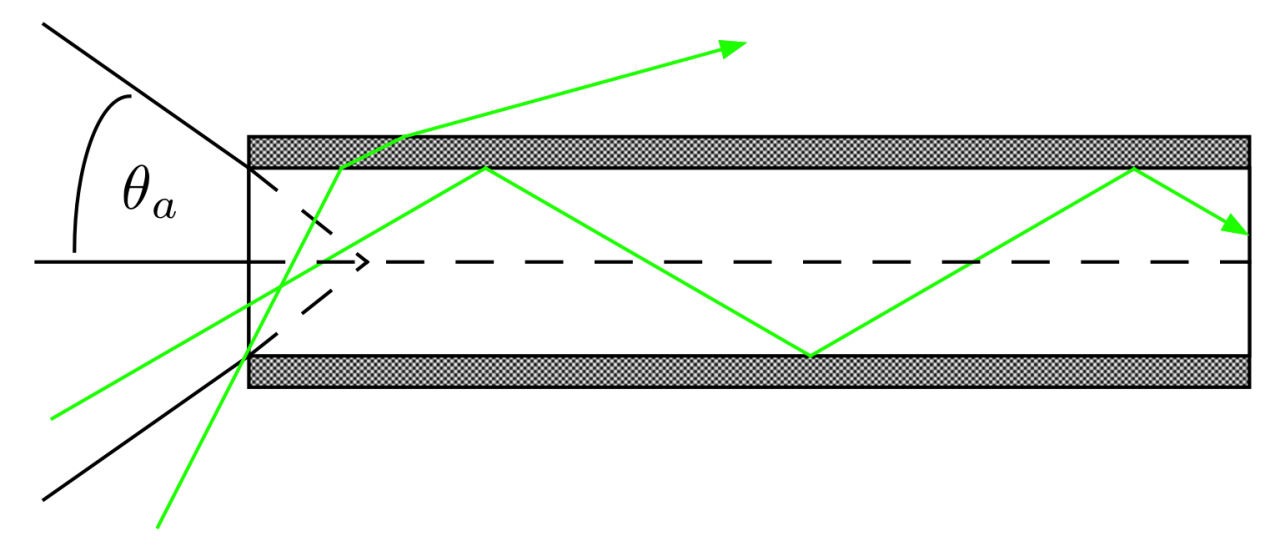

Figure 2.2: Optical fibers can only accept light within a given acceptance angle.

\subsubsection{The Evanescent Field}

The evanescent field is a non-propagating optical field [56] that is generated under the condition that a beam of light strikes an interface where the incoming medium has a higher refractive index than the outgoing medium and the light is striking at an angle larger than the critical angle as shown in Figure 2.3. This non-propagating field can be derived by starting with a few assumptions.

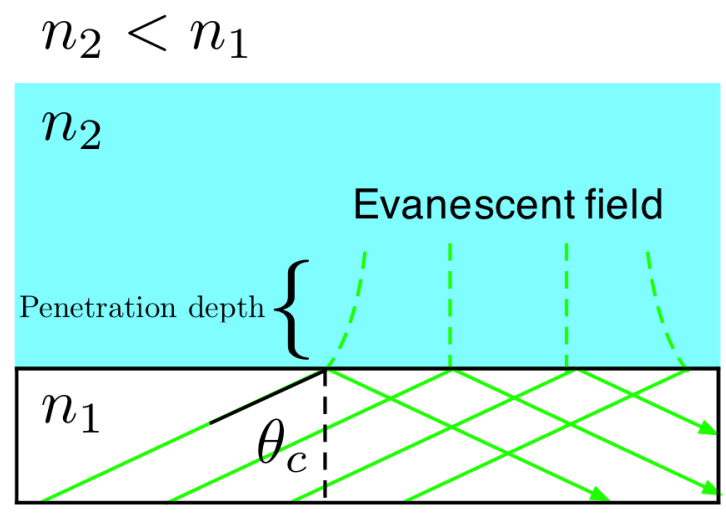

Figure 2.3: The evanescent field has a distinct optical penetration depth based upon factors such as excitation angle and material properties. 


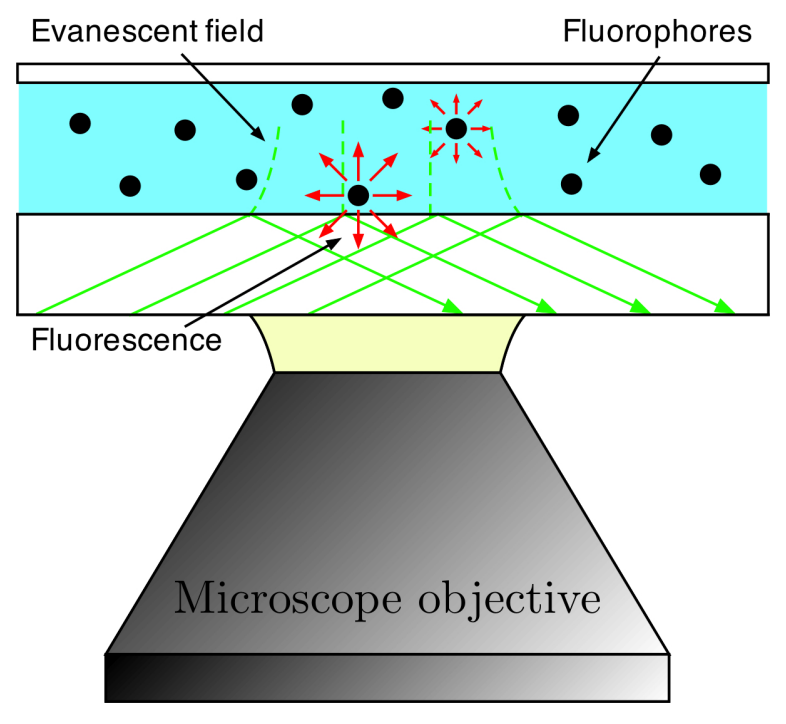

Figure 2.4: Total internal reflection fluorescence microscopy can examine materials on the nanometer level such as fluorescent dyes.

\section{Deriving the Existence of the Evanescent Field}

Maxwell's equations [57] are given by

$$
\begin{gathered}
\nabla \cdot E=0, \\
\nabla \times E=\frac{-\partial B}{\partial t}, \\
\nabla \cdot B=0,
\end{gathered}
$$

and

$$
\nabla \times B=\mu_{0} \epsilon_{0} \frac{\partial E}{\partial t} .
$$


Since the evanescent field should be described from the perspective of the electric field I will use (2.2) and (2.3) to start the derivation. To simplify (2.3) it is necessary to take the curl that gives

$$
\nabla \times(\nabla \times E)=\frac{-\partial}{\partial t} \nabla \times B
$$

Substituting (2.1.1) into (2.4) gives

$$
\nabla \times(\nabla \times E)=\frac{-\partial}{\partial t} \mu_{0} \epsilon_{0} \frac{\partial E}{\partial t}
$$

Equation (2.5) can be simplified by using the curl of the curl identity given by $\nabla \times$ $(\nabla \times E)=\nabla(\nabla \cdot E)-\nabla^{2} E$ that gives

$$
\nabla(\nabla \cdot E)-\nabla^{2} E=\frac{-\partial}{\partial t} \mu_{0} \epsilon_{0} \frac{\partial E}{\partial t}
$$

Equation (2.6) can be simplified further using (2.2) that cancels a term to give

$$
-\nabla^{2} E=\mu_{0} \epsilon_{0} \frac{-\partial^{2} E}{\partial t}
$$

The definition of the speed of light in a vacuum is $c=\frac{1}{\sqrt{\mu_{0} \epsilon_{0}}}$ so $(2.7)$ can be further simplified to 


$$
\frac{\partial^{2} E}{\partial t}-c_{0}^{2} \nabla^{2} E=0
$$

Equation (2.8) can be simplified still to (2.9), which is the electromagnetic wave equation given by

$$
\frac{\partial^{2} E}{\partial t}-c_{0}^{2} \frac{\partial^{2} E}{\partial \vec{r}^{2}}=0
$$

where $\vec{r}$ is a position vector. A solution to (2.9) is given by

$$
E(x, t)=E_{0} \cos (\vec{k} \cdot \vec{r}-\omega t)
$$

where $\vec{k}$ is a propagation vector. By using phasor notation it can be assumed that $\cos (\vec{k} \cdot \vec{r}-\omega t)=\Re\left\{e^{i(\vec{k} \cdot \vec{r}-\omega t)}\right\}$ and therefore we can rewrite $(2.10)$ to get

$$
E(x, t)=E_{0} e^{i(\vec{k} \cdot \vec{r}-\omega t)}
$$

which is the equation to describe a plane polarized beam of light that is incident upon an interface as shown in Figure 2.5. The problem is set up as an interface between two dielectric media $n_{1}$ and $n_{2}$ such that $n_{1}>n_{2}$ as shown in Figure 2.5, which can be described by a modified version of (2.11) given by

$$
E_{2}=E_{02} e^{-i\left(\omega t-\vec{k}_{2} \cdot \vec{r}\right)}
$$




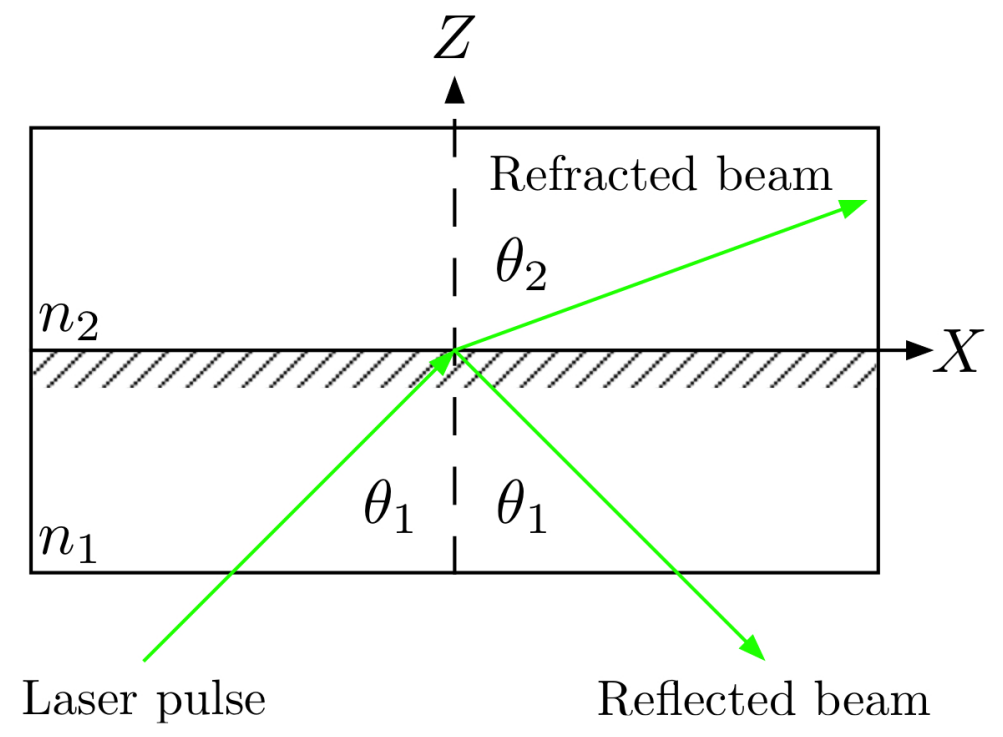

Figure 2.5: This is a schematic representation of reflection and refraction to aid with evanescent field derivation.

The propagation vector in the second medium is given by

$$
\vec{k}_{2}=\left(k_{2} \sin \theta_{2}, 0, k_{2} \cos \theta_{2}\right)
$$

The dot product of the propagation number, $\vec{k}_{2}$, and the position vector $\vec{r}$ is given by

$$
\vec{r} \cdot \vec{k}_{2}=k_{1} x \sin \theta_{2}+k_{2} z \cos \theta_{2}
$$

where $x$ and $z$ are position. Equation (2.13) can be substituted into (2.12), which gives

$$
E_{2}=E_{02} e^{-i\left(\omega t-k_{2} x \sin \theta_{2}-k_{2} z \cos \theta_{2}\right)}
$$

After applying the Pythagorean trigonometric identity we have 


$$
E_{2}=E_{02} e^{-i\left(\omega t-k_{2} x \sin \theta_{2} \pm k_{2} z\left(1-\sin ^{2} \theta_{2}\right)^{\frac{1}{2}}\right)} .
$$

Equation (2.1), or Snell's law, can then be applied to get rid of the $\theta_{2}$ so that the equation can be defined entirely by the incident angle, $\theta_{1}$, given as

$$
E_{2}=E_{02} e^{-i\left(\omega t-k_{2} x \frac{n_{1} \sin \theta_{1}}{n_{2}} \pm k_{2} z\left(1-\left(\frac{n_{1} \sin \theta_{1}}{n_{2}}\right)^{2}\right)^{\frac{1}{2}}\right)}
$$

where $n_{1}$ and $n_{2}$ are the refractive indices of the two materials. If we distribute the negative sign we get

$$
E_{2}=E_{02} e^{i\left(-\omega t+k_{2} x \frac{n_{1} \sin \theta_{1}}{n_{2}} \pm k_{2} z\left(1-\left(\frac{n_{1} \sin \theta_{1}}{n_{2}}\right)^{2}\right)^{\frac{1}{2}}\right)}
$$

where the propagation number, $k_{2}$ can be defined as $k_{2}=\frac{\lambda}{2 \pi}$. In addition, the wavelength can be adjusted for different media refractive indices by using $\lambda=\frac{\lambda_{0}}{n_{t}}$ where $\lambda_{0}$ is the wavelength in a vacuum. Additionally, since $\sqrt{1-a}=i \sqrt{a-1}$ the exponential function in (2.14) can be split up into its constituent $\mathrm{x}$ and $\mathrm{z}$ components that allows the imaginary component to be removed to get

$$
E_{2}=E_{02} e^{i\left(-\omega t+\frac{2 \pi n_{2}}{\lambda_{0}} x \frac{n_{1} \sin \theta_{1}}{n_{2}}\right)} e^{\left( \pm \frac{2 \pi n_{2}}{\lambda_{0}} z\left(\left(\frac{n_{1} \sin \theta_{1}}{n_{2}}\right)^{2}-1\right)^{\frac{1}{2}}\right)} .
$$

After simplification we obtain

$$
E_{2}=E_{02} e^{i\left(-\omega t+\frac{2 \pi n_{2}}{\lambda_{0}} x \frac{n_{1} \sin \theta_{1}}{n_{2}}\right)} e^{\left( \pm \frac{2 \pi n_{1}}{\lambda_{0}} z\left(\sin \theta_{1}^{2}-\left(\frac{n_{2}}{n_{1}}\right)^{2}\right)^{\frac{1}{2}}\right)} .
$$

Finally, we can define (2.15) in terms of $z$ and, $\delta$, the penetration depth of the 
evanescent field given by

$$
E_{2}=E_{02} e^{i\left(-\omega t+\frac{2 \pi n_{2}}{\lambda_{0}} x \frac{n_{1} \sin \theta_{1}}{n_{2}}\right)} e^{\frac{-z}{\delta}}
$$

where $\delta=\frac{\lambda_{0}}{2 \pi n_{1}\left(\sin \theta_{1}^{2}-\left(\frac{n_{2}}{n_{1}}\right)^{2}\right)^{\frac{1}{2}}}$ and is a measure of the penetration depth of the electric field component of the evanescent field into the rarer medium. Since the electric field of the evanescent field is typically not measured during an experiment it is useful to form the equation in terms of incident number of photons or $I$ and $I_{0}$. To convert from the electric field to intensity we use

$$
I=\epsilon_{0} c E_{2}^{2}
$$

where $\mathrm{I}$ is the light intensity, $\epsilon_{0}$ is the permittivity of free space, and $\mathrm{c}$ is the speed of light. Applying (2.17) to (2.16) gives the evanescent field's penetration depth in terms of light intensity given by

$$
I=I_{0} e^{\frac{-2 z}{\delta}}
$$

where $I_{0}=\epsilon_{0} c E_{02}^{2} e^{2 i\left(-\omega t+\frac{2 \pi n_{2}}{\lambda_{0}} x \frac{n_{1} \sin \theta_{1}}{n_{2}}\right)}$. The factor of 2 in the exponentials is introduced due to the $E_{2}^{2}$ term in (2.17).

\subsubsection{Photoacoustic Effect}

The photoacoustic effect [58], is the generation of thermoelastic pressure waves via the localized optical absorption of light into a material. The photoacoustic effect was discovered in the early 1880's by Alexander Graham Bell as he was trying to invent 
wireless communication in the form of the photophone. In essence the photoacoustic effect can be described by Figure 2.6.

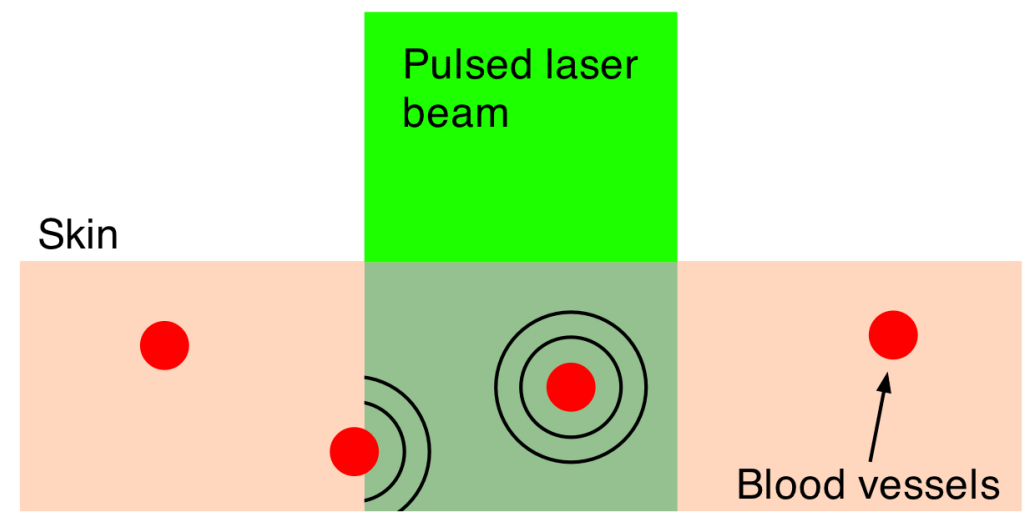

Figure 2.6: Laser excitation to generate the photoacoustic effect in tissue has many uses including imaging blood vessels and cancerous tumors.

The photoacoustic effect in this case is a pulsed beam of concentrated laser light that can be absorbed by an object imbedded deep within tissue. The equation that describes the photoacoustic effect, surprisingly enough, is the same equation that describes the evanescent field, known as the wave equation. In the experiments conducted for this dissertation, all acoustic sources were assumed to be plane wave sources (that simplifies the mathematical description). The assumption of plane waves is valid due to the fact that plane waves are observed whenever the optical penetration depth of the incident light is less than $1 / 10$ the size of the diameter of the incident laser beam. Since only $\sim 500 \mathrm{~nm}$ of optical penetration depth is possible using TIRPAS and related techniques, this assumption is valid since the size of the beam was at least $1 \mathrm{~mm}$ in diameter. Therefore, the photoacoustic effect in one dimension, $x$, can be described by (2.18) that is very similar to the general wave equation describing the evanescent field in (2.9). 


$$
\frac{\partial^{2} p}{\partial x^{2}}-\frac{1}{c^{2}} \frac{\partial^{2} p}{\partial t^{2}}=0
$$

Equation (2.18) describes a 1 dimensional acoustic wave with pressure that varies in both position and time. The solution of (2.18) is given by

$$
p(x, t)=f(x-c t)+f(x+c t) .
$$

In terms stress confined photoacoustic excitation, we obtain a final equation describing the plane waves as

$$
p(x, t)=\frac{1}{2} \Gamma \mu_{a} H_{0} e^{-\mu_{a}(x-c t)}+\frac{1}{2} \Gamma \mu_{a} H_{0} e^{-\mu_{a}(x+c t)},
$$

where $p$ is pressure, $\Gamma$ is the Grünesian coefficient, $\mu_{a}$ is the absorption coefficient, $H_{0}$ is the radiant exposure of the laser beam, $c$ is the speed of sound in the excited medium, $x$ is length, and $t$ is time. Figure 2.7 shows the transducer signal corresponding to a detected plane wave.

Interestingly, the shape of the acoustic wave follows the photonic distribution within the material of interest for all plane wave excitation. Since photonic distribution into a material of interest follows Beer's law shown in (2.20), it is possible to apply a non-linear least squares curve fitting algorithm to a measured acoustic wave (Figure 2.7) and fit to the upward stroke of the acoustic wave to get an indication of the value of $\mu_{a}$, or absorption coefficient, to estimate the concentration of the absorber via (2.20) and (2.21). Of course, without a measurement of the error with a single non-linear least squares fit this method is used in an exploratory manner to get a general idea of the optical absorption of a material rather than the exact value. 


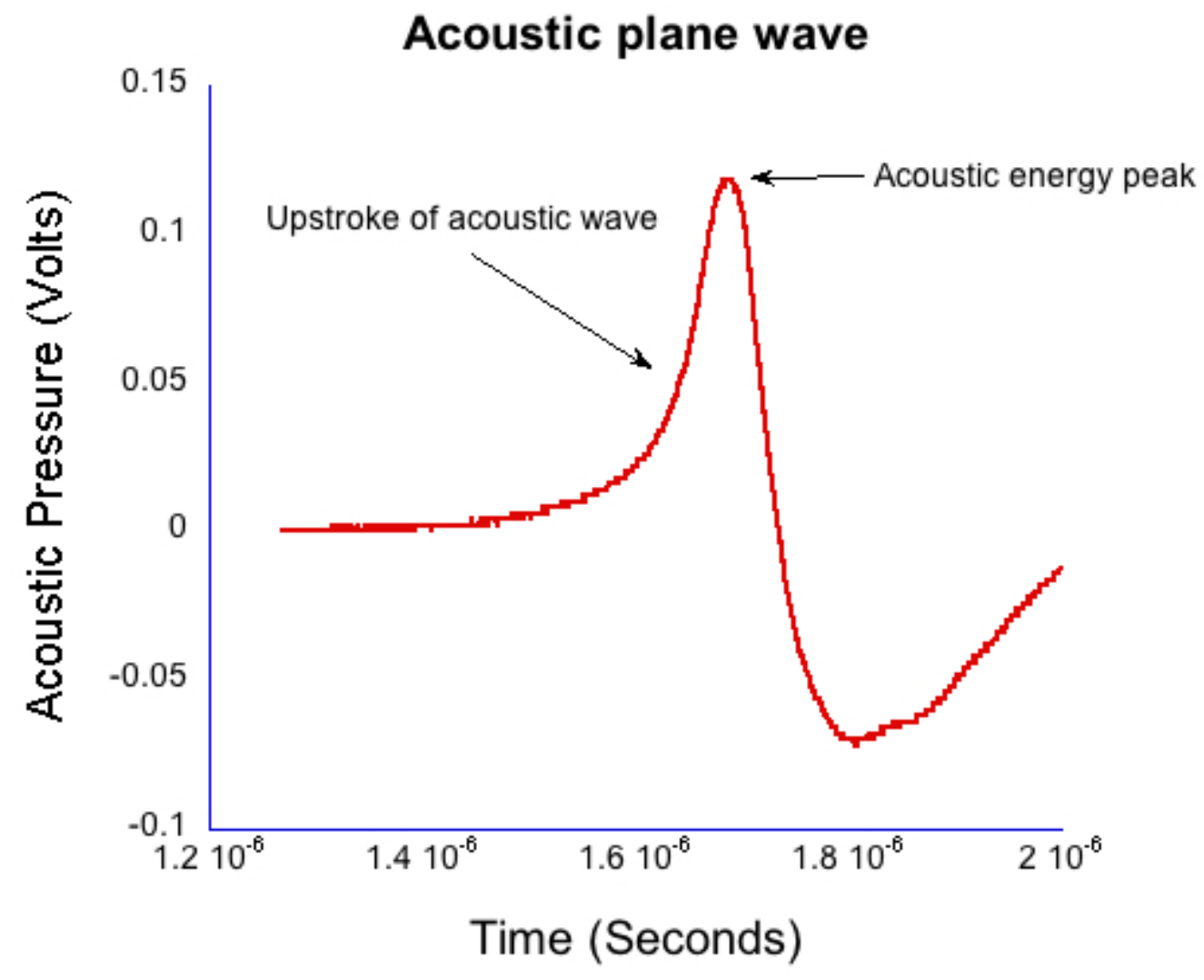

Figure 2.7: This electrical signal is an example of an acoustic plane wave generated from photoacoustic excitation.

The experimental details to obtain Figure 2.7 can be found in [59].

$$
\begin{gathered}
I=I_{0} e^{-\mu_{a} x} \\
\mu_{a}=2.303 \epsilon c
\end{gathered}
$$




\subsection{Total Internal Reflection Photoacoustic Spec- troscopy}

Total Internal Reflection Photoacoustic Spectroscopy, or TIRPAS, is the combination of both the total internal reflection effect and the photoacoustic effects together to form a new device. Specifically, the physical excitation achieved by TIRPAS can be described by Figure 2.8.
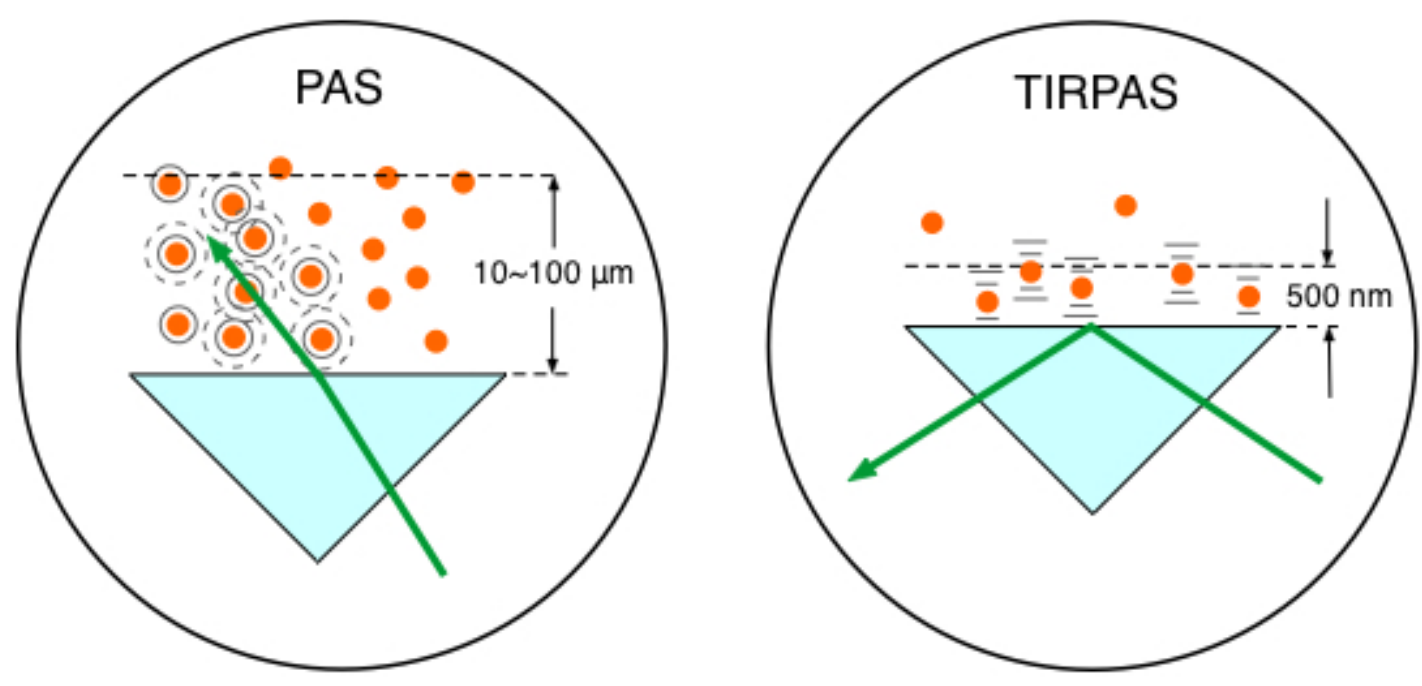

Figure 2.8: Optical penetration depth differences between PAS and TIRPAS are at least an order of magnitude different.

As can be seen from Figure 2.8, the excitation of the photoacoustic effect by absorption of light normal to an interface will excite the chromophores following the photonic distribution described by the Beer-Lambert law, or (2.20), that represents a penetration depth of 10-100 $\mu \mathrm{m}$. TIRPAS, on the contrary, only excites chromophores that are within the evanescent field that is typically on the order of the wavelength $(500 \mathrm{~nm})$. This constrained optical excitation by the evanescent field gives rise to some interesting properties of TIRPAS induced acoustic waves. Most notably, since 
the optical penetration depth is on the order of $500 \mathrm{~nm}$ regardless of the absorption of the material of interest, under most circumstances, we can treat the acoustic wave generation as nearly ideal plane waves. This is due to the fact that the spot size is usually at least 1000 times larger in diameter than the optical penetration depth of the evanescent field. This configuration, as shown in Figure 2.9, causes the acoustic energy to propagate primarily in the forward and backward directions causing a bipolar waveform as shown in Figure 2.7.
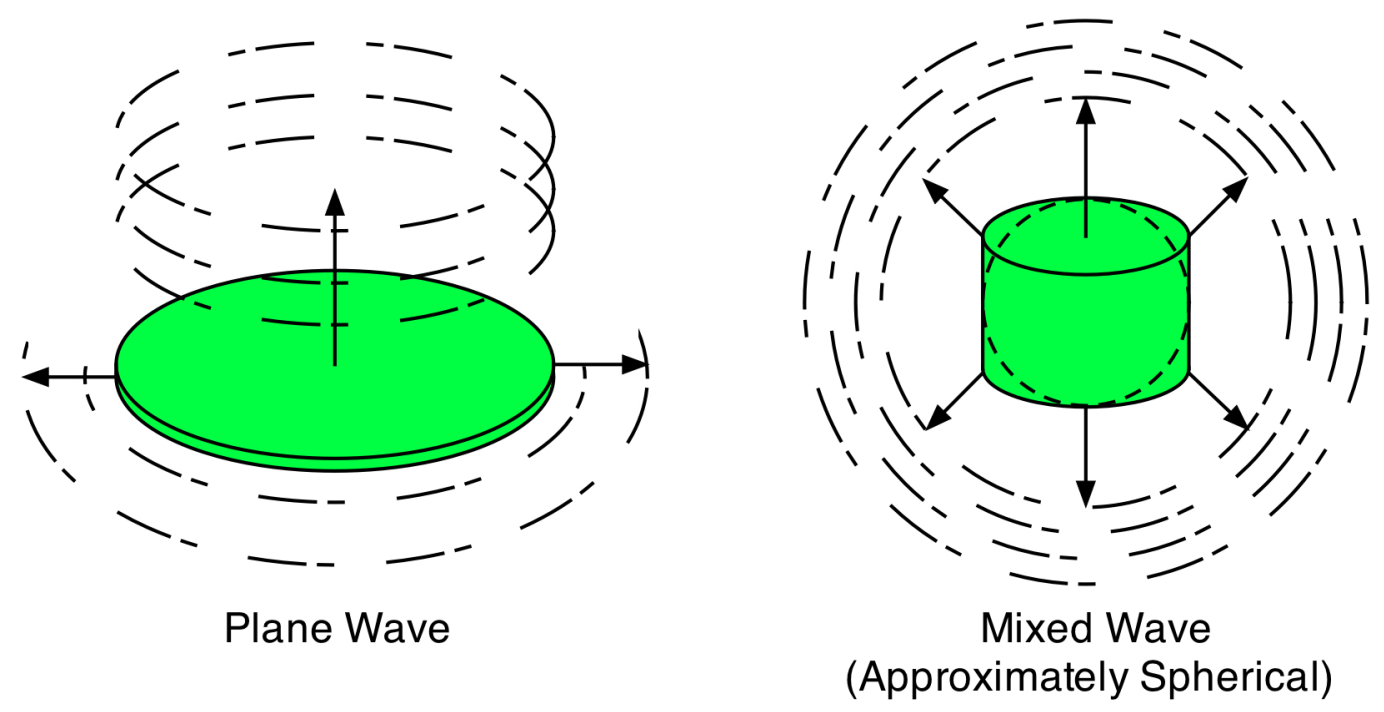

Figure 2.9: This is a diagram of an acoustic plane wave versus a mixed wave (spherical).

Since the energy under TIRPAS excitation is primarily contained in both a forward and backward acoustic wave regardless of the optical absorption of the material being investigated, TIRPAS has a major advantage over traditional photoacoustic spectroscopy in that a larger percentage of the total emitted acoustic energy can be captured for a given signal, since the energy is more directed toward a single source from the excited location. In this way, despite the fact that TIRPAS is inherently less 
sensitive due to the excitation of less material (since the optical penetration depth is less), the technique is more efficient at detecting a material since per unit of material more acoustic waves can be captured for a given absorption.

TIRPAS was originally invented in the early 1980's by Hinoue et al. and others [60-66], in order to evaluate absorption at interfaces for a cyclic voltammetry project. In those days, high powered lasers above $1 \mathrm{~W}$ per pulse were very expensive and difficult to come by, and therefore, the original inventors abandoned increasing the sensitivity of TIRPAS and other uses of the technology in favor of more sensitive technologies that were more appropriate. Since the 1980's, there have been two major sources of improvement in the technology in terms of sensitivity and utility. Notably, one of the improvements in sensitivity combines TIRPAS with a metallic layer to generate surface plasmons generated by the interaction of the light with an appropriately designed layer on a prism of interest. This method is called SPRPAS, or Surface Plasmon Resonance Photoacoustic Spectroscopy.

\subsubsection{Surface Plasmon Resonance Photoacoustic Spectroscopy}

Surface Plasmon Resonance Photoacoustic Spectroscopy was invented by T. Inagaki et al. [67] in the early 1980's alongside the development of TIRPAS by Hinoue et al. In contrast to TIRPAS where the primary mode of excitation is from the absorption of the evanescent field into absorbers close to the interface between a prism and a material of interest, in SPRPAS, the primary mode of excitation is from electron resonance absorption from excitation through a pulsed laser beam into the surface of a metal layer deposited on the prism surface. In this way, SPRPAS, increases the sensitivity of the TIRPAS technique by measuring the non-radiative decay of 
the surface plasmons that form into an acoustic wave for detection. The SPRPAS technique has been used for the fundamental investigation of surface plasmons [67-84], for the measurement of Langmuir-Blodgett films $[85,86]$, and for the detection of nicotine in cigarette smoke [87].

\subsubsection{Modern TIRPAS with Q-switched Lasers}

Goldschmidt et al. [88] renewed interest in the technology by publishing a proceedings paper in a non-refereed journal regarding the use of TIRPAS with modern qswitched ND:YAG based lasers. Specifically, Goldschmidt et al. used nanosecond pulsed ND:YAG lasers with peak powers $\sim 1$ megawatt for the excitation of the chromophores with the evanescent field. This million fold increase in peak power allowed for the TIRPAS technique to become a useful tool for evaluating the absorption of a variety of materials at interfaces. In 2012, Goldschmidt et al. used the technology to show the potential for the early detection of malarial infection [89] by using an optically analogous nanomaterial known as $\beta$-hematin. The main advantage of modern TIRPAS with higher peak power excitation is its inherently high sensitivity for absorbing materials. With the high sensitivity it is possible to use TIRPAS for biosensing small concentrations of important biomarkers in the body for early detection of various disease states and conditions. 


\section{Chapter 3}

\section{Modern TIRPAS and Biosensing}

Evanescent field sensing methods are currently used to detect many different types of disease markers [90] and biologically important chemicals such as the HER2 breast cancer receptor. Hinoue et al. used Total Internal Reflection Photoacoustic Spectroscopy (TIRPAS) as a method of using the evanescent field to detect an optically opaque dye at a sample interface. Although their methods were successful at detecting dyes, the results at that time did not show a very practical spectroscopic technique, which was due to the less than typical sensitivity of TIRPAS as a spectroscopy modality given the low power ( $\sim 0.5$ milliwatt) lasers being used. Contrarily, we have used an ND:YAG laser with a five nanosecond pulse that gives peak power of 1 megawatt coupled with the TIRPAS system to increase the sensitivity of this technique for biological material sensing. All efforts were focused on the eventual detection of the optically absorbing material, hemozoin, that is created as a byproduct of a malarial infection in blood. We used an optically analogous material, $\beta$-hematin, to determine the potential for detection in the TIRPAS system. In addition, four properties that 
control the sensitivity were investigated to increase understanding about the sensor's function as a biosensing method.

\subsection{Evanescent Sensing}

Techniques using the evanescent field have become extremely useful in the past few years. Sensors have been created to detect a wide variety of materials such as to detect 2,4-dinitrotoluene [91], pesticides [92], pathogens [93], nucleic acids [94], gases [95], and disease markers such as the HER2 breast cancer marker [96]. Even lab on a chip microring lasers $[97,98]$ have been created using evanescent field techniques. These sensors function by measuring the interaction of the evanescent field, a nonpropagating near field optical wave, with the target.

In the early 1980's, Hinoue et al. [60-64,99, 100] developed a total internal reflection photoacoustic spectroscopy (TIRPAS) technique to detect dyes in a sample that used the evanescent field to create acoustic waves. Unfortunately, the sensitivity of the device was limited to the detection of highly absorbing dyes that could easily be seen with the eye and was unable to provide a meaningful detection of biological analytes due to the lack of high peak-power lasers. This technique was modified and expanded in order to excite the samples under 1 megawatt peak-power conditions as opposed to the high speed beam chopper with a 0.5 milliwatt continuous wave laser used previously. This giga fold increase in peak-power allows for the detection of microgram quantities of $\beta$-hematin that brings the TIRPAS technology into the useful realm of biological analyte sensing.

Current commercial absorption-based evanescent field biosensors, such as internal 
reflection spectroscopy elements pioneered by Harrick [101], are problematic with low absorbance materials. In internal reflection spectroscopy the measured absorption is determined from the difference between a reference reflection with no sample and the sample's reflected light. This difference is often very small for nearly optically transparent materials in the visible wavelengths. Consequently, complicated and expensive multiple internal reflection systems must typically be created to overcome this limitation. Fortunately, however, since TIRPAS generates an active source of ultrasound with the interaction between the analyte and the evanescent field and there is no inherent ultrasonic background during sampling. Any absorption that can be distinguished by an acoustic transducer will result in detection of a low absorption analyte without the need for a reference reflection. It is important to mention that this technology of generating acoustic waves from total internal reflection is completely distinct from Paltauf et al. [102], who used a glass/sample interface to detect acoustic waves by the local disturbance in the index of refraction from the acoustic waves. Conversely, in TIRPAS, the acoustic wave occurs from the absorption of the evanescent field at the glass/sample interface by the sample.

\subsubsection{Malaria Analytes}

Hemozoin is a chemical byproduct found in human blood that has been infected with malaria. The formation of hemozoin by the malaria parasite Plasmodium falciparum begins once the parasite has infected an erythrocyte of the host. This stage of disease development is characterized by a period of growth for the parasite that is fueled by amino acids of the host cell's cytoplasmic hemoglobin broken down inside a digestive vacuole [103]. The byproduct of hemoglobin digestion is molecular heme, a com- 
pound that harms the parasite by inhibiting enzymes, peroxidizing membranes, and producing oxidative free radicals. Plasmodium falciparum is incapable of digesting heme, and must resort to polymerizing heme into hemozoin, an inert molecule made of heme monomers joined to one another by iron-carboxylate bonds. Infection causes the erythrocyte to eventually rupture and hemozoin is released into the blood stream, where some of it is engulfed by leukocytes via phagocytosis while some of it remains in the bulk blood.

Due to the difficulty in obtaining samples of hemazoin testing with an optically analogous $[104,105]$ material was preferred for these experiments. $\beta$-hematin is a viable analogue for hemozoin in detection systems based on spectroscopic methods, such as TIRPAS, because $\beta$-hematin has the same optical and structural properties as hemozoin [106]. $\beta$-hematin was used for testing the TIRPAS system due to the availability and cost compared to actual hemozoin crystals. As a synthetically derived material, $\beta$-hematin is created by the precipitation of haemin in an acetate buffer [106].

The reasonably high optical absorption of $\beta$-hematin in the visible spectrum allows for the use of a standard laser harmonic wavelength, $532 \mathrm{~nm}$, for photoacoustic excitation. In addition, the $100 \mathrm{~nm} \times 300 \mathrm{~nm}$ average size of $\beta$-hematin is on the order of the evanescent field penetration depth in the TIRPAS experiments. This should give rise to little background signal since the evanescent field cannot penetrate into larger absorbing structures such as red blood cells that are at least an order of magnitude larger than the crystals. $\beta$-hematin and chlorazol black dye, two optically similar materials, are used to show the potential of TIRPAS as a biosensor for the detection of malaria, while advantages and disadvantages of the system with regards to its detec- 
tion capability are also discussed. Additionally, through the angle experiments and previous theory, the shape and size of the photoacoustic effect is shown to be related to absorption, index of refraction, and optical penetration depth. These variables affect any type of photoacoustic spectroscopy by changing the pressure maxima and distribution, however the exact understanding of how the excitation under TIRPAS conditions functions was more completely understood with the following experiments.

\subsection{Materials and Methods}

A schematic of the TIRPAS device is visualized by Figure 3.1.

\subsection{Experimental Setup}

All testing was performed on the same hybrid TIRPAS/oblique photoacoustic excitation setup. Both angle experiments were accomplished by selectively blocking the beam with a spatial filter as explained in Section 3.3.4. The dilution experiment was performed by spatial filtering the beam to leave a laser beam filtered below the critical angle. This left an excitation beam that was a semi-circular spot undergoing TIRPAS excitation at the highest radiant exposure and penetration depth available for TIRPAS in this setup.

\subsubsection{Laser and Optical Fiber}

A Q-switched ND:YAG laser (Surelite I-20 doubled to $532 \mathrm{~nm}$, Continuum, Santa Clara, California) was coupled through $1000 \mu m$ 0.37 NA optical fiber (BFH37-1000, 
Thorlabs, Newton, New Jersey). The laser was pulsed at $20 \mathrm{~Hz}$ at a duration of $5 \mathrm{~ns}$ with energies ranging from 5-6 mJ. The light from the optical fiber was collimated for TIRPAS excitation. We used $100 \mathrm{~mm}$ (LA1509, Thorlabs, Newton, New Jersey), $50 \mathrm{~mm}$ (LA1131, Thorlabs, Newton, New Jersey), and -30 mm (LC2679, Thorlabs, Newton, New Jersey) lenses to collimate the laser light from the optical fiber.

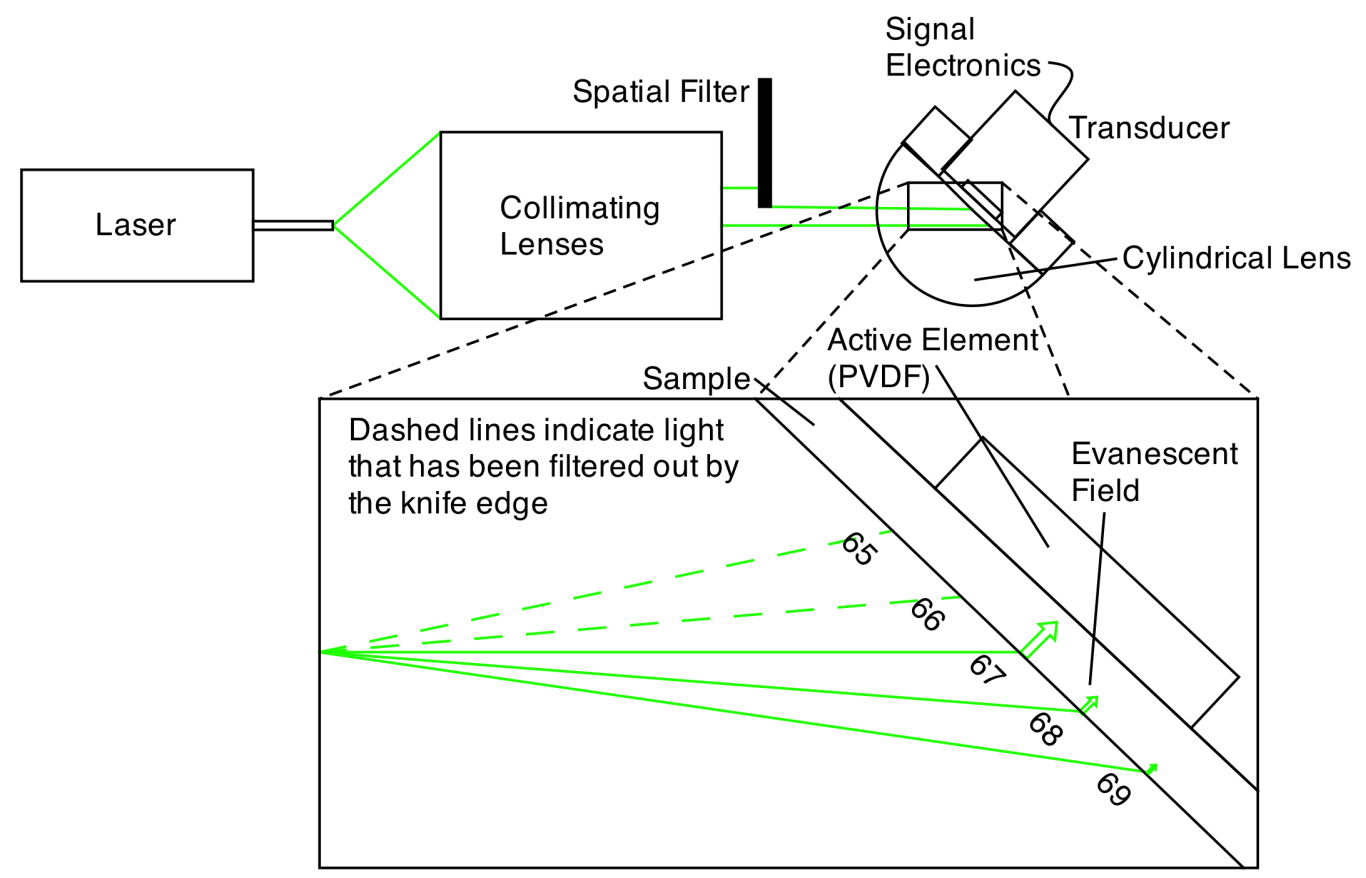

Figure 3.1: This is a schematic of the setup for TIRPAS and how the oblique photoacoustic excitation and TIRPAS excitation differ with angle.

\subsubsection{Sample Holder and Transducer Electronics}

The hemicylindrical lens was mounted to a rotational stage and to an XYZ stage (481-A, Newport, Irvine, California and LT3, Thorlabs, Newton, New Jersey) that allowed the hemicylindrical lens to be adjusted to position the total internal reflected 
spot as well as to change the angle of incidence of the incoming laser light. The voltage response of a polyvinyldiene fluoride (PVDF) transducer was amplified by a $350 \mathrm{MHz}$ preamplifier (SR445A, Stanford Research Systems, Sunnyvale, California). The amplifier was then attached to a $200 \mathrm{MHz}$ four-channel digital storage oscilloscope (TDS 2024B, Tektronix, Beaverton, Oregon) through $50 \Omega$ coaxial cable (2249-C-24, Pomona, Everett, Washington). A silicon photodiode (DET10A, Thorlabs, Newton, New Jersey) was used to trigger the oscilloscope.

\subsubsection{Ultrasonic Detector}

A gold coated PVDF transducer was used as the acoustic sensor to detect ultrasonic waves. This sensor was similar to the designs used in Viator [107] except that the element was not etched and a layer of silicone was added to protect the sensing material and to prevent the sample from staining the transducer. This coating did not introduce significant reflections due to its near acoustic impedance match with the sample that was mostly water. The acoustic impedance of the silicone was 1.1 MRayls as compared to water's 1.5 MRayls. At normal incidence this boundary gives a reflection coefficient of $\sim 2 \%$ and is explained in more detail in A.1. In addition, we used a brass mask to create an approximately $0.5 \mathrm{~cm} \times 0.5 \mathrm{~cm}$ square active area in the center of the transducer to be used as the detection area.

\subsubsection{Spatial Filter}

Harrick [101] suggests a method of collimating the output to the interface by equation

$d=\frac{r}{n_{1}-1}$. where $d$ is the distance of the light source to the prism, $r$ is the radius of 
the lens, and $n_{1}$ is the index of refraction of the hemicylindrical lens. Unfortunately, due to the large radius of the lens and the large numerical aperture of the optical fiber, 0.37 , less than $1 \mathrm{~mJ}$ of energy was possible for TIRPAS excitation under this

configuration. Therefore, $d=\frac{r}{n_{1}-1}$ was not used and a spatial filter was used to block selected angles of excitation for the TIRPAS and photoacoustic spectroscopy experiments to compensate for the lack of collimation at the interface. An XYZ stage (PT3, Thorlabs, Newton, New Jersey) holding a spatial filter was used between light undergoing TIRPAS and oblique incidence photoacoustic excitation respectively as is seen in Figure 3.1. Since the optical setup caused a significant divergence angle across the beam, angles of light were blocked under photoacoustic excitation when TIRPAS was being studied and were blocked under TIRPAS excitation when oblique photoacoustic excitation was being studied. This setup introduced a continuum of angles that collectively gave rise to the overall photoacoustic signal in each experimental case.

\subsubsection{TIRPAS and $\beta$-Hematin}

The microstructure of $\beta$-hematin is an approximately $100 \mathrm{~nm} \times 300 \mathrm{~nm}$ crystalline rod. The crystals have the ability to create a hydrogen bond to neighboring $\beta$-hematin crystals that allows for the crystals to aggregate into larger macrocrystals that may exceed several micrometers in size. The size of a typical red blood cell is several micrometers, at least an order of magnitude larger than the dispersed $\beta$-hematin crystals. The size of $\beta$-hematin is ideal for photoacoustic detection through TIRPAS because the crystals are approximately the same size as the optical penetration depth of the evanescent field. Therefore, absorption from hemoglobin inside red blood cells 
will be reduced by appropriately choosing the optical penetration depth in TIRPAS by adjusting the angle of excitation.

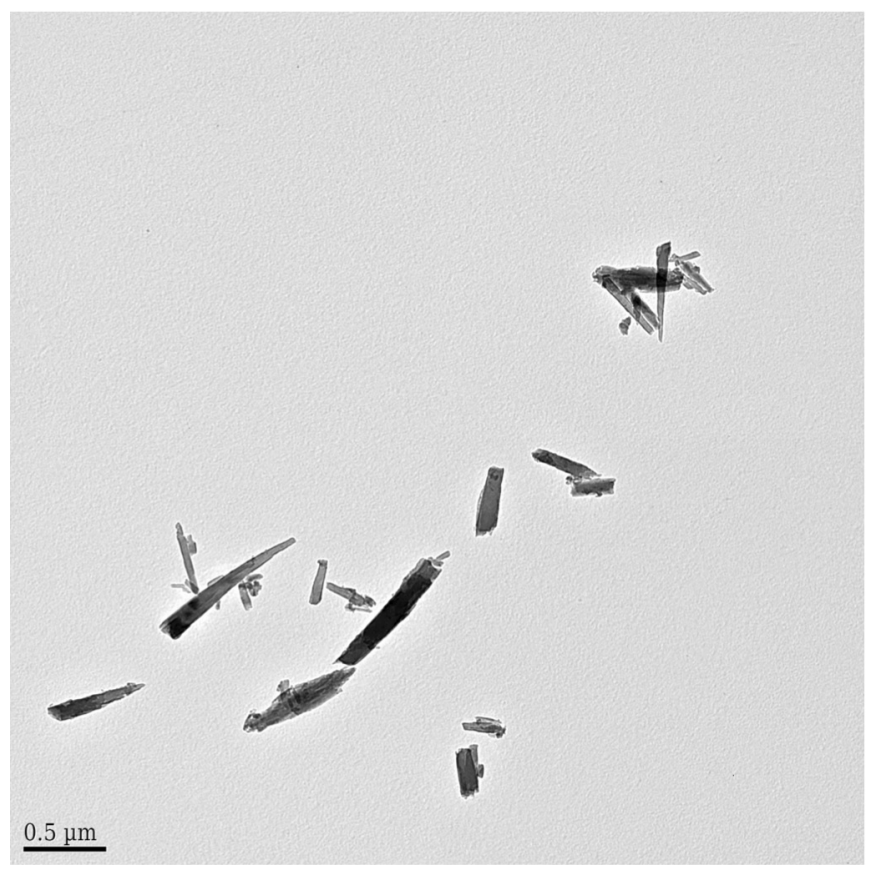

Figure 3.2: Transmission electron microscopy of $\beta$-Hemtain reveals the crystal size and shape.

\subsection{6 $\beta$-Hematin Synthesis}

$40 \mathrm{~g}$ of $\mathrm{NaOH}$ pellets were dissolved into $60 \mathrm{ml}$ of stirred distilled water to create a $12.9 \mathrm{M} \mathrm{NaOH}$ solution while covering the beaker to prevent evaporation. While rapidly stirring $75 \mathrm{ml}$ of $17.1 \mathrm{M}$ glacial acetic acid $12.9 \mathrm{M} \mathrm{NaOH}$ was added drop-wise until the $\mathrm{pH}$ of the titrated buffer solution was 4.5. Distilled water was added until the total solution volume became $100 \mathrm{ml} .34 \mathrm{ml}$ of $0.1 \mathrm{M} \mathrm{NaOH}$ solution was heated to $60^{\circ}$ C. $175.5 \mathrm{mg}$ of haemin (Sigma-Aldrich, St. Louis, MO) was dissolved in the 
$\mathrm{NaOH}$ solution while stirring the covered beaker. $20.7 \mathrm{ml}$ of the acetic acid buffer was heated to $60^{\circ} \mathrm{C}$. The haemin solution was neutralized with $3.4 \mathrm{ml}$ of $1 \mathrm{M} \mathrm{HCl}$ solution. After stirring for 5 minutes the $20.7 \mathrm{ml}$ of acetic acid buffer was added to the neutralized haemin solution. The resulting solution was covered and stirred at $60^{\circ} \mathrm{C}$ for 2 hours. The solution was then put in an ice bath for 10 minutes. The cooled solution was filtered over $8.0 \mu \mathrm{m}$ nitrocellulose filter paper (Millipore, Billerica, MA) and the filtered precipitate was washed with distilled water and dried for 5 minutes. The solid $\beta$-hematin was desiccated for 48 hours. Finally, $\beta$-hematin was stored in PBS suspension away from light exposure at $4^{\circ} \mathrm{C}$ until use.

\subsubsection{Critical Angle Analysis}

The divergent beam allowed for a simple visual determination of the critical angle as seen in Figure 3.1. Using 1.5+ OD safety goggles the reflected spot was observed undergoing TIR from a scattering surface irradiated by the light that had exited the hemicylindrical lens. The scattered laser spot had two visibly important components. One side of the spot is visibly darker due to the increased loss from the reflected light transmitting through the prism/sample interface due to the continuum of divergence angles. The other side is visibly brighter and is indicative of the reflected light that is undergoing evanescent interaction at the prism/sample interface. The interface between the brighter and darker sections of the spot indicates the reflected section of light where the critical angle occurs. Changing the angle of the hemicylindrical lens relative to the incident laser beam shifts the position of this line and thus the physical proportion of the reflected light that is undergoing TIRPAS and oblique

photoacoustic excitation respectively. The resulting incident light is spatially filtered 
with the aid of the reflected light to make the measurements of TIRPAS and oblique photoacoustic excitation independent of each other as shown in Figure 3.3.
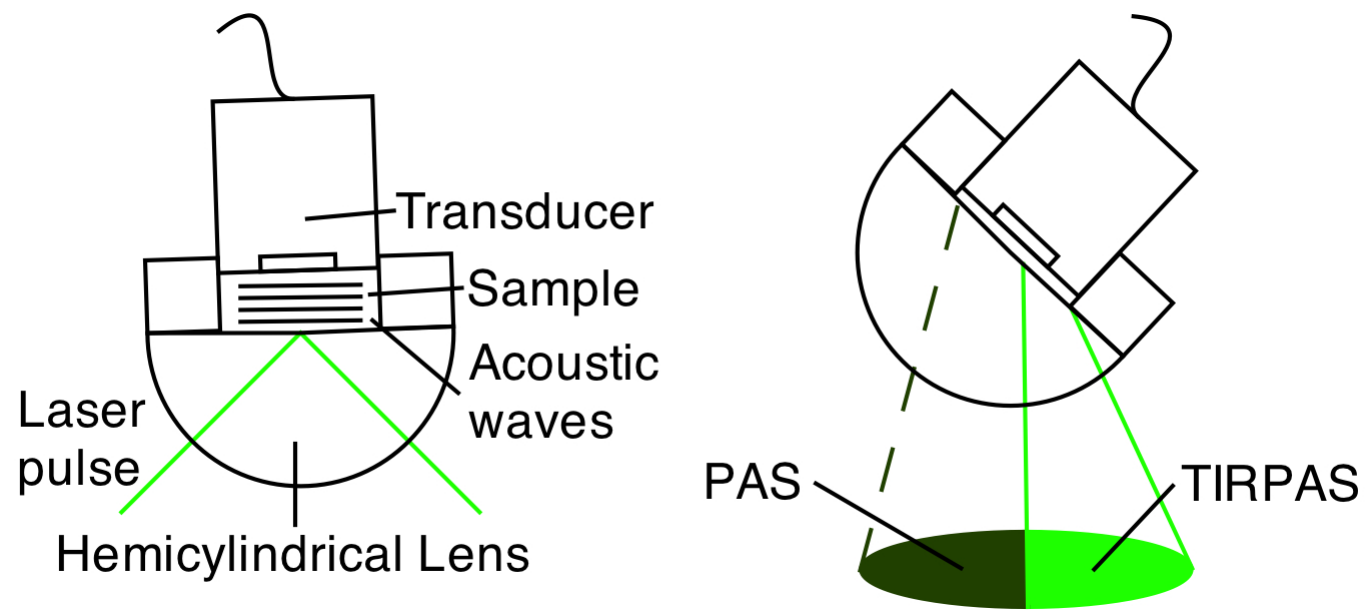

Figure 3.3: Left: This figure shows the experimental setup for TIRPAS. Right: This figure shows reflected light after having interacted with the prism/sample interface. The critical angle is represented by the transition between the brighter and darker reflected portions of light. The darker shade represents the part of the beam under oblique photoacoustic excitation and the brighter shade represents the part of the beam under TIRPAS excitation

\subsubsection{Angular Spectrum: Chlorazol Black}

Chlorazol Black with an absorption coefficient of $55 \mathrm{~cm}^{-1}$ at $532 \mathrm{~nm}$ was used in order to assume a low absorption condition, less than $10 \%$ absorption per interaction, to simplify the equations as stated in Harrick [101]. The condition of less than $10 \%$ absorption per interaction is important because greater absorption may lead to nonlinear optical effects that may lead to deviation from standard Beer's law and thus deviation from expected light interaction. The angle of the hemicylindrical lens was changed relative to the incident laser light by a rotational stage (481-A, Newport, 
Irvine, California). The experiment was performed five times and the values were averaged to obtain a representative angular spectrum as seen in Figure 3.5.

\subsubsection{Detection Limit of Chlorazol Black}

Serial dilutions of an initial $\mu_{a}=52.5 \mathrm{~cm}^{-1}$ Chlorazol Black solutions were excited at $532 \mathrm{~nm}$. The detection limit was defined by a signal to noise ratio of $\sim 3: 1$. The incident light was spatially filtered to produce the largest excitation area of the laser beam at the critical angle for the samples so that the optical penetration depth of the evanescent field (and thus the PA signal) is the largest under this condition.

\subsubsection{Data Collection}

In order to rapidly analyze the photoacoustic signals recorded on the oscilloscope, a graphical user interface (GUI) was developed in MATLAB to import the data and determine the acoustic penetration depth of a given signal. The program determines the penetration depth as a user-defined percentage of the maximum value of the desired photoacoustic waveform. Using cursor placement, the user is able to define the time domain of the photoacoustic signal prior to the percent-maximum calculation in order to avoid bias due to noise and extraneous acoustic effects that may have been recorded in the data file. The program displays the location of the percentmaximum on the plot of the original waveform in addition to displaying its value for user verification of correct penetration depth. 


\subsection{Evaluation}
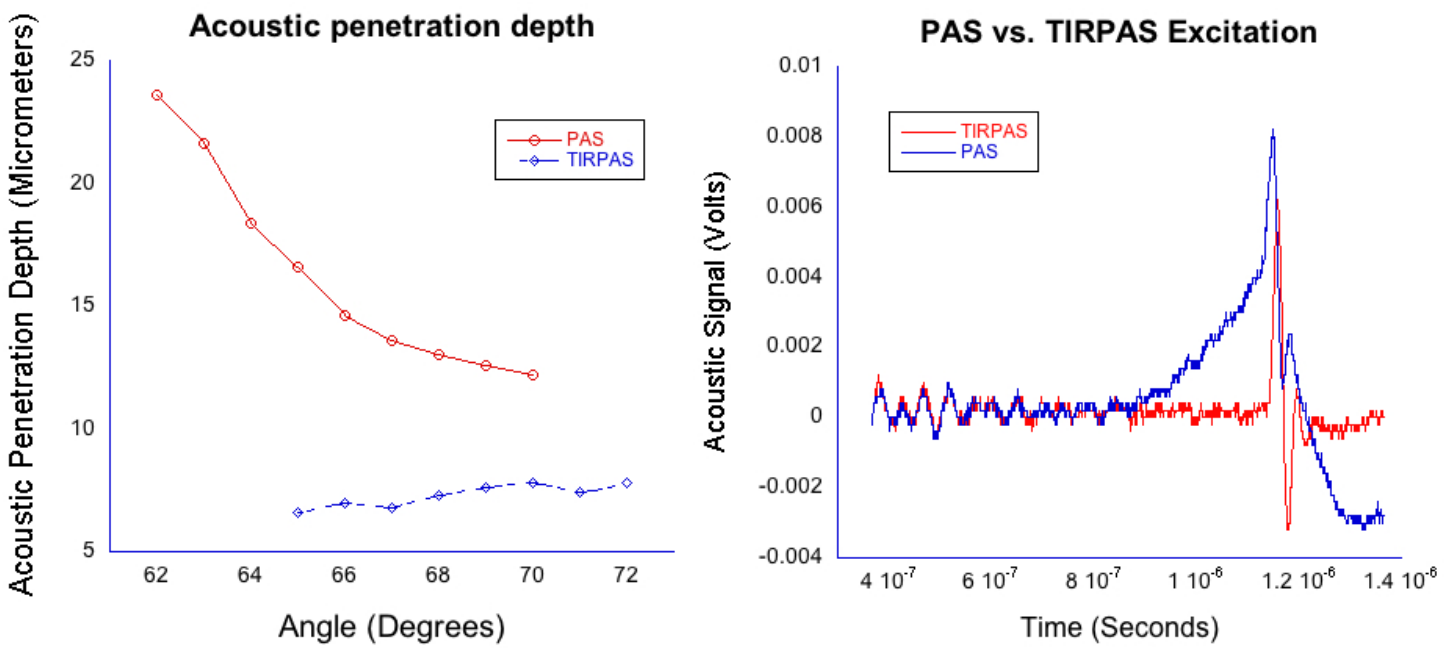

Figure 3.4: Left: Acoustic penetration depth shows that there is an acoustic penetration depth shift versus angle. Right: Signal width differences can be clearly seen between TIRPAS and oblique photoacoustic excitation at the critical angle. Overall, acoustic waves qualitatively seem different between TIRPAS and oblique photoacoustic excitation.

On the right side of Figure 3.4 the comparison between the two types of excitation, TIRPAS and oblique photoacoustic excitation, can be seen. The angular spectrum on the left of Figure 3.4 illustrates how the acoustic penetration depth reduces in magnitude versus an increasing excitation angle. The measured acoustic penetration depth values under TIRPAS excitation and the measured acoustic penetration depth values under oblique photoacoustic excitation never meet in value for this representative sample. The measurements, despite using some of the same angles and both being excited using some form of the photoacoustic effect, show different acoustic penetration depth values where oblique photoacoustic excitation and TIRPAS excitation are contrasted. Of course, since this is a single example there is no measurement of the 
error and therefore it cannot be stated absolutely that this will always be the case, so further testing is needed to confirm any hypothesis regarding differences in the acoustic penetration depth. The graph on the right shows the physical effect of the sample's difference in acoustic penetration depth on single waveforms between oblique photoacoustic excitation and TIRPAS excitation. The TIRPAS waveform rises much faster than its corresponding oblique photoacoustic excitation counterpart.
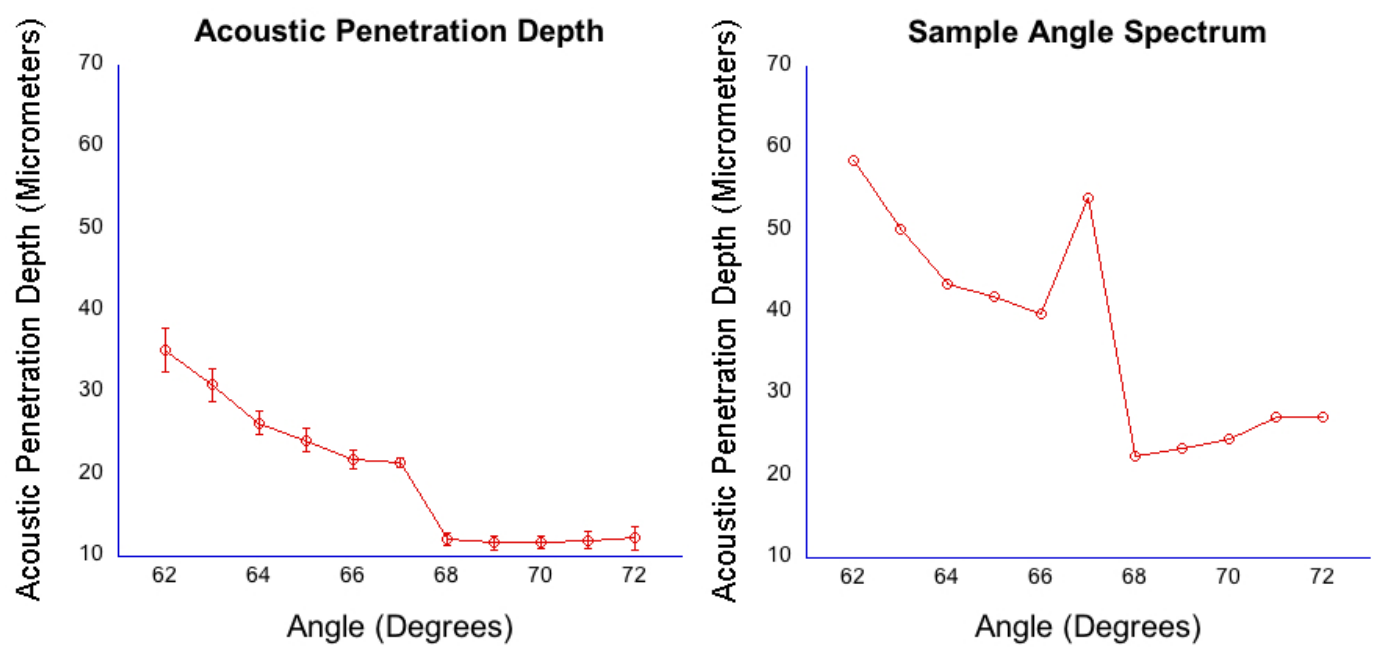

Figure 3.5: Left: Four angular spectra as seen in Figure 3.4 were averaged to create a point wise averaged spectra graph. Right: An outlier in the angle measurement is displayed for completeness. As can be seen in both the outlier and the averaged graph there is a visible transition in acoustic penetration depth at $\sim 67$ degrees. The reduction corresponds to the theoretical optical penetration depth reduction expected from oblique photoacoustic excitation and TIRPAS excitation.

On the left side of Figure 3.5 the point wise average of penetration depths at various angles are used to illustrate a comparison between the acoustic penetration depth in both oblique photoacoustic excitation and TIRPAS excitation as shown. There is a substantial reduction in measured acoustic penetration depth at the critical angle between oblique photoacoustic excitation and TIRPAS excitation. The right side 
of Figure 3.5 shows an anomalous reading that the author believes may have been caused by setup movement during the experiment, but is displayed for completeness. A detection limit experiment was performed with a solution of Chlorazol black dye to estimate the approximate detection limit of the system. The approximate detection limit was $\sim 1.1 \mathrm{~cm}^{-1}$. Such a low absorption would be difficult to detect using oblique photoacoustic excitation due to the very large optical path length needed for excitation.
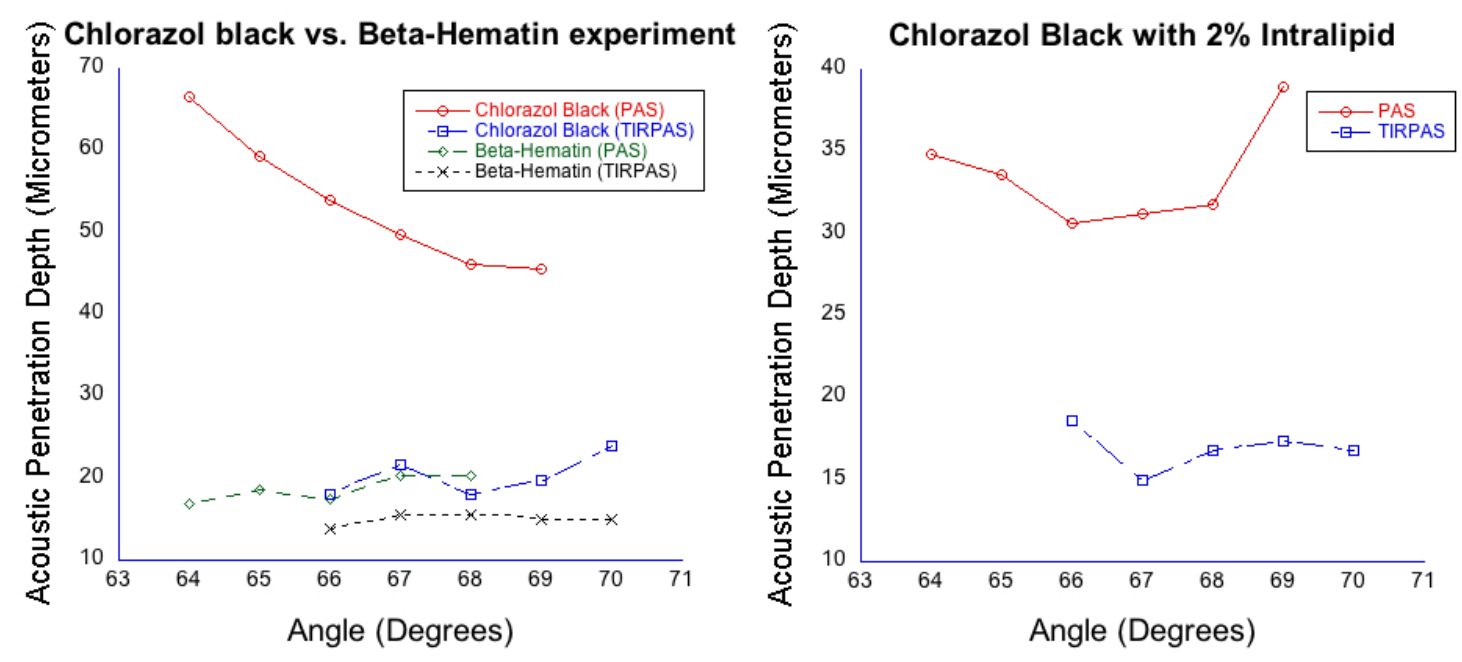

Figure 3.6: Left: This figure shows an angular spectrum with $\beta$-hematin and Chlorazol black under oblique photoacoustic excitation and TIRPAS excitation. Right: This figure shows an angular spectrum of the $2 \%$ Intralipid/Chlorazol black solution that was made to approximate the oblique photoacoustic excitation optical penetration depth obtained by $\beta$-hematin.

Figure 3.6 shows the results of an experiment to determine the penetration depth of an optically absorbing and optically scattering material. The angular spectrum, without point wise averaging, on the left shows the acoustic penetration depth of standard chlorazol black dye and $\beta$-hematin for both oblique photoacoustic excitation and TIRPAS excitation. The $\beta$-hematin acoustic penetration depth values are much closer 
in value for both oblique photoacoustic excitation and TIRPAS excitation. The figure on the right shows Chlorazol Black with a $2 \%$ intralipid optically scattering solution. Both TIRPAS and oblique photoacoustic excitation measured acoustic penetration depths are much closer to each other, similar to the figure on the left for $\beta$-hematin despite the only difference being the addition of optical scattering.

\subsection{Discussion}

\subsubsection{How to Relate Optical Absorption to Refractive Index}

It is well known for both evanescent field sensing [108-111] and for TIRPAS based sensing $[60-66,88,89,99,100]$ that the electric field decays with an exponential according to

$$
E_{z}=E_{0} e^{-z / \delta_{p}},
$$

where $E_{z}$ and $E_{0}$ refer to the electric field intensity at a sample depth, z, and initial intensity at the interface $(z=0)$, respectively, with $\delta_{p}$ as the optical penetration depth. The ability for the light to be absorbed into an analyte very close to the interface is dependent upon four fundamental properties: penetration depth, electric field strength, sampling area, and how well the sample and prism indicies are matched [101]. Since the photoacoustic effect is caused by absorption of photons into materials that causes localized thermoelastic expansion to induce an acoustic wave, the penetration depth is one of the factors that controls the sensitivity of TIRPAS since this determines photonic absorption into a material and thus the intensity of the resulting acoustic 
wave. The penetration depth is controlled by the following factors given by $[112,113]$

$$
\delta_{p}=\frac{\lambda_{1}}{2 \pi\left(\sin ^{2} \theta-n_{21}^{2}\right)^{1 / 2}},
$$

where $n_{21}=\frac{n_{2}}{n_{1}}, n_{2}$ and $n_{1}$ are the indices of refraction of the hemicylindrical lens and the sample, where $\theta=$ angle of excitation, and $\lambda_{1}=\frac{\lambda}{n_{1}}$ according to Muessig et al. [66] The laser used did not have a short enough pulse duration to fall within a stress confined condition, however, a reasonable proportion to explain the amount of power absorbed can still be obtained when the underlying causes of the absorption and the relationship of the absorption to the index of refraction, are considered.

\subsubsection{Optical Absorption Under TIRPAS Excitation}

In Muessig et al. [66] the authors describe an equation to relate the absorbed power to both the penetration depth and the intensity of the electric field. A similar expression can be obtained by substituting the electric field with an average of the parallel and perpendicular polarizations of the electric field. This does not completely explain our results due to the assumption that polarization interaction does not change with the angle of incidence. However, it does give a general overview to the interaction if each polarization is assumed to contribute equally to the absorbed power. If the terms $E_{\|}^{\prime}$ and $E_{\perp}^{\prime}$ are known in the experiment being conducted their proportional contribution can be calculated by the amount each contributed to the acoustic signal. The variables relating to photoacoustic pressure can be explained by

$$
P \propto \delta_{p}\left[\frac{\left[E_{\|}^{\prime}\right]^{2}}{2}+\frac{\left[E_{\perp}^{\prime}\right]^{2}}{2}\right]
$$


where $E_{\|}^{\prime}$ is the electric field intensity of the parallel polarization and $E_{\perp}^{\prime}$ is the electric field intensity of the perpendicular polarization and $P$ is the acoustic pressure. Absorption is directly related to the amount of acoustic pressure generated in a sample under photoacoustic excitation. From Muessig et al. [66], it has been shown that the electric field parallel to the interface is

$$
E_{\|}^{\prime}=\left(E_{x}^{\prime 2}+E_{z}^{\prime 2}\right)^{\frac{1}{2}}
$$

where the individual components $E_{x}^{\prime}$ and $E_{z}^{\prime}$ are

$$
E_{x}^{\prime}=\frac{2 E_{\|}\left(\sin ^{2} \theta-n_{21}^{2}\right)^{\frac{1}{2}} \cos \theta}{\left(1-n_{21}^{2}\right)^{\frac{1}{2}}\left[\left(1+n_{21}^{2}\right) \sin ^{2} \theta-n_{21}^{2}\right]^{\frac{1}{2}}}
$$

and

$$
E_{z}^{\prime}=\frac{2 E_{\|} \sin \theta \cos \theta}{\left(1-n_{21}^{2}\right)^{\frac{1}{2}}\left[\left(1+n_{21}^{2}\right) \sin ^{2} \theta-n_{21}^{2}\right]^{\frac{1}{2}}}
$$

respectively. In addition, the electric field perpendicular to the surface is

$$
E_{\perp}^{\prime}=\frac{2 E_{\perp} \cos \theta}{\left(1-n_{21}^{2}\right)^{2}} .
$$

By using (3.3)-(3.4) and substituting them into (3.2) we get 


$$
\begin{aligned}
& P \propto \frac{\lambda_{1}}{2 \pi\left(\sin ^{2} \theta-n_{21}^{2}\right)^{\frac{1}{2}}} \times \\
& {\left[\frac{\left\{\left[\frac{2 E_{\|}\left(\sin ^{2} \theta-n_{21}^{2}\right)^{\frac{1}{2}} \cos \theta}{\left(1-n_{21}^{2}\right)^{\frac{1}{2}}\left[\left(1+n_{21}^{2}\right) \sin ^{2} \theta-n_{21}^{2}\right]^{\frac{1}{2}}}\right]^{2}+\left[\frac{2 E_{\|} \sin \theta \cos \theta}{\left(1-n_{21}^{2}\right)^{\frac{1}{2}}\left[\left(1+n_{21}^{2}\right) \sin ^{2} \theta-n_{21}^{2}\right]^{\frac{1}{2}}}\right]^{2}\right\}}{K_{1}}+\frac{\left[\frac{2 E_{\perp} \cos \theta}{\left(1-n_{21}^{2}\right)^{2}}\right]^{2}}{K_{2}}\right],}
\end{aligned}
$$

where $\theta$ is the angle of incidence, $n_{21}=\frac{n_{2}}{n_{1}}, \lambda_{1}=\frac{\lambda_{0}}{n_{1}}, K_{1}$ and $K_{2}$ are the constants to indicate the proportion of each polarization used in excitation; in this case $K_{1}=K_{2}=2$ since each polarization is assumed to contribute half the photoacoustic response to the system, and $E_{\|}$and $E_{\perp}$ are the electric field intensities for parallel and perpendicular polarization relative to the prism. Beyond pressure, the effective thickness of the evanescent field is controlled by four factors, electric field intensity, penetration depth, index matching of the prism/sample interface, and the sampling area. The equation above shows variables for three of the four factors, penetration depth, electric field, and index matching that agrees well with our experiments. The sampling area should be included as a multiplication constant for the above equation since the acoustic pressure should be proportional to the area of excitation.

\subsubsection{Acoustic Plane Waves in TIRPAS}

In contrast to normal incidence photoacoustic excitation, our observation was that every waveform regardless of the optical absorption seemed to be a bipolar plane wave. This observation can be understood with a more thorough analysis of the in- 
teraction between the evanescent field and the target absorber. Plane waves can be formed in the acoustic under normal incidence photoacoustic excitation. The plane wave assumption is appropriate to apply when the transducer is closer to the incident

absorber than the boundary where $w=\frac{d^{2} \mu_{a}}{8}$, or in the acoustic near field, as found in Viator et al. [59] where $w$ is the distance from the transducer to the near field boundary, $d$ is the beam diameter, and $\mu_{a}$ is the absorption coefficient. Under this condition an acoustic piston is approximated as a plane wave where almost half the acoustic energy generated moves forward toward the transducer. With TIRPAS, a similar effect is observed as seen in Figures 3.4 and 3.5 that can be explained by the fact that the evanescent field only optically penetrates a very small amount, $\sim 500$ $\mathrm{nm}$, into the medium and so an acoustic piston is nearly guaranteed for almost all absorbing materials. The acoustic piston phenomena made detection much less ambiguous than normal incidence photoacoustic excitation where plane waves are only obtained if the sample is significantly absorbing and the spot size is very large according to $w=\frac{d^{2} \mu_{a}}{8}$. In the TIRPAS system an acoustic plane wave will be obtained for all absorbing materials unless the spot size for excitation is as small as the optical penetration depth or thickness of the sample, whichever is smaller, that would necessarily be a diffraction limited beam for excitation in the visible wavelengths.

\subsubsection{Optical Penetration Depth}

The optical penetration depth of the evanescent field exponentially decays away from the interface of total internal reflection. The distance where the evanescent field decays to $37 \%\left(\frac{1}{e}\right)$ of the incident energy is defined as the optical penetration depth and can be used as one factor to predict evanescent field interaction that is described 
by equation $3.1[108,109]$. The penetration depth is important for TIRPAS excitation that uses the interaction of the evanescent field with absorbers to detect the analyte. Physical sizes of analytes can range from an order of magnitude larger to an order of magnitude smaller than the penetration depth of the evanescent field $(\sim 500 \mathrm{~nm})$ in typical biological systems. An example of the large distribution of sizes would be a cell that may be $10 \mu \mathrm{m}$ in size compared to the size of a single hemazoin crystal that is $100 \mathrm{~nm} \times 300 \mathrm{~nm}$. By tuning the penetration depth of the evanescent field by exciting the prism/sample interface at increasing excitation angles, analytes of ever smaller sizes can be detected since the field will interact most strongly with the material based upon its size along with its inherent optical absorption.

\subsubsection{Stress Confinement}

In normal incidence photoacoustic excitation, stress confinement, described in (3.6), is a condition where the pulse duration of incident light is shorter in duration than the time it takes for the induced acoustic wave to propagate away substantially. Under stress confinement the resulting acoustic wave represents how the incident light optically decays through the media. This effect allows for optical properties such as the absorption coefficient to be estimated using an exploratory non-linear least squares curve fitting algorithm as shown in Figure 3.7 from the acoustic wave. This is explained in more detail by Viator et al. [59] and Viator [107].

$$
\tau \leqslant \frac{\delta}{c_{s}}
$$

Equation (3.6) is the stress confinement condition where $\delta=\frac{1}{\mu_{a}}, \mu_{a}$ is the absorption 
coefficient of the sample, and $c_{s}$ is the speed of sound in the sample medium.

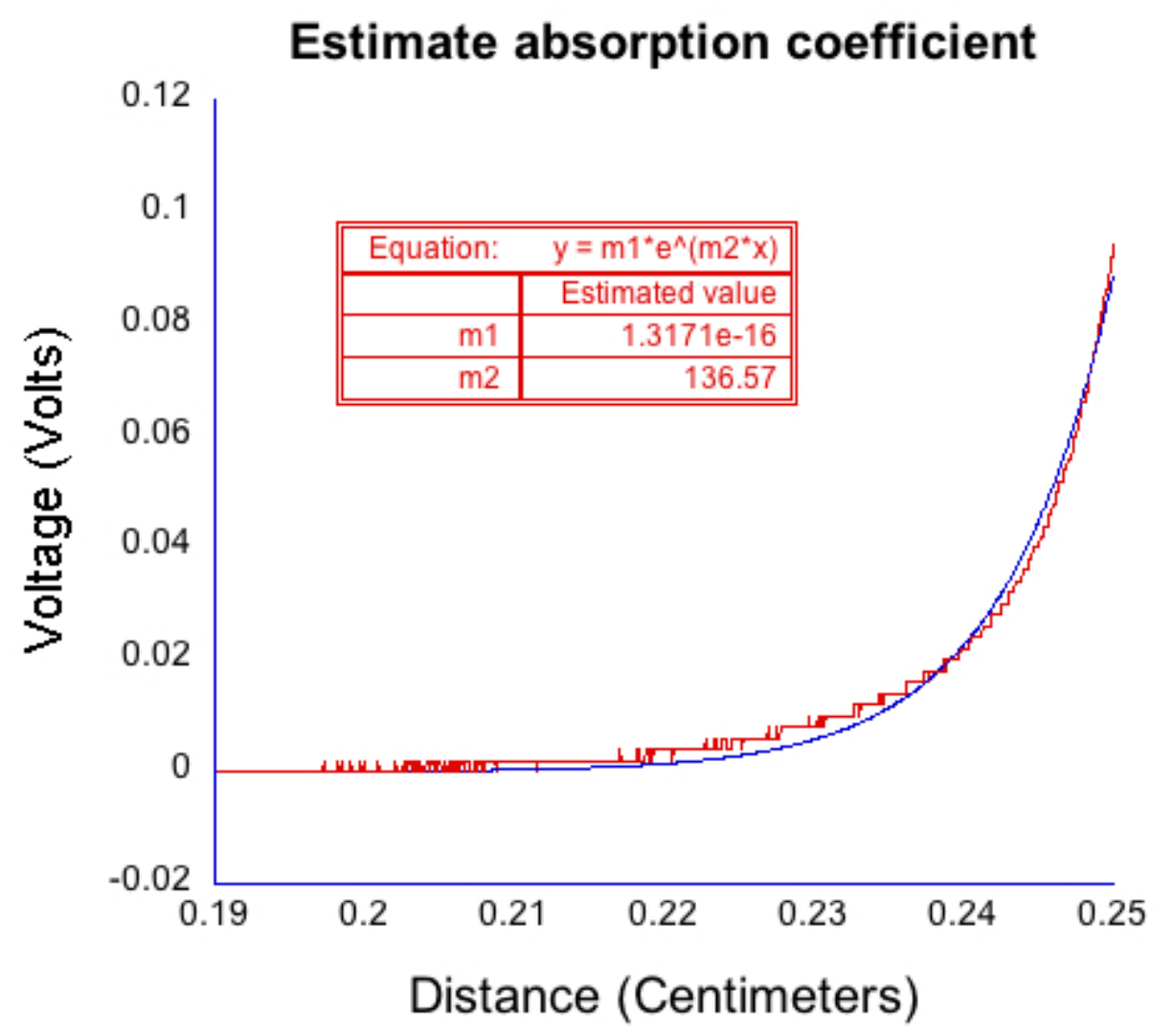

Figure 3.7: A Non-linear exponential least squares curve fit can be used to estimate $\mu_{a}$.

Meeting the stress confinement condition and inputting the acoustic wave in Figure 3.7 and using a non-linear exponential least squares curve it, it is possible to gain some insight into the optical properties such as an estimate for the absorption coefficient of the material of interest. This does not take into account statistical uncertainty, therefore, this is used as more of an exploratory method to get a general idea of the value of the absorption coefficient rather than an exact value for further testing. The author used $\mathrm{m} 1=1$ and $\mathrm{m} 2=150$ for the initial guesses with $0.1 \%$ tolerance and 
more information about this method and experimental setup for collecting this type of acoustic wave can be found in [59]. Assuming that TIRPAS waveforms are stress confined, the experimental evidence should show that the waveforms' penetration depth reduces with a reduction in the evanescent field penetration depth. However, our data shows a reasonably insensitive measurement compared to theoretical values as seen in Figures 3.4, 3.5, and 3.6.

Choi et al. [114], studied how the laser pulse duration can affect the determination of $\mu_{a}$ computed from curve fitting waveforms collected by normal incidence photoacoustic excitation. It has been shown that as the stress confinement condition was no longer satisfied, the $\mu_{a}$ estimation was increasingly underestimated until the limiting value of $\mu_{a}$ is reached. In that case, one would expect the penetration depth, which is the inverse of $\mu_{a}$, to hit a minimum value if the stress confinement condition is not met. Our results seem to agree with non-stress confined photoacoustic excitation, as seen in Figures 3.4 and 3.5. This result suggests that TIRPAS excitation is not in stress confinement and is limited by the duration of the laser pulse. If laser light is delivered to a sample within the stress confinement condition, no substantial acoustic energy has time to propagate away before the pulse duration of the laser ends. Since TIRPAS has a very small penetration depth, $\sim 500 \mathrm{~nm}$, then calculating with $\tau=\frac{\delta}{c_{s}}$ the stress confinement estimate for TIRPAS is at maximum 0.33 nanoseconds around the largest optical penetration depth attainable.

\subsubsection{Signal Acquisition}

Since the penetration depth of the evanescent field, even near the critical angle, is within hundreds of nanometers of the interface, most of the frequency components 
expected under stress confined TIRPAS would be in the low GHz range. Since the duration of the laser pulse was $5 \mathrm{~ns}$, this limited the frequency range to the maximum obtainable via a 5 ns pulse. To approximate this frequency range, we used a simple method [107] and assumed a pulse duration of $5 \mathrm{~ns}$ and used

$$
f=\frac{1}{p_{d}},
$$

where $\mathrm{f}$ is the approximate frequency maximum of the resulting wave and $p_{d}$ is the pulse duration of the laser. The PVDF sensors used were reliable at frequencies up to approximately $100 \mathrm{MHz}$. Far beyond $100 \mathrm{MHz}$, the PVDF does not respond linearly to inputs. The maximum sampling rate of the oscilloscope used was $200 \mathrm{MHz}$ that would not be enough sampling to fully capture the signals appropriately if a picosecond laser were used in order to stay within the regime of stress confinement. However, due to the nanosecond pulse duration mentioned previously, the maximum frequency threshold was far below the maximum frequency of the oscilloscope and therefore, no distortion was expected. In Figure 3.4 the difference in frequency components can be qualitatively inferred when the acoustic penetration depth of oblique photoacoustic excitation and TIRPAS excitation are compared. Of course, a more rigorous statistical evaluation would be needed to show this for certain, however, TIRPAS contains laser pulse limited frequency components due to its optical penetration depth being on the order of a few hundred nanometers. The measured acoustic penetration depth (roughly corresponding to optical penetration depth) of TIRPAS corresponds to a rise time of 5 ns in Figure 3.4. 


\subsubsection{Differences in Penetration Depth}

With the above limitations in mind, it is important to note the differences detected between the TIRPAS acoustic wave and the oblique photoacoustic excitation acoustic wave at compared angles of incidence. Figure 3.5 shows the measured acoustic penetration depth corresponding to the optical penetration depth versus the angle of incidence of the incident light. It should be noted that due to the beam divergence the angles listed are a continuum of angles from the critical angle to the most grazing angle.

The measured acoustic penetration depth under oblique photoacoustic excitation levels off as the incident light is tuned/spatially filtered very close to the critical angle. Incident light exciting any sample will always have a lower measured acoustic penetration depth with larger angles of incidence. At the critical angle the measured acoustic penetration depth reduces to the laser pulse limited acoustic penetration depth for all angles at or beyond the critical angle. This huge reduction in acoustic penetration depth is due to the fact that in oblique photoacoustic excitation the optical penetration depth is solely a function of the absorber's optical properties, but in TIRPAS, the optical penetration depth is a function of the wavelength, index of refraction of the sample and substrate, and the angle of incident light at the prism/sample interface, that gives rise to the evanescent field that substantially limits the optical penetration depth.

\subsubsection{Advantages of TIRPAS}

There are three advantages to TIRPAS compared to conventional photoacoustic spectroscopy and other evanescent field spectroscopies: 
1. Optical size filtering can limit the material being excited.

2. Simple, obvious detection methods are obtained using this method.

3. Taking an optical spectrum is possible with the use of tunable lasers.

With regard to 1 , since the excitation comes from the interaction of the evanescent field with the interface up to only a few hundred nanometers, biological analytes of interest can be size filtered for detection. This type of size filtering utilizing the evanescent field's angular dependent optical penetration depth allows for the analysis of harmful chemicals in biological fluids without the substantial background from cellular components. Relating to 2, TIRPAS produces easily discernible signals since all generated acoustic waves are plane waves as discussed previously. Moreover, the absorbed energy is converted to acoustic energy making the need to change detectors based on wavelength unimportant and therefore, allowing the method to be utilized over the large optical spectrum without expensive or time consuming alterations to the setup $[58,115]$. Finally for 3 , TIRPAS has the potential to determine the absorption spectrum of a material in a similar way to internal reflection spectroscopy as discussed

by Harrick [101]. This type of spectroscopy may be much less expensive due to the lack of need to change detectors as mentioned.

\subsubsection{Optical Scattering of $\beta$-Hematin}

In Figure 3.6, the penetration depth for Chlorazol black is typical for what is expected with oblique photoacoustic excitation and TIRPAS excitation. However, for $\beta$-hematin the same setup parameters provide the same results for TIRPAS but generate very different results for oblique photoacoustic excitation. From transmission 
electron microscopy images of $\beta$-Hematin in Figure 3.2 it is observed that the length of individual crystals is on the order of the wavelength of the incident light used, 532 nm. Due to $\beta$-hematin's size and shape the component for Mie scattering as well as Raleigh scattering should be very strong with the sample's crystal size and incident light wavelength.

Chlorazol black dye was mixed with intralipid at $2 \%(\mathrm{v} / \mathrm{v})$ of the same optical absorption coefficient and was tested as a comparison to $\beta$-Hematin. This mixture increased the optical scattering of the Chlorazol black to visually approximate that of $\beta$-Hematin. In Figure 3.6 the results of this test show that the same trend of the measured acoustic penetration depth under oblique photoacoustic excitation as compared with highly absorbing Chlorazol black solution is no longer observed. Instead a very similar result to the $\beta$-Hematin is observed where the measured acoustic penetration depth changes differently from all absorbing materials along with the overall measured acoustic penetration depths being much closer to those observed in TIRPAS.

This observation can be explained by two factors. First, oblique photoacoustic excitation under stress confinement displays the distribution of photons in a material. Therefore, a scatterer, by changing the distribution of photons, can alter the measured acoustic penetration depth associated with it. Also, the addition of optically scattering material tends to make the optical penetration depth smaller since the distribution of photons is affected by both $\mu_{a}$ and $\mu_{s}$. Specifically, the distribution of photons in Chlorazol black solution can be described by

$$
I=I_{0} e^{-\mu_{a} z} .
$$




$$
I=I_{0} e^{-\left(\mu_{a}+\mu_{s}(1-g)\right) z}
$$

However, if optical scattering is introduced, the effective attenuation of the light is increased due to the addition of the scattering coefficient, $\mu_{s}$, and the anisotropy of the material, $g$ as shown in (3.7).

\subsection{Conclusion}

TIRPAS has the ability to detect Chlorazol black dye at an absorption coefficient of $1.1 \mathrm{~cm}^{-1}$. In addition, the results demonstrate through Figure 3.5 how the acoustic (and by extension optical) penetration depth changes with the angle of incidence. Finally, the angular spectrum of $\beta$-hematin shows the potential for TIRPAS to be used as a biosensor for the early detection of malarial infection. 


\section{Chapter 4}

\section{Photoacoustic Refractometry (PAS/TIRPAS) and Biosensing}

Current methods of determining the refractive index of chemicals and materials, such as ellipsometry and reflectometry, are limited by their inability to analyze highly absorbing or highly transparent materials, as well as the required prior knowledge of the sample thickness and estimated refractive index. Here, we present a method of determining the refractive index of solutions using the photoacoustic effect. We show that a photoacoustic refractometer can analyze highly absorbing dye samples to within 0.006 refractive index units of a handheld optical refractometer. Further, we use myoglobin, an early non-invasive biomarker for malignant hyperthermia, as a proof of concept that this technique is applicable for use as a medical diagnostic. Comparison of the speed, cost, simplicity, and accuracy of the techniques shows that this photoacoustic method is well-suited for optically complex systems. 


\subsection{Introduction}

The refractive index of a material describes the group velocity of light as it propagates through that medium. This property is highly correlated with both the density of the medium, and the absorptive properties of the medium. Using Kramers-Kroenig analysis (mathematical relationships that relate the complex and real parts of a phys-

ical system) it is possible to estimate many material properties, including refractive index. However, in recent years, the legitimacy of this analysis has been questioned for heterogenous biological materials, such as hemoglobin, due to the complex nature of the analysis [116]. Measurement of the refractive index is based upon the fundamental Fresnel equations that describe the refraction, reflection, and transmission of light at an interface between two media with non-matching refractive indices. In recent years, the gold standard of measurement of this property has been either reflectometry or ellipsometry, depending upon various sample properties, such as its transparency, and thickness, as well as overall cost, and the necessary data precision. However, for highly absorbing materials, partially transparent materials, or entirely unknown materials, both techniques demonstrate significant drawbacks that prevent the widespread adoption of refractive index measurements beyond typical materials characterization. Here, we explore a low cost, simple, alternative method based on the photoacoustic effect. We demonstrate that our photoacoustic method, Photoacoustic Spectroscopy/Total Internal Reflection Photoacoustic Spectroscopy (PAS/TIRPAS) refractometry, can be successfully used to determine the refractive index of both dyes, as well as a physiologically relevant concentration of myoglobin $[117,118]$ in phosphate buffered saline. 


\subsubsection{Reflectometry}

Reflectometry is a technique where a polarized beam of light, operating in two linear polarizations, S and P, optically interrogates a sample. The reflected light from the sample is then measured by a photodetector. Both $\mathrm{S}$ and $\mathrm{P}$ polarizations, along with the incident angle, are recorded in order to obtain the real component of the refractive index. Equation (4.1) shows how the refractive index of a material is related to the reflection coefficients and angle of incidence as

$$
n_{R}=\sin \theta_{i}\left[1+\left(\frac{1-\rho}{1+\rho}\right)^{2} \tan ^{2} \theta_{i}\right]^{\frac{1}{2}}
$$

where $\rho=\sqrt{\frac{R_{p}}{R_{s}}}$ and $n_{R}=\frac{n_{2}}{n_{1}}$. Equation 4.1 is used to determine the real part of the refractive index of a material using reflectometry methods [119].

\subsubsection{Critical Angle Analysis}

In a special case, the real component of the refractive index can be determined by measuring the critical angle, or the angle where total internal reflection occurs. This angle can be determined using equation 4.6. This method simplifies the analysis of Fresnel's equations and allows the refractive index of the sample to be found directly, if the refractive index of the substrate, $n_{1}$, and the angle of incidence, $\theta_{1}$, are known.

\subsubsection{Ellipsometry}

Ellipsometry, a closely related technique to reflectometry, can be described with a slight alteration to Equation 4.1. The relative permittivity of a material, $\tilde{\varepsilon}$, can be 
found, and is directly related to the refractive index of a material by (4.2) and (4.3). This is explained in more detail at http://www.rp-photonics.com/refractive_ index.html. The equations are given by

$$
\langle\tilde{\varepsilon}\rangle=\sin ^{2} \phi\left[1+\left(\frac{1-\rho}{1+\rho}\right)^{2} \tan ^{2} \phi\right]
$$

and

$$
\langle\tilde{\varepsilon}\rangle=n^{2}=\kappa
$$

where $\tilde{\varepsilon}$ and $\kappa$ are the relative permittivity of a material, $n$ is the refractive index of the sample material, $\phi$ is the incident angle on the sample, and $\rho=\sqrt{\frac{R_{p}}{R_{s}}}$ is the ratio between the two reflected values for both linear polarizations, $\mathrm{P}$ and $\mathrm{S}$. For simple bulk substrate systems, ellipsometry uses the same equation as reflectometry, albeit formatted differently, to determine the refractive index of a material.

\subsubsection{Limitations}

Each of these methods has limitations. Reflectometry's main limitation lies in the fact that the technique does not take into account surface roughness or optical scattering effects. Therefore, the actual measurement of the refractive index is a combination of the optical contributions from both refractive index and surface or substrate roughness. Additionally, reflectometry typically has less success with materials that have many highly absorbing layers, due to the light being unable to be measured from being completely absorbed. Ellipsometry has similar issues with measuring materials that are highly absorbing for the same reasons. Additionally, ellipsometry has the addi- 
tional limitation of being unable to determine a single optical property independently of the thickness of a material.

This can lead to erroneous results if the operator is not appropriately trained and makes incorrect assumptions or initial guesses for thickness and refractive index in the curve fitting program to determine refractive index and layer thickness. Model fitting in ellipsometry attempts to minimize the mean squared error or (MSE) in order to achieve a global minimum to determine the values of thickness and refractive index at a particular wavelength. More information can be obtained at http://www . jawoollam.com/tutorial_5.html.

Critical angle analysis is highly accurate and inexpensive, however, it requires a prism setup that will be optically complicated and physically large. Additionally, the material of the prism must be of a larger refractive index than the sample in order for the condition for total internal reflection to be fulfilled that poses problems for unknown samples and increases cost.

\subsubsection{The Photoacoustic Effect}

An alternative to all of these methods relies on the photoacoustic effect. The photoacoustic effect is generated by the absorption of light into a sample that induces pressure in the form of an acoustic wave. The simplest equation to describe photoacoustic excitation is equation 4.4 from Viator et al. [59] that describes the pressure distribution of a generated photoacoustic plane wave. The magnitude of the resulting

acoustic wave relies directly upon the optical absorption coefficient of the material which can be explained by 


$$
p_{0}(z)=\mu_{a} \Gamma H_{0} e^{-\mu_{a} z}
$$

and

$$
\mu_{a}=\frac{1}{\delta}
$$

where $z$ represents physical depth, $\mu_{a}$ is the optical absorption coefficient, $\Gamma$ is the Grunesian coefficient, $H_{0}$ is the incident laser energy per area, $\delta$ is the optical penetration depth, and $p_{0}$ is the photoacoustic pressure produced. The inverse of the optical absorption coefficient as shown in (4.5) is the optical penetration depth or

measure of the distance the light travels until it decays to $\frac{1}{e}$ of its initial optical intensity. Equation 4.4 with various experimental techniques are used to analyze the thickness, absorption, and imaging of many materials from blood vessels to thin films. $[58,115,120-123]$

\subsubsection{Total Internal Reflection Photoacoustic Spectroscopy}

When the refractive index of one medium is larger than that of a second medium, Snell's law, shows that beyond the critical angle, $\theta_{c}$, light is totally internally reflected back into the first medium given by

$$
n_{1} \sin \theta_{1}=n_{2} \sin \theta_{2}
$$

When $\theta \geq \theta_{c}$, and $\sin \theta_{2} \rightarrow 1$ 


$$
\theta_{c}=\sin \left(\frac{n_{2}}{n_{1}}\right)^{-1}
$$

This configuration produces total internal reflection, and generates a special type of nearfield optical effect called the evanescent field. The evanescent field can be perturbed to reduce the reflected coefficient of light if a sample material of appropriate optical absorption or scattering is sufficiently near the field. This type of frustrated total internal reflection is the basis of many characterization systems including attenuated total reflectance [101].

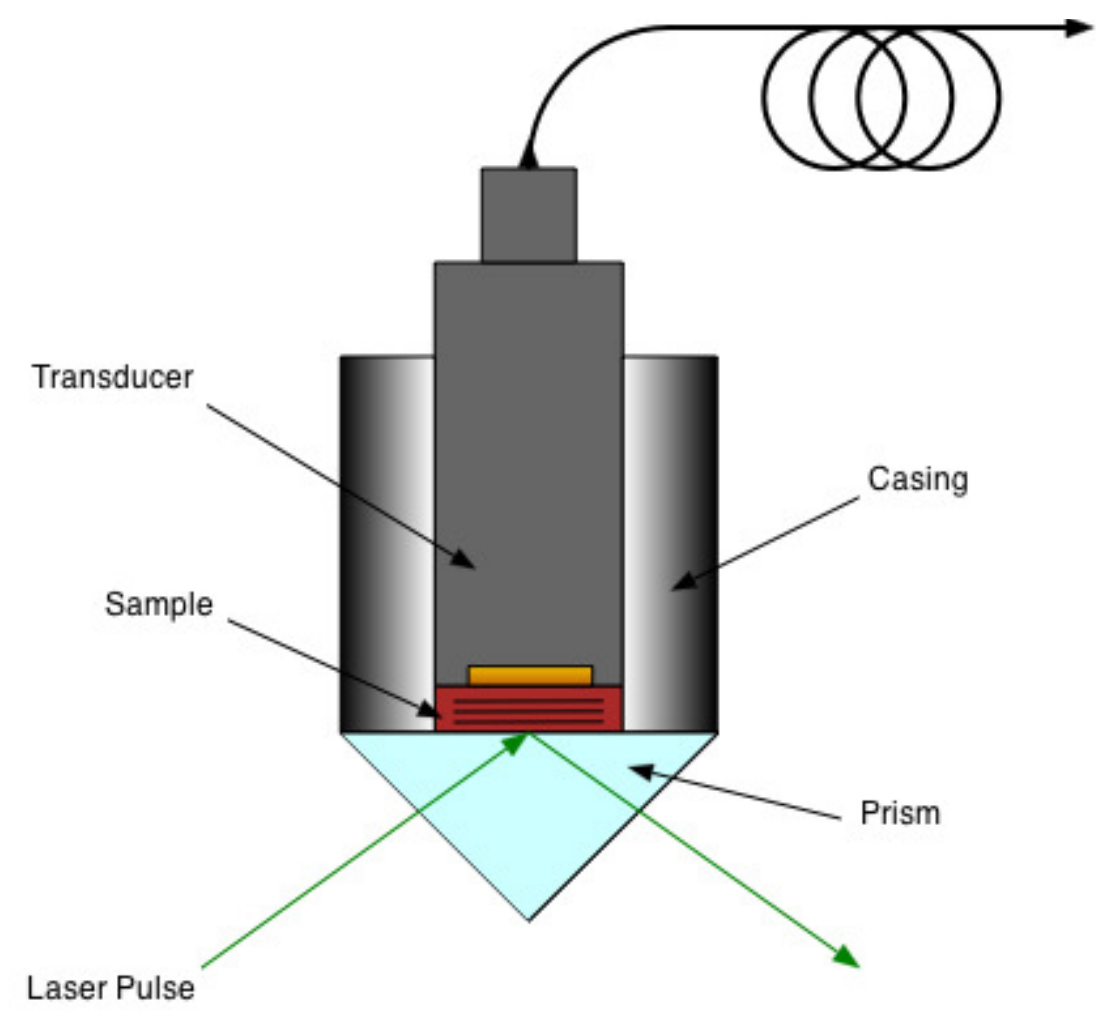

Figure 4.1: This figure shows a schematic of the setup showing how laser light interrogates sample to give an acoustic effect in TIRPAS.

Recently, a method of generating a photoacoustic response from optical absorption 
through a pulsed evanescent field was rediscovered by Goldschmidt et al. [88, 89], who used the technique in conjunction with modern lasers to improve the method's sensitivity. This technique is graphically demonstrated in Figure 4.1. The method has been used successfully to detect $\beta$-hematin to demonstrate the technique's potential as a new type of biosensor [89].

In the 1980 's, this technique [60-66,75-78] was explored as an alternative method to normal incidence photoacoustic spectroscopy, due to total internal reflection photoacoustic spectroscopy's (TIRPAS) ability to determine information about materials at an interface. This type of information was never achievable with normal incidence photoacoustic spectroscopy (PAS), due to the generally large optical penetration depth obtained with low absorption materials using the PAS method as can be seen in Figure 4.2. TIRPAS was used to determine dye adsorption properties at a glass/solution interface for interfacial phenomena studies as part of a cyclic voltammetry project.
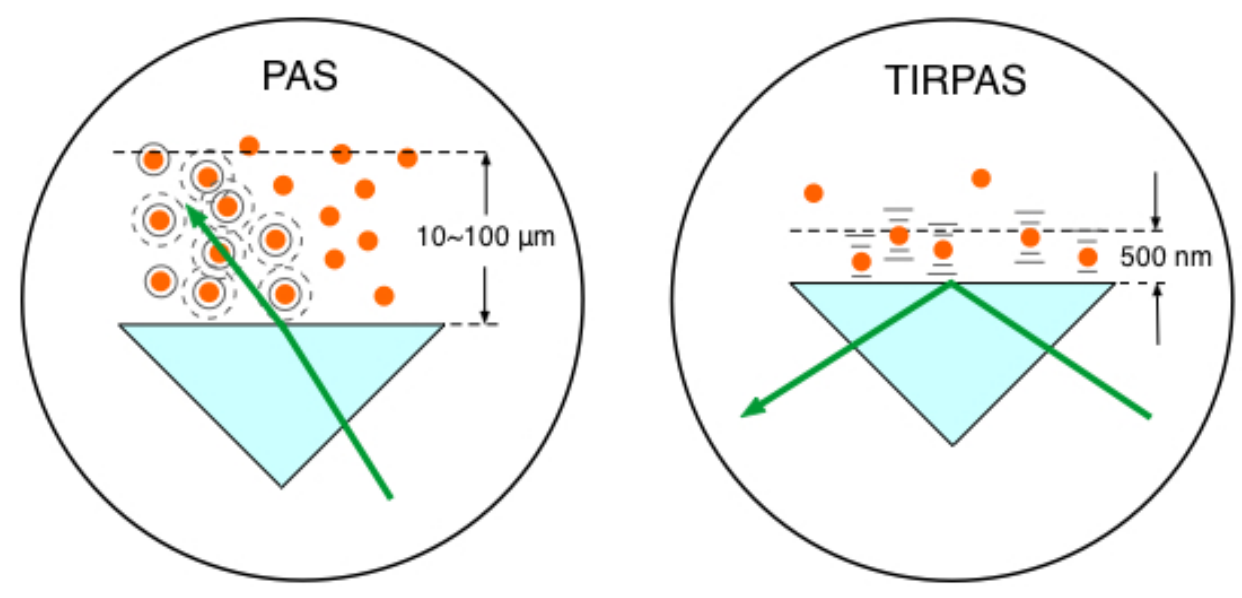

Figure 4.2: This figure illustrates the vastly different optical penetration depths between the PAS and TIRPAS excitation. 
The excitation of the photoacoustic effect using TIRPAS is fundamentally different than that by typical PAS experiments due to the fact that the evanescent field is interacting with absorbers close to the interface as opposed to the photoacoustic effect being excited by propagating photons through normal incidence. This type of excitation limits the optical penetration depth to the penetration of the evanescent field that is nearly always much less than the optical penetration depth of propagating photons through a typical material. The evanescent field component in polarization parallel to the optic is given by (4.7) that comes from (4.8) and (4.9), as presented by Muessig et al. [66] is given by

$$
\begin{gathered}
E_{\|}^{\prime}=\left(E_{x}^{\prime 2}+E_{z}^{\prime 2}\right)^{\frac{1}{2}}, \\
E_{x}^{\prime}=\frac{2 E_{\|}\left(\sin ^{2} \theta-n_{21}^{2}\right)^{\frac{1}{2}} \cos \theta}{\left(1-n_{21}^{2}\right)^{\frac{1}{2}}\left[\left(1+n_{21}\right) \sin ^{2} \theta-n_{21}^{2}\right]^{\frac{1}{2}}},
\end{gathered}
$$

and

$$
E_{z}^{\prime}=\frac{2 E_{\|} \sin \theta \cos \theta}{\left(1-n_{21}^{2}\right)^{\frac{1}{2}}\left[\left(1+n_{21}\right) \sin ^{2} \theta-n_{21}^{2}\right]^{\frac{1}{2}}},
$$

where $E_{\|}$is the component of the electric field parallel to the plane of incidence, $n_{21}=\frac{n_{2}}{n_{1}}, \theta$ is the angle of incidence of the laser on the prism/sample interface, and $E_{\|}^{\prime}$ is the parallel component of the evanescent field. Aside from the electric field components, which may affect the TIRPAS signal, the evanescent field's near field interaction can be described by a parameter called the optical penetration depth, as noted by Goldschmidt et al. [89]. The optical penetration depth shown in (4.10) describes the point where the non-propagating evanescent field decays to $\frac{1}{e}$ of its 
original intensity. This distance can be roughly used as the depth that can be analyzed with the photoacoustic effect in TIRPAS as given by

$$
\delta_{p}=\frac{\lambda_{1}}{2 \pi\left(\sin ^{2} \theta-n_{21}^{2}\right)^{\frac{1}{2}}},
$$

where $\lambda_{1}=\frac{\lambda}{n_{1}}, \lambda$ is the vacuum wavelength of the laser used and $\delta_{p}$ is the optical penetration depth of the evanescent field. This relationship is important because it implies that the signal magnitude in TIRPAS is proportional to the amount of material excited assuming the material is sufficiently thicker than the penetration depth of the evanescent field.

\subsubsection{Surface Plasmon Resonance Photoacoustic Spectroscopy}

Another method, related to TIRPAS, was explored in the early 1980s that used the resonant effect of surface plasmons to induce a photoacoustic effect for the detection of materials at an interface as well. Several groups [67-74,79-87] used this approach to understand the technique and to detect various materials, such as nicotine, and various gases of interest. The optical setups varied substantially, however, these methods generally used a laser pulse not meeting the excitation requirement for thermal confinement in the samples of interest. The entire systems were enclosed in photoacoustic cells for the detection and characterization of materials with properties related to and including refractive index. This technique, however, differs from TIRPAS in that TIRPAS uses pulsed laser light that interacts with a sample directly by the evanescent field. The related surface plasmon technique uses the generated evanescent field with an intervening metal layer to generate surface plasmons for detection 
of materials adsorbed on the surface of the metal layer.

\subsubsection{PAS/TIRPAS Refractometry}

PAS/TIRPAS refractometry is a new technique that combines the advantages of both the photoacoustic effect and the evanescent field to determine the refractive index of materials. It should be noted that a similar, but more computationally and physically complicated non-thermally confined photoacoustic refractometry technique was accomplished in the early 1990s. In that case, researchers relied upon the analysis of resonant optical modes in Langmuir-Blodgett films [124-127] and were able to produce refractive index estimates on the order of 0.01. In traditional reflectometry, light is measured after its interaction with a sample in two linear polarization states to determine refractive index. In PAS/TIRPAS refractometry, a laser beam is scanned across a sample at changing angles of incidence to collect acoustic waves from both the PAS and TIRPAS regimes, as well as any location inbetween. This experiment allows for the direct observation of the light-induced acoustic wave that changes drastically in peak to peak voltage between the PAS and TIRPAS regimes. This was shown experimentally in previous data from Goldschmidt et al. [89], and can be physically shown to be different by Figure 4.2. The drastic change in peak to peak voltage can be easily explained by comparing (4.5) and (4.4) with (4.10), which for typical values gives an order of magnitude or more difference in photoacoustic pressure. Here we demonstrate a simple, robust technique based upon the large changes in optical penetration depth for the determination of the real component of the refractive index of dyed liquids and the biologically relevant material, myoglobin, for potential disease detection. 


\subsection{Materials and Methods}

\subsubsection{Direct Red 81 Dye Solutions}

The dye solutions were made with a $50 \%$ polyethylene glycol (PEG, m.w. 200) 50\% $\mathrm{H}_{2} \mathrm{O}$ solution along with the dye at $125,250,500$, and $750 \mu \mathrm{g} / \mathrm{ml}$ respectively. The PEG was added in order to shift the refractive index closer to the middle range of the ATAGO R-5000 refractometer to provide easier measurement and comparison with the PAS/TIRPAS refractometer. Each solution was mixed for 5 minutes or until visually homogenous before testing using a hot plate/stirrer (Corning PC 220). The mixing directly preceeded each test to reduce the effects from dye settling on the prism surface.

\subsubsection{Myoglobin Solution}

The myoglobin solution was made by mixing $460 \mu \mathrm{g}$ of dry horse skeletal myoglobin (Sigma Aldrich \#M0630-250MG) per $1 \mathrm{ml}$ of phosphate buffered saline (Fisher \#BP2438-4). As with the dye solutions, the myoglobin was mixed for at least 5 minutes before testing using a hot plate/stirrer. The myoglobin solution did not have added PEG to it since the refractive index was within the readable scale on the handheld refractometer.

\subsubsection{PAS/TIRPAS Refractometer}

The device, shown in Figure 4.3, works on the principle of exciting a photoacoustic effect in the sample at different angles of incidence. The samples were interrogated 
with vertically polarized $532 \mathrm{~nm}$ laser light generated by a Q-switched Nd:YAG laser (Surelite I-20 Continuum). The acoustic signal was detected using a $15 \mathrm{MHz}$ transducer with a 1" focal length (Olympus \#U8472001).
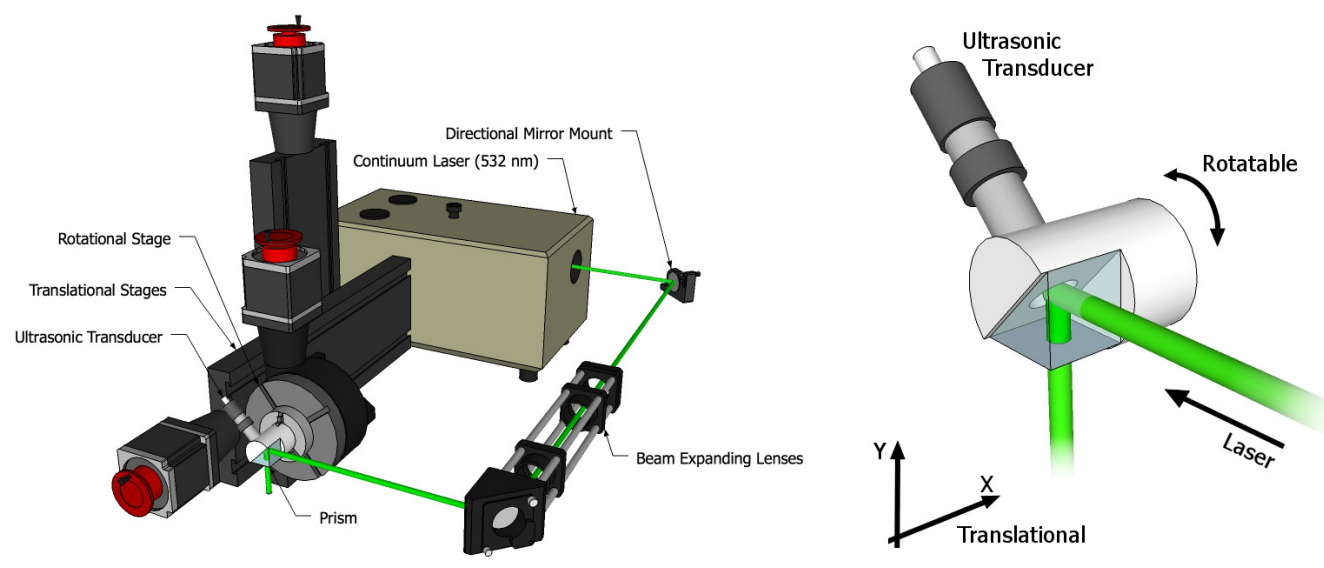

Figure 4.3: Left: This figure shows the full system including laser, stepper motor stage, and optical train. Right: This is a closeup of the PAS/TIRPAS refractometer sample holder used for testing.

\subsubsection{Laser Leveling}

The PAS/TIRPAS holder was leveled relative to the optical table by a Micromark digital level (Micromark \#: 84519). The interrogating laser beam was leveled relative to the table as well by a setup similar to a Michaelson interferometer except that the output of the setup was placed on a flourescent card where the two beams could be made coincident to level the beam. Thorlab's mirrors, a beam splitter, and cage system connectors were used to create this setup (Thorlabs \#: CM1-BS013 and \#: PF10-03-G01). The PAS/TIRPAS refractometer was aligned by viewing the incident and reflected spots on a sheet of fluorescent paper and adjusting the incident reflected 
laser to overlay it on that of the reflected beam.

\subsubsection{Labview Automation}

A Sherline stepper motor XY base (Sherline 5200-CNC) was used, along with a 4" rotary table $(\mathrm{P} / \mathrm{N} 8700)$, right angle attachment $(\mathrm{P} / \mathrm{N} 3701)$, and 3-jaw chuck $(\mathrm{P} / \mathrm{N}$ 1041) to hold and position the rotational mount made of a prism and custom machined PTFE rodstock. The rotary table was controlled by Labview. The angular spectrum settings were set to scan a range of angles where the critical angle was initially assumed to be. The Labview program stepped the motor at 0.01 degree increments across the entire range of angles. At each increment, the Labview program measured as stated the photoacoustic wave's peak to peak voltage.

\subsubsection{Exploratory Analysis}

The angular spectra were analyzed by taking the numerical derivative of the angular spectrum data to find the local minima corresponding to the reduction in photoacoustic signal amplitude. The process of taking the numerical derivative of the angular spectrum data produced visually noisy graphs that needed data smoothing to illustrate the existence of the critical angle. Kaleidagraph 4.1.0 was used with the smooth curve fit function to smooth the data so that the critical angle could be estimated from the data. This smooth curve fit is based upon a combination of Stineman inter-

polation [128] with a weighting structure to smooth the data. The exact weighting structure is described further in Appendix A.3. 


\subsubsection{PAS/TIRPAS Measurement}

First, the laser beam was leveled using the technique described in Section 4.2.4. Next, the sample holder was filled with sample liquid and the sample holder was leveled using the Micromark digital level by balancing the level on the unconnected acoustic sensor that was previously inserted into the sample holder. Finally, the Labview software described in Section 4.2.5 was used along with the Sherline stepper motors to obtain the angular spectrum scan of the sample material. The angular scan was then used to determine the critical angle as explained in Section 4.2.6.

\subsubsection{Optical Refractometer Measurement}

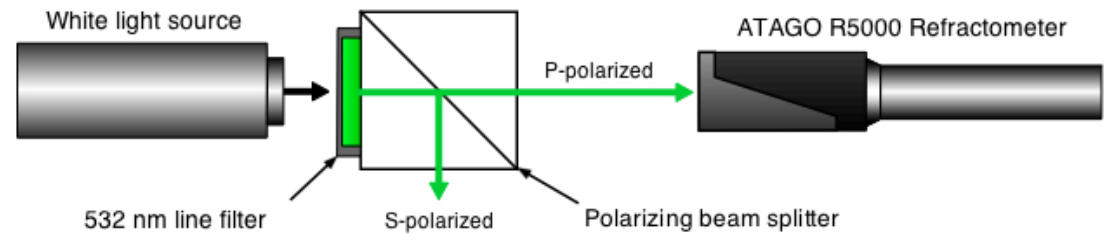

Figure 4.4: This figure shows the refractometer setup used to compare refractive index with PAS/TIRPAS refractometry. refractometry

We used an ATAGO R-5000 refractometer along with a $532 \mathrm{~nm}$ line filter (Thorlabs \#: FL532-10) and a polarizing beam splitter cube (Thorlabs \#: CM1-PBS251) to limit the polarization and wavelengths measured to find the refractive index of the samples for comparison with our PAS/TIRPAS refractometry technique as shown in Fig. 4.4. 


\subsection{Results}

A typical result from the dye is shown in Figure 4.5 and demonstrates a clear transition in photoacoustic peak to peak voltage at approximately 66 degrees. The numerical derivative of the angular spectrum data was taken along with smoothing to obtain a computable measurement of the transition as demonstrated by the arrow in Figure 4.5. Figure 4.6 shows a second smoothing applied to the data in order to obtain the minima corresponding to the critical angle. Finally, it is straightforward to determine the critical angle from finding the minima in Figure 4.6.
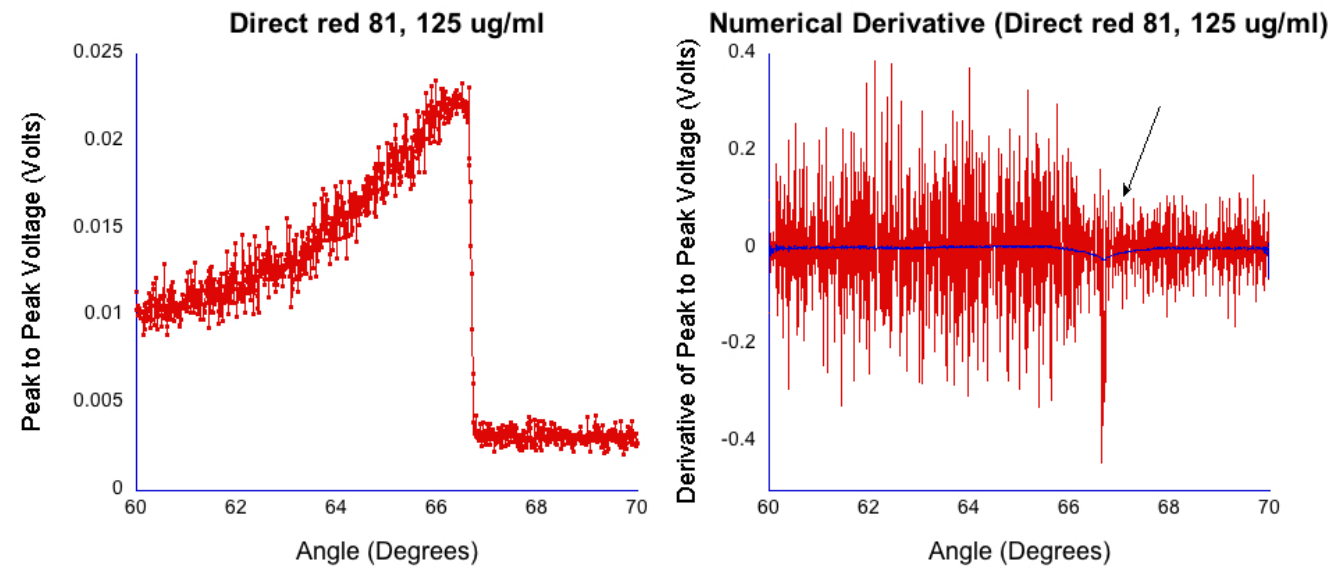

Figure 4.5: Left: This figure shows a typical angle spectrum (Direct red 81 at 125 $\mu \mathrm{g} / \mathrm{ml}$ ) that shows the characteristic drop [129] in peak to peak voltage from the PAS to TIRPAS regime. Right: This figure shows the numerical derivative of the figure on the left showing a smoothed Stineman function fit (Kaleidagraph 4.1) that displays a local minima corresponding to the critical angle.

In order to demonstrate the utility of PAS/TIRPAS refractometry, we compared the results obtained with optical reference measurements with the ATAGO R-5000 refractometer setup. We show illustrative agreement between the two techniques with the samples' refractive index values using PAS/TIRPAS refractometry and the 
ATAGO R-5000 refractometer as shown in Table 4.1. We took five measurements with PAS/TIRPAS refractometry and one measurement with the ATAGO R-5000 refractometer for comparison.
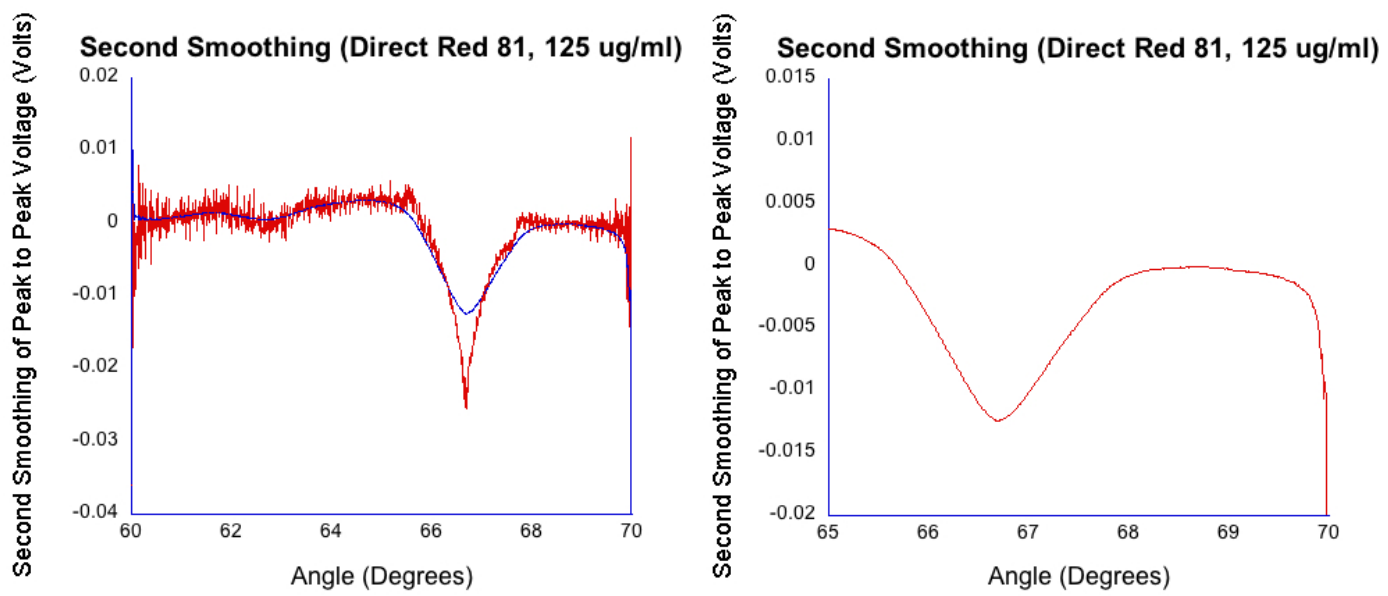

Figure 4.6: Left: This figure shows the second smoothing of Figure 4.5 that shows a second smoothed Stineman function fit (Kaleidagraph 4.1) that displays a local minima corresponding to the critical angle. Right: The second smoothed Stineman function (Kaleidagraph 4.1) displays a local minima corresponding to the critical angle.

\begin{tabular}{|c|c|c|c|c|c|c|}
\hline PAS/TIRPAS & Trial 1 & Trial 2 & Trial 3 & Trial 4 & Trial 5 & ATAGO R-5000 \\
\hline Direct Red $125 \mu \mathrm{g} / \mathrm{ml}$ & 1.395 & 1.395 & 1.395 & 1.395 & 1.395 & $1.395-1.397$ \\
\hline Direct Red $250 \mu \mathrm{g} / \mathrm{ml}$ & 1.390 & 1.390 & 1.390 & 1.390 & 1.390 & $1.390-1.396$ \\
\hline Direct Red $500 \mu \mathrm{g} / \mathrm{ml}$ & 1.388 & 1.389 & 1.389 & 1.389 & 1.389 & $1.381-1.395$ \\
\hline Direct Red $750 \mu \mathrm{g} / \mathrm{ml}$ & 1.382 & 1.382 & 1.387 & 1.387 & 1.387 & $1.372-1.395$ \\
\hline Myoglobin $460 \mu \mathrm{g} / \mathrm{ml}$ & 1.330 & 1.329 & 1.331 & 1.330 & 1.331 & 1.335 \\
\hline
\end{tabular}

Table 4.1: PAS/TIRPAS refractometry is compared to a handheld refractometer and demonstrates that our method is in good agreement with standard techniques. 


\subsection{Discussion}

As was illustrated in the previous section, PAS/TIRPAS refractometry seems to be a fairly accurate method that compares favorably to ellipsometry and reflectometry. Every dye solution's refractive index measurement fell within the measurement range of the handheld refractometer. In the case of the Direct Red, $750 \mu \mathrm{g} / \mathrm{ml}$, the sampleto-sample variability seemed to be more significant (0.005) than the other dyes. We believe this was due to dye settling over time. The myoglobin concentration seemed to have some divergence from the measured value with our handheld refractometer, however, this variability can be explained by the illustratively lower signal-to-noise ratio as shown in Figure 4.7. Of course, more research is needed to determine the error between these two conditions to determine quantitatively different values in signal to noise ratio. However, variability of 0.006 is still fairly reasonable compared to the differences between reflectometry and ellipsometry for many materials and therefore this is not substantial for our current demonstration of the technique's capabilities. Therefore, PAS/TIRPAS refractometry has the capability to analyze both dyes and a biologically relevant analyte, myoglobin, for potential biosensing and biomedical monitoring. Our technique opens up significant potential for biomedical monitoring and adsorption state monitoring through the refractive index at a sample/prism interface.

One interesting feature about PAS/TIRPAS refratometry is the generation of plane waves, which are acoustic waves, that deliver most of their energy in a forward and backward direction. The initial pressure rise due to these waves can be described by equation 4.4. In order to obtain plane waves in photoacoustics the following

equation must be satisfied: $z=\frac{d^{2} \mu_{a}}{8}$ where $d$ is the laser beam diameter and $\mathrm{z}$ is 

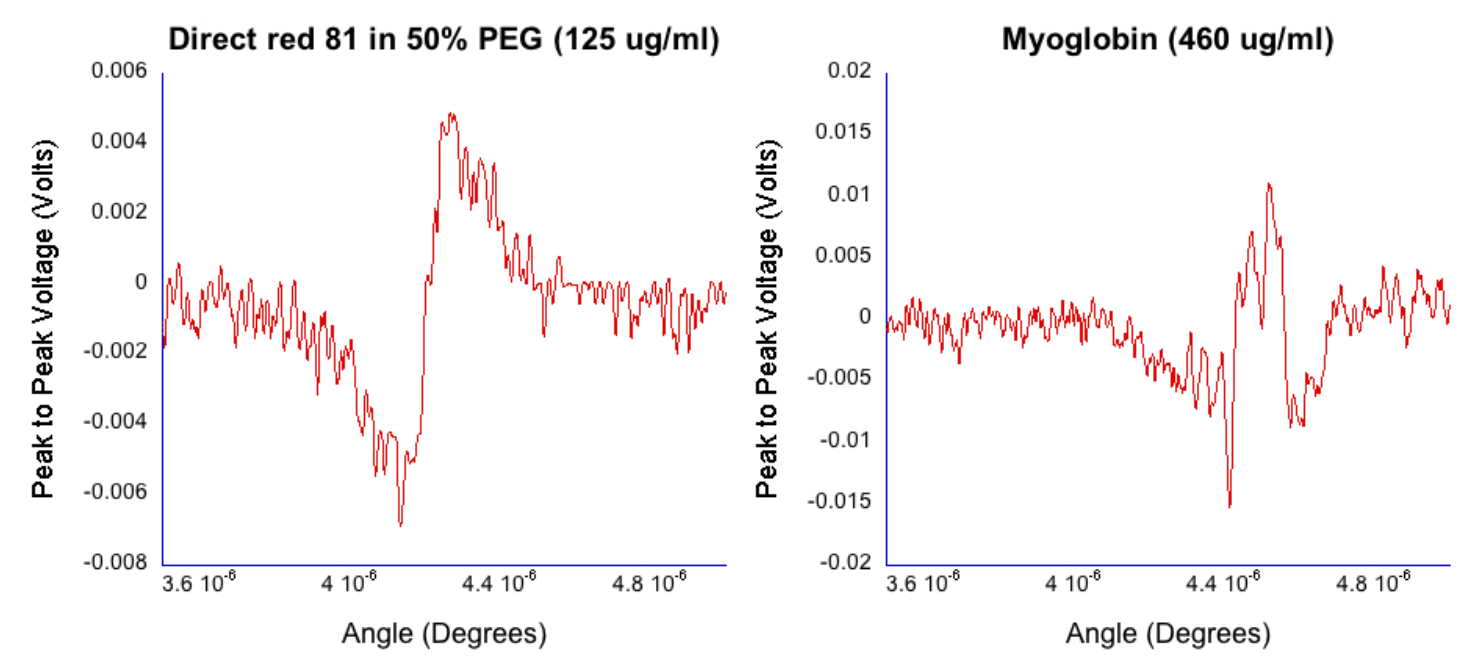

Figure 4.7: There are some acoustic differences between the dye solution and myoglobin solution under oblique photoacoustic excitation.

the acoustic near field/far field boundary as discussed by Viator [59]. In the PAS regime this is generally followed since the pigment of the dyes combined with the low optical penetration depth along with the geometry of the incoming beam create these conditions. However, with myoglobin, the solution is much more transparent and therefore, plane waves are not necessarily always obtained at every angle of incidence. In the TIRPAS regime, plane waves are always obtained due to the penetration depth of the optical radiation being so small compared to the relative size of the beam, 500 nm vs. $1 \mathrm{~mm}$ beam size or more. This can create issues when peak to peak voltage is compared in the PAS and TIRPAS regimes since it is possible for the TIRPAS regime to have similarly sized peak to peak voltage as the PAS regime, despite the PAS regime having more optical penetration depth due to the differences between comparing plane waves in the TIRPAS regime to non-plane waves in the PAS regime. Our data with myoglobin shown in Figure 4.7 suggest that this had an effect on our refractive index 
resolution since the acoustic waves from the PAS region with myoglobin do not show the typical plane wave configuration due to the low concentration of myoglobin used. An alternative method based upon integrated pressure may be more appropriate in the future.

Beyond the peak to peak differences, the two signals' relative shapes are worth mentioning. The myoglobin wave's complex shape comes from the fact that a cylindrical segment of sample is being excited that is giving rise to the acoustic wave. First, since angles of incident light smaller than the critical angle are used for the PAS regime, there is an effectively planar wave generated for both Direct Red and myoglobin when the refracted light transfers into the sample region at an angle close to 90 degrees from normal to the interface. This effect is due to the fact that the optical penetration depth is limited since the light is traveling nearly perpendicular to the sample/prism interface. However, at angles of incidence further away from the critical angle in the PAS regime, as shown in Figure 4.7, the myoglobin's signal starts to resemble a wave with more acoustic diffraction due to larger boundary waves being generated from the laser pulse incident at a lower angle of incidence from normal to the interface.

Table 4.2: This table shows a comparison of current technologies.

\begin{tabular}{|c|c|c|c|}
\hline & Resolution & Speed & Opaque materials? \\
\hline \hline PAS/TIRPAS refractometry & 0.006 & 54 seconds & Yes \\
\hline Ellipsometry & 0.001 & 1 second & No \\
\hline Reflectometry & 0.001 & 1 second & No \\
\hline Hand held refractometer & 0.001 & 1 second & Yes \\
\hline
\end{tabular}




\subsection{Conclusion}

As can be seen from Table 4.2, PAS/TIRPAS refractometry should have several uses for materials that are heavily opaque with unknown optical properties. This suggests the use of PAS/TIRPAS refractometry with metals as well as less homogenous materials for quick and simple critical angle analysis. PAS/TIRPAS refractometry has two major advantages over current methods that include the ability to analyze deeply opaque materials along with a true single wavelength determination of the refractive index without the need for complex model fitting needed for other techniques.

PAS/TIRPAS refractometry has shown promise in its ability to determine refractive index values for myoglobin at a diagnostically relevant concentration. This opens the technique to biological monitoring of myoglobin levels before and after surgery to aid doctors in the diagnosis of potential renal problems occurring from the accumulation of myoglobin after acute hyperthermia or myocardial infarction. In addition, PAS/TIRPAS refractometry, if combined with an automated syringe pump, could be used for the monitoring of levels of important biological analytes in blood, urine, and for the determination of binding kinetics similar to surface plasmon resonance.

We have demonstrated a new photoacoustic technique that can be used in place of ellipsometry and optical reflectometry for the determination of the real part of the refractive index of liquid solutions. Additionally, we have demonstrated the refractometer's capability to determine the refractive index of myoglobin at a diagnostically

relevant concentration. This new technology has been shown to complement standard technologies such as ellipsometry and reflectometry for future biomedical testing. 


\section{Chapter 5}

\section{QTPAS and Thin Films}

Thin films of sol-gel or mesoporous silicas for optoelectronic use currently require several types of analytical instruments for proper characterization of crucial parameters, such as refractive index, porosity, thickness, and homogeneity. Currently, spectroscopic ellipsometry is the standard method for determining refractive index of such films; however, this technique has difficulty analyzing non-reflective materials, such as structured or porous films of micrometer or larger thickness. Moreover, ellipsometry produces values for refractive index that are dependent on thickness and knowledge of the film's substrate or other layers it is packaged with. Here we present an alternative method, using the photoacoustic effect, to non-destructively determine the refractive index of thin films independently of thickness using well-understood mesoporous silica thin film systems as a proof of concept. 


\subsection{Photoacoustic Evaluation of Thin Films}

There is a substantial amount of existing literature regarding using the photoacoustic effect to evaluate surfaces and thin films $[130,131]$ for a variety of physical parameters. Types of parameters studied with this configuration include spectroscopic evaluation, thickness or depth profiling evaluation, and even thermal diffusivity and even thermal conductivity could be studied for particular films [132]. Spectroscopic evaluation is simple to employ on standard substrates such as deposited films on silicon since optical methods $[102,133-135]$ exist for the detection of acoustic waves in a variety of configurations. The primary limitation with spectroscopic evaluation of thin films with the photoacoustic effect is to scan a material with effectively zero absorption over the optical wavelengths studied. With many mesoporous silica materials, there are very few absorption bands even into the near-IR portions of the spectrum compared to other materials. This optical transparency limits traditional photoacoustic evaluation of these materials for any properties unless a mid-IR laser or mesopore introduced chemical can be used for absorptive contrast [136] of the films.

\subsection{Quantum Tunneling Photoacoustic Spectroscopy}

Quantum Tunneling Photoacoustic Spectroscopy (QTPAS), is a technique that uses the photoacoustic effect coupled with optical tunneling to determine thickness and refractive index of nanofilms. QTPAS works on the same principle to the classical optical tunneling experiment where light can traverse a small gap through interaction

of a high refractive index material with the extending evanescent field created through total internal reflection. 


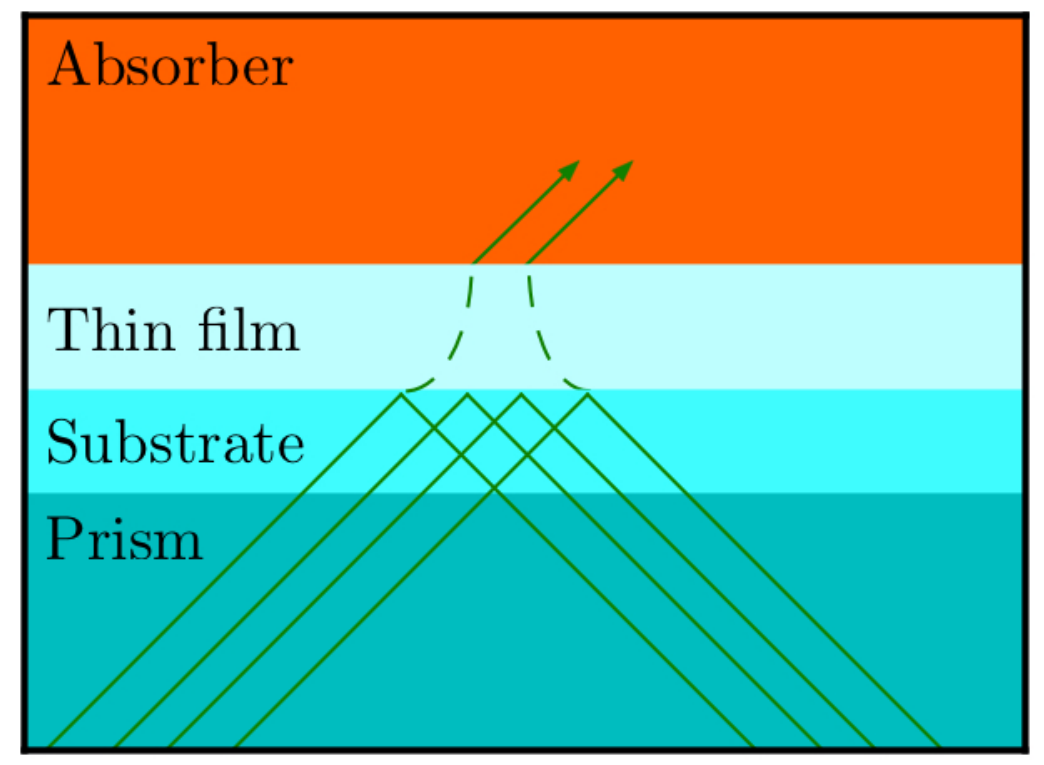

Figure 5.1: Quantum tunneling photoacoustic spectroscopy can be described by a schematic of four layers of material with laser interaction through them as shown above.

Figure 5.1 shows that light becomes delocalized and can "tunnel" through a thin film when the light is under total internal reflection. Equations [137] to describe optical tunneling with $\mathrm{S}$ and $\mathrm{P}$ polarization states for light are shown below as

$$
T=\left(\alpha \sinh ^{2} y+\beta\right)^{-1},
$$

which describes what percentage of light will cross the thin film.

$$
y=2 \pi \frac{n_{1} d}{\lambda} \sqrt{N^{2} \sin ^{2} \phi_{0}-1}
$$

Equation (5.2) describes the optical penetration depth of the evanescent field into the film of interest. 


$$
\begin{gathered}
\alpha_{s}=\frac{\left(N^{2}-1\right)\left(n^{2} N^{2}-1\right)}{4 N^{2} \cos \phi_{0}\left(N^{2} \sin ^{2} \phi_{0}-1\right) \sqrt{n^{2}-\sin ^{2} \phi_{0}}} \\
\beta_{s}=\frac{\left(\sqrt{n^{2}-\sin ^{2} \phi_{0}}+\cos \phi_{0}\right)^{2}}{4 \cos \phi_{0} \sqrt{n^{2}-\sin ^{2} \phi_{0}}}
\end{gathered}
$$

Equations (5.3) and (5.4) refer to the attenuation constant and the propagation constant of the system assuming S polarized light.

$$
\begin{gathered}
\alpha_{p}=\frac{\alpha_{s}}{n^{2}}\left\{\left(N^{2}+1\right) \sin ^{2} \phi_{0}-1\right\}\left\{\left(n^{2} N^{2}+1\right) \sin ^{2} \phi_{0}-n^{2}\right\} \\
\beta_{p}=\frac{\left(\sqrt{n^{2}-\sin ^{2} \phi_{0}}+n^{2} \cos \phi_{0}\right)^{2}}{4 n^{2} \cos \phi_{0} \sqrt{n^{2}-\sin ^{2} \phi_{0}}}
\end{gathered}
$$

Equations (5.5) and (5.6) refer to the attenuation constant and the propagation constant of the system assuming $\mathrm{P}$ polarized light where $n=\frac{n_{2}}{n_{0}}$ and $N=\frac{n_{0}}{n_{1}}$ These equations describe how the light tunnels through a thin film of interest based upon different film/substrate properties. In Figure 5.2, it can be seen that differences in thickness of the film with a given refractive index (1.4) affect the quantum tunneling by shifting the transmission curves lower with higher inflection as the thickness of the film increases. This makes sense because the thicker the film becomes, the less the evanescent field can penetrate into a guiding material thus leading to less optical tunneling. Additionally, it can be seen in Figure 5.2 that increases in the refractive index of a film with given thickness $(300 \mathrm{~nm})$ tend to shift the critical angle closer to 90 degrees but more or less keep the shape of the spectrum intact unless the refractive index becomes very close to the value of the substrate. 

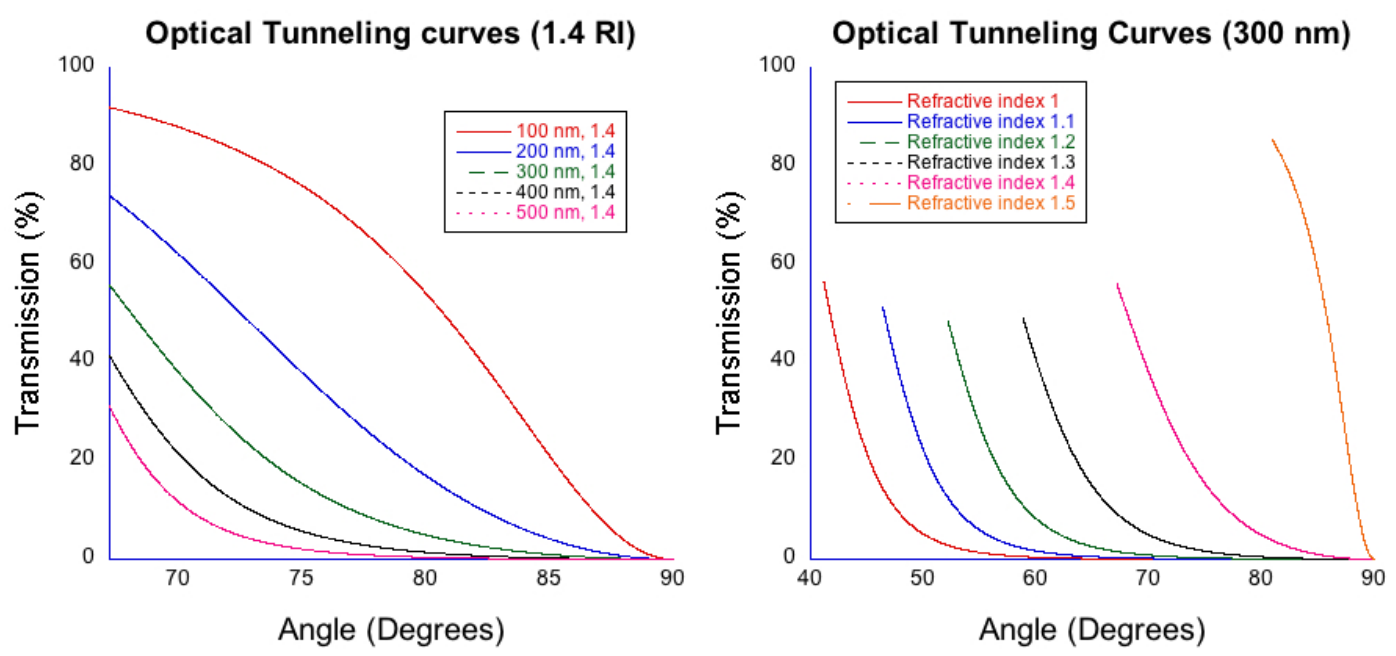

Figure 5.2: Left: Optical tunneling transmission curves for different film thicknesses show that thicker films reflect more light than thinner films of the same refractive index. Right: Optical tunneling for different refractive indices shows that higher refractive indices shift the curves to higher angles

\subsubsection{QTPAS Derivation}

The transmission of light through a film due to quantum tunneling is insufficient to completely describe the inner workings of QTPAS since there is of course a photoacoustic component to it and not just optical. The photoacoustic effect's working principle is that sound is generated through localized transient optical absorption of light. According to [137] we define (5.1) at zero film thickness (Fresnel reflection) as

$$
T_{0}=\beta^{-1} \text {. }
$$

Therefore, we can introduce a conversion factor, $K$, that can describe the conversion of light absorbed to acoustic transmission. Therefore, we can create (5.8) to explain how acoustic transmission in volts, $P$, can be transformed into normalized transmission 
from 0 to $1, T . K$ is a function of the sensitivity of the transducer, the optical absorption of the acoustic emitter, and weakly on the temperature of the material being irradiated.

$$
\frac{T}{T_{0}}=\frac{p K}{p_{0} K}
$$

If we use (5.8) and set it equal to (5.1) and (5.7) we can write

$$
\frac{T}{T_{0}}=\frac{p K}{p_{0} K}=\frac{\left(\alpha \sinh ^{2} y+\beta\right)^{-1}}{(\beta)^{-1}} .
$$

With a little cancellation and rearranging we have

$$
T=\frac{p}{p_{0} \beta}=\left(\alpha \sinh ^{2} y+\beta\right)^{-1} .
$$

This fully reduces to

$$
P^{\prime} \beta^{-1}=\left(\alpha \sinh ^{2} y+\beta\right)^{-1},
$$

where $P^{\prime}$ is the normalized acoustic voltage obtained by scanning a substrate with thin film divided by a blank substrate. In the case of matching refractive index between the prism and the acoustic emitter $\beta=1$ and the equation can be simplified further to

$$
P^{\prime}=\left(\alpha \sinh ^{2} y\right)^{-1}
$$




\subsubsection{Assumptions/Limitations of the QTPAS Derivation}

There are several assumptions that must be mentioned before the QTPAS equations, (5.9) and (5.10), can be applied to a real situation.

1. Temperature of acoustic emitter is constant

2. Laser energy per pulse and distribution is constant

3. Refractive index of prism, substrate, thin film, and acoustic emitter are constant

4. Optical absorption into acoustic emitter is constant

5. Optical coupling must be constant between scans

6. Optically transparent thin film

7. Film thinner than $1500 \mathrm{~nm}$

With regard to 1 , the temperature of the acoustic emitter needs to be relatively constant because the photoacoustic effect will increase or decrease depending upon the temperature of the absorber. This can be shown to describe photoacoustic plane waves [107] and (5.11) as

$$
p(z, t)=\frac{1}{2} \Gamma H_{0} \mu_{a} e^{-\mu_{a}(z-c t)}+\frac{1}{2} \Gamma H_{0} \mu_{a} e^{-\mu_{a}(z+c t)}
$$

and

$$
\Gamma=\frac{\beta}{\kappa \rho C_{v}},
$$


where $\beta$ is the thermal coefficient of volume expansion, $\kappa$ is the isothermal compressibility, $\rho$ is the density, and $C_{v}$ is the specific heat capacity at constant volume. Since $\beta, \kappa, \rho$, and $C_{v}$ are functions of temperature $\Gamma$ also varies with temperature. Although experimentally it is important to keep the acoustic emitter at a constant temperature, this is truly difficult since a laser is constantly impinging upon the surface causing acoustic waves to be emitted. Luckily, steady state heating is achieved with a few laser pulses and therefore no overall correction is needed for practical testing.

In addition to constant temperature, the laser energy's distribution (Gaussian) and pulse energy needs to be held constant in order to compare the light transmission from one pulse to the next. Unfortunately, Nd:YAG lasers are very poor lasers for optical stability since at specifications energy fluctuation may be as high as +$3.5 \%$. Since the photoacoustic effect works by linear thermoelastic expansion this suggests that a $+-3.5 \%$ fluctuation in laser energy would lead to a $+-3.5 \%$ fluctuation in acoustic signal that, due to the normalization, $P^{\prime}$, would create an even higher error in the overall measured transmission. To correct for this fluctuation in laser energy, the QTPAS system performs a dual photoacoustic measurement to measure not only the voltage from the transducer coupled to the substrate/film system, but additionally to a transducer with the same acoustic emitter with the laser light normally incident into it. The additional transducer/acoustic emitter system provides the function of measuring the "laser energy" and allowing for the photoacoustic signal to be normalized based upon small fluctuations in laser energy. Additionally, since both transducers are measuring laser light incident upon the same type of acoustic emitter, the fluctuations from changes in temperature based upon the Grunesian coefficient are approximately cancelled out since both materials heat similarly. 
With regard to 3 , we must make the assumption that the refractive indices of the thin film, substrate, prism, and acoustic emitter are constant. If the refractive indices are not constant, the properties of both refractive index and thickness (since they are interdependent) will change based upon (5.2)-(5.6). Changes in temperature as well as the presence of an acoustic wave can change the refractive index of these materials. However, generally temperature only needs to be taken into account when doing a refractive index measurement when precision beyond 0.001 refractive index units is needed that, in the case of our preliminary study, is not the case. Additionally, the refractive index of these materials will only change during the presence of an acoustic wave which, since there are no interfering acoustic waves until after the light has interacted with the materials, the assumption of no acoustic interference still holds, however, this no interference assumption means that previous iterations of photo acoustics (where a Hemholtz cavity is used to increase signal to noise ratio) will not work due to the temporal and spatial limitations of the system.

With regard to 4 , we make the assumption that the optical absorption of the acoustic emitter stays constant. This assumption means that we cannot excite the acoustic emitter so much that it starts to chemically change or burn. If the material burns or changes chemical properties dramatically, this can affect the signal by changing the emitter's acoustic impedance and by changing the emitter's optical absorption (typically burned materials turn black and so the photoacoustic signal increases). In order to prevent this effect, the laser energy must simply be lowered so that burning or chemical changes do not occur.

With regard to 5 , it is important to keep the optical coupling the same from one scan to the next (particularly from the $p_{0}$ scan to the $p$ scan). The reason is that if the 
optical coupling is not the same from one test to the next, there may be nanoscopic air gaps in-between the film and the acoustic emitter. If there are gaps then the overall thickness and refractive index will be incorrect since the system will calculate them based upon the combination air gap/thin film.

With regard to 6 , the films being interrogated need to be transparent. The reason for this is that the optical tunneling equations given in [137] do not take into account absorption due to the evanescent field interacting with absorbers in the film. Since the evanescent field has to exist in the film for optical tunneling to occur, any optical absorption within the film will change the overall measurement of the tunneling in the resulting acoustic wave and thus change the tunneling measurement.

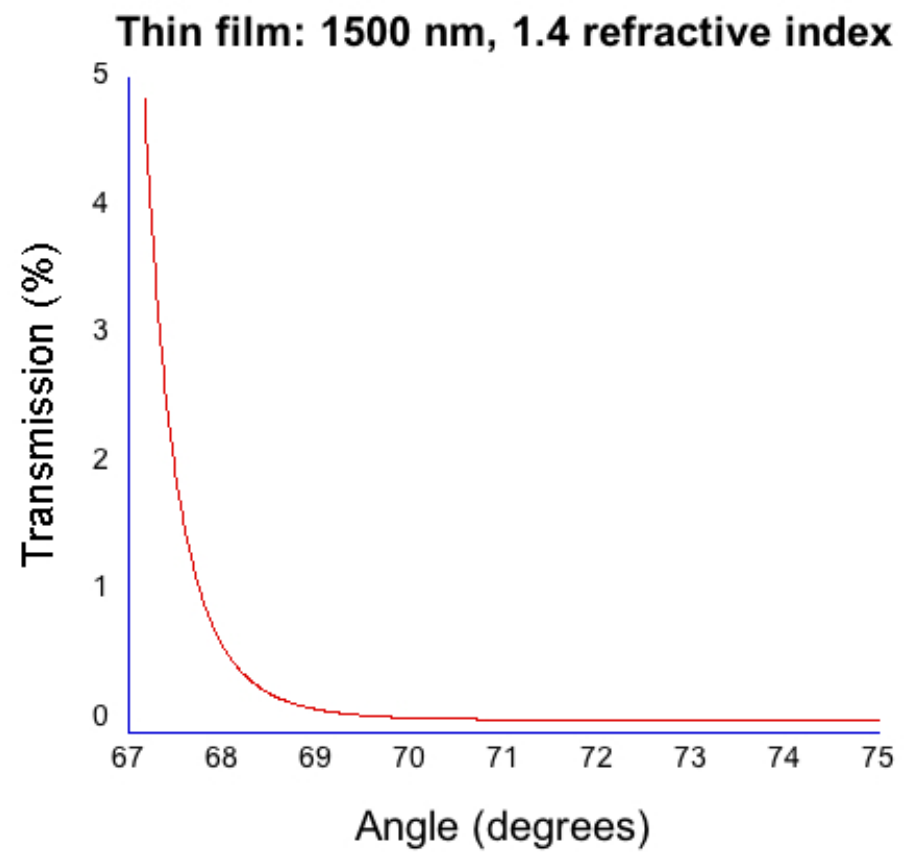

Figure 5.3: Optical transmission is shown for a $1500 \mathrm{~nm}$ film. 
Finally, with regard to 7 , it is important that the thin film under investigation be thinner than approximately $1500 \mathrm{~nm}$ in size. The reason for this is that as the thickness of the film is increased more reflection occurs beyond the critical angle through optical tunneling. Although the probability of finding a tunneled photon is not truly ever zero (since the probability decays exponentially from the interface), there is a physical limitation of being able to measure whether or not a photon tunnels far away from the interface due to the fact that to get a measurable photoacoustic signal there must be at least several hundreds of thousands of photons exciting the acoustic emitter to generate a measurable sound. In the current system it is difficult to determine changes much smaller than $5 \%$ transmission. Therefore, if we use $5 \%$ as our cutoff we can calculate the tunneling profile of an example film (1.4 RI, 1500 um) and show that this film only tunnels approximately $5 \%$ of the initial light at the extreme value near the critical angle as shown in Figure 5.3.

\subsection{Labview Automation: QTPAS.vi}

In order to run the QTPAS system it became necessary to fully automate the rotation of the prism/sample interface as well as to automatically collect signals through a digital acquisition system. A schematic of that setup is shown in Figure 5.4. Beyond the physical setup, a Labview program was created to run the system called "QTPAS.vi". Figure A.8 shows that the program has three main modes that can be user selected, Setup, Angular Spectrum, and Curve fitting.

- Setup: Allows the user to see signals at a single angle of incidence to confirm correct system operation 
- Angular spectrum: Allows the user to run an "angular spectrum" test for later evaluation under TIRPAS, PAS/TIRPAS refractometry, or QTPAS analysis techniques

- Curve fitting: Allows the user to fit a function to collected data using (5.9) to estimate $n_{1}$ and thickness of thin films using an exploratory method to get a general range of the properties.

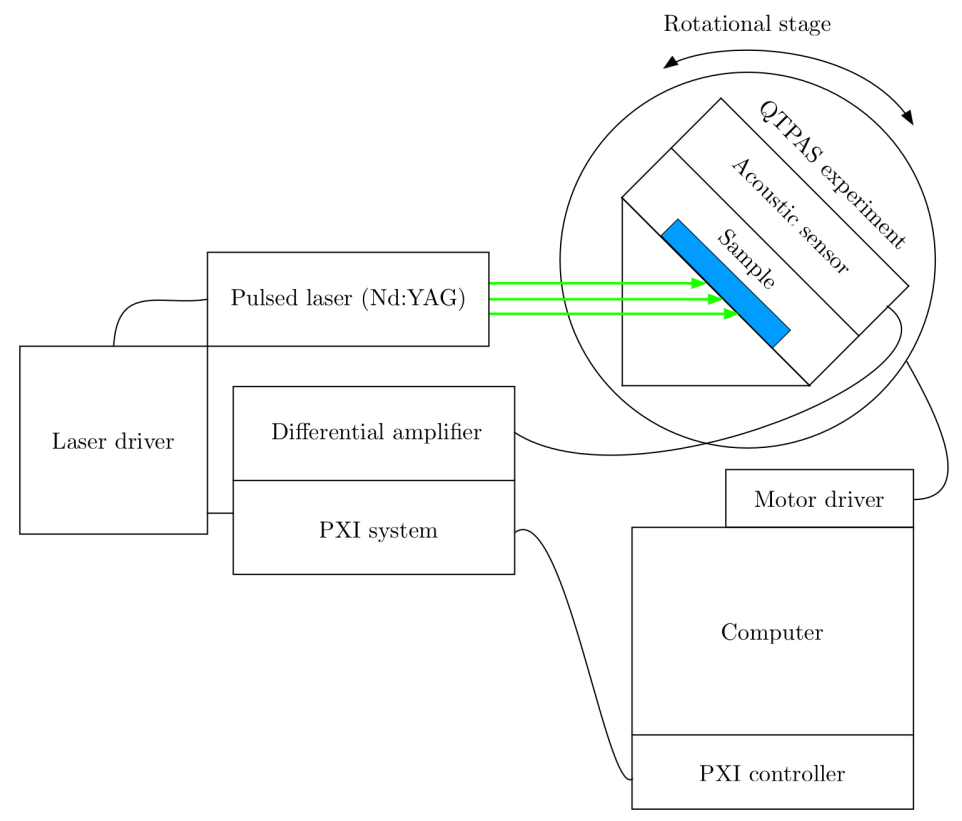

Figure 5.4: QTPAS schematic shows how electronics and physical components fit together.

Beyond selecting the modes, the user must input parameters such as the refractive index of the surrounding air, the prism, the acoustic emitter, and set the band pass signal filter boundaries, etc. Once the initial parameters and defined cursor regions are set, the user can run the program to get the desired result of setup running, an 
angular spectrum scan, or can perform a non-linear least squares curve fit to (5.9) to estimate the parameters $n_{1}$ and thickness using this exploratory method.

\subsubsection{Refractive Index and Thickness Estimation}

Initial estimation of refractive index and thickness is accomplished by using an unweighted constrained non-linear curve fitting algorithm developed by National Instruments. The method is the Levenberg-Marquardt algorithm [138] that is used to find a local minima given an initial guess for thickness and refractive index based upon fitting to equations describing light transmission given in [137] for $\mathrm{S}$ and $\mathrm{P}$ polarization. Specifically, (5.1) is the equation that is fit to for determining the refractive index and thickness for QTPAS material property estimation. Since (5.9) only describes optical tunneling given the total internal reflection assumption it is important

to use the constrained non-linear curve fit. The author originally tried using the non-constrained non-linear curve fitting algorithm for simplicity, however, depending upon the initial guesses it was possible for the algorithm to guess values of $n_{1}$ and thickness that would give rise to imaginary numbers that could cause an error in the virtual instrument. More information about the non-linear curve fit can be found at: http://zone.ni.com/reference/en-XX/help/371361G-01/gmath/nonlinear_ curve_fit/\#parent

\subsection{Exploratory Analysis and Simulated Example}

Although QTPAS has not yet demonstrated an accurate evaluation of the thin film properties, thickness and refractive index, I have included experimental data as well as 
a simulation that qualitatively explores the current state of the art in the developing field of QTPAS. Simulated data such as Figure 5.5 was created using Matlab code to add random noise to a function and is found in Appendix A.6. In the simulation the standard deviation or (sigma $)=10$ and mean or (Baseline $)=1$. This simulation provides an illustration of a typical signal.

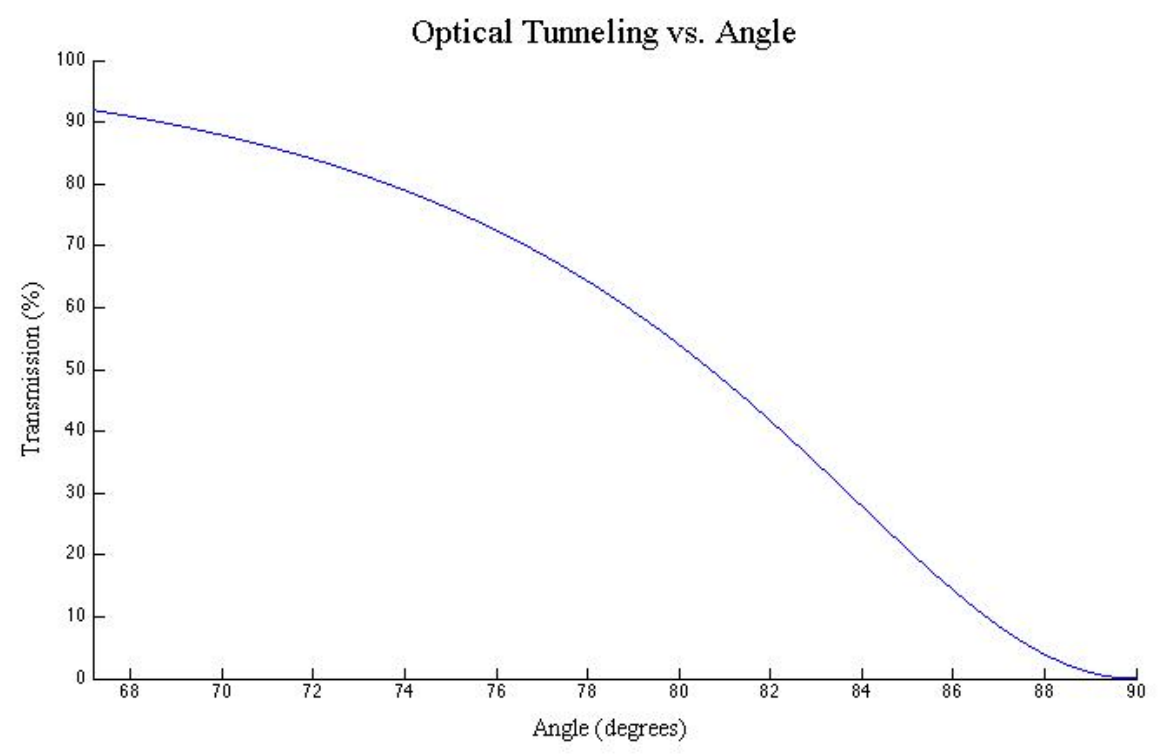

Figure 5.5: Optical transmission based upon $n_{1}=1.4$ and $100 \mathrm{~nm}$ thickness shows substantial change in light transmission over a range of angles of incidence.

\subsubsection{Substrate Scans}

In order to calibrate the QTPAS system one must scan a substrate (NBK-7 glass) and then scan another substrate with the film on it to divide one scan by another and apply (5.12). Since the substrate and acoustic emitter are the same refractive index, $\beta=1$, so the equation can be simplified to 


$$
P^{\prime}=\frac{p}{p_{0}}=\left(\alpha \sinh ^{2} y\right)^{-1}=1
$$

Ideally, the equation reduces to equal 1 because the same substrate is being scanned twice and therefore, the same acoustic effects should be obtained over the same scanning angles.

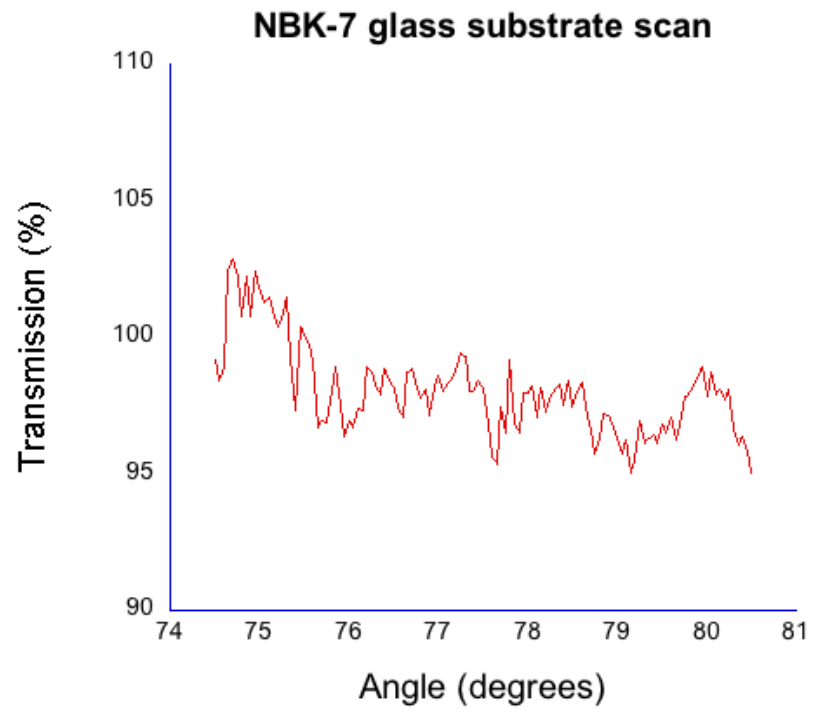

Figure 5.6: This figure shows a representative scan of a substrate divided by itself.

In practice, the graph obtained from scanning the same substrate twice can be represented by Figure 5.6. To show that this is the case, a simulation with the introduction of Gaussian white noise to a predicted optical tunneling curve for a film $n_{1}=1.4$ of $100 \mathrm{~nm}$ thickness can be seen in Figure 5.7. In order to reduce the Gaussian noise inherent with all electronic systems several techniques have been employed with the QTPAS system. These techniques include averaging waveforms together at each angle of incidence 128 times as well as to use a bandpass Chebyshev 5th order infinite impulse response filter with a range from $1 \mathrm{~Hz}-5 \mathrm{MHz}$ to reduce noise. 


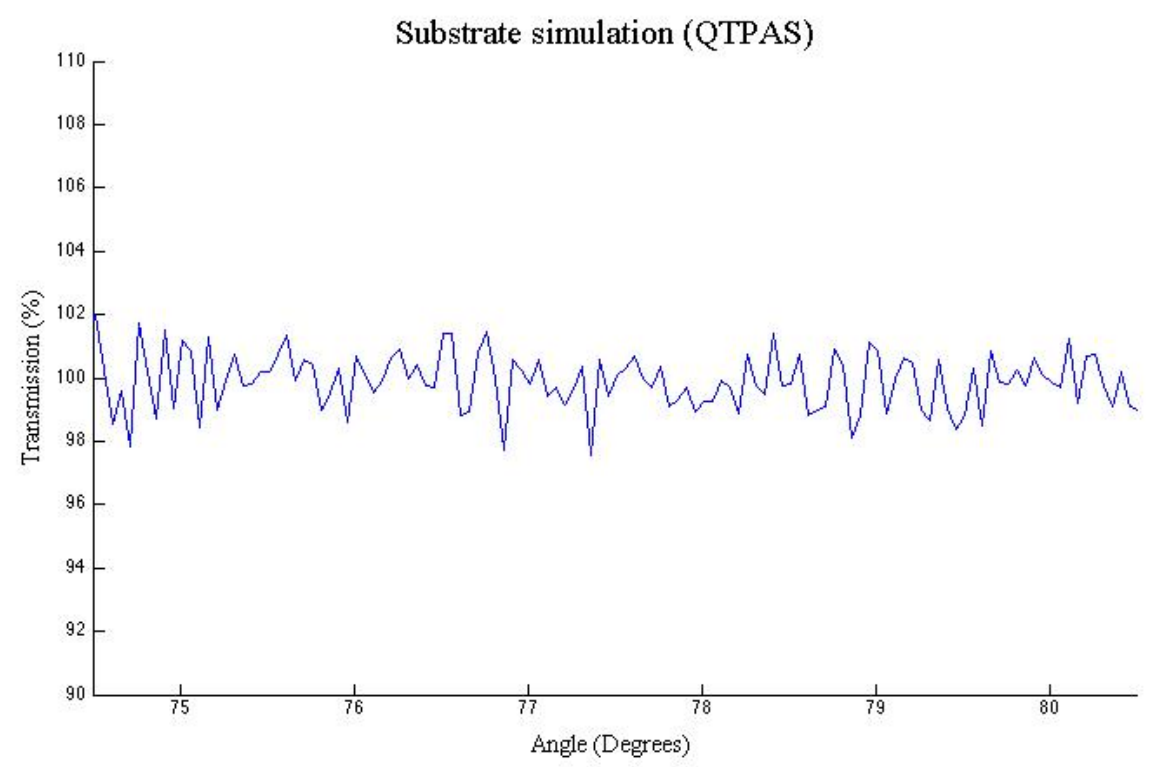

Figure 5.7: This figure shows a simulated data of a substrate scan with Gaussian white noise.

\subsubsection{Laser Energy Interaction}

Interestingly enough, the most recent testing with QTPAS indicates that the results obtained from an angular spectrum depend upon the laser's energy intensity per pulse. In Figure 5.8 a representative sample shows that the slope of the angular spectrum graph seems to be qualitatively different when high energy (Q-200) and low energy (Q-270) are compared. Of course, more testing is needed to conclude this with any statistical significance, however, with a hypothetical understanding of this effect one would think that this should not be occurring since energy differences are accounted for by measuring the relative laser energy at every pulse. However, since the two curves seem to be different this suggests that there may be some interaction between the laser beam's intensity and the resulting acoustic wave obtained for the scan. In order to determine what possible effects may occur over time angular spectra were 
taken on the same substrate over a seven hour period of time to get an idea of how the spectra would change as shown in Figure 5.8.

As can be seen from the spectra, the curves seem to increase in peak to peak voltage with every additional test bringing up the possibility that overall sample heating may be occurring that would affect the refractive index and Grunesian coefficient of the sample or some complex form of photobleaching may be occurring that may increase the efficiency of photoacoustic generation.

High Energy vs. Low Energy
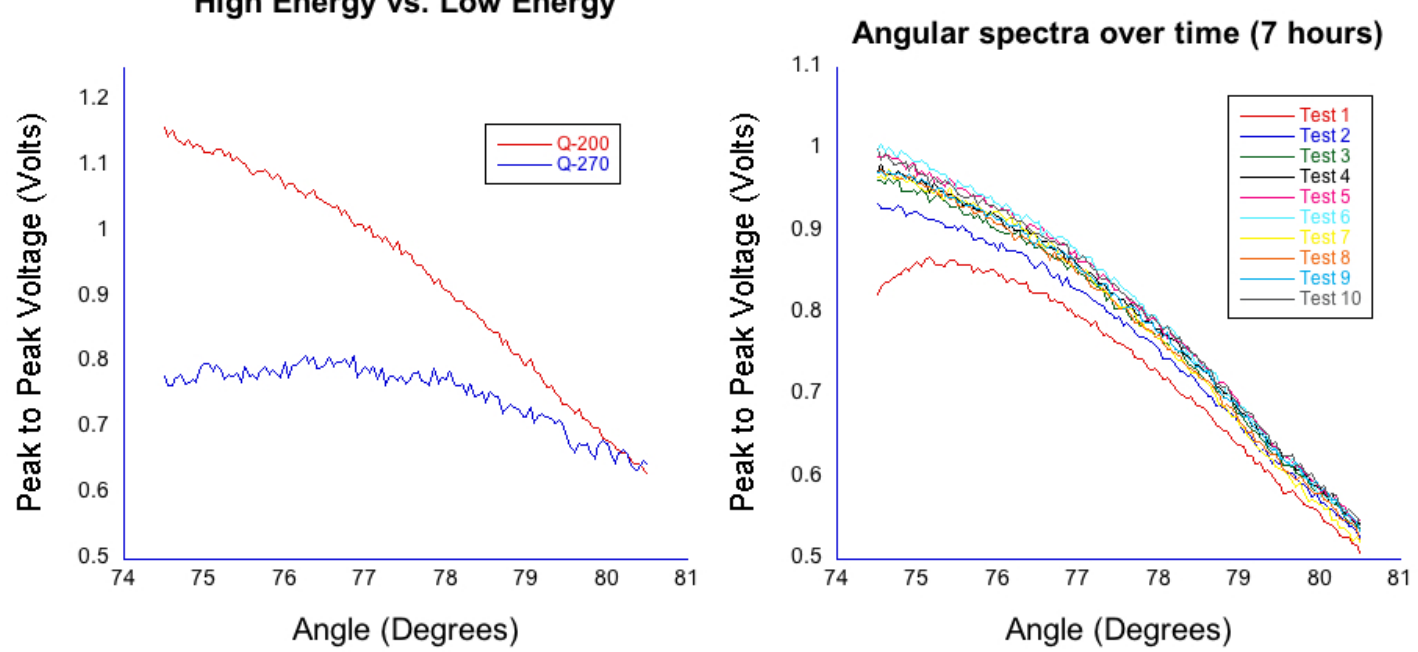

Figure 5.8: Left: Differences in angular spectra shape can be seen for two laser qswitch values for the same substrate. Right: This figure shows angular spectra taken over a seven hour period to illustrate changes in spectra over time.

There is ongoing research to investigate this effect to determine what the effect is from and how strongly it affects the resulting data. 


\section{Chapter 6}

\section{Future Work}

Due to time constraints it was not possible to explore all possible avenues for research regarding TIRPAS, QTPAS, and related techniques. However, a review of the possibilities may be helpful for future researchers to explore new technologies and techniques.

\subsection{Birefringence Determination}

Provided is a schematic drawing of a method to simultaneously determine the refractive index of a material for both $\mathrm{S}$ and $\mathrm{P}$ polarizations. The incident beam from the

laser is passed through a $\frac{\lambda}{2}$ wave plate to rotate the polarization of the incident beam to split the beam into two components ( $\mathrm{S}$ and $\mathrm{P}$ polarized). The $\mathrm{P}$ polarized component will pass through the polarizing beam splitter cube into another polarizing beam splitter cube where it will recombine with the S-polarized beam that has either been delayed by mirrors or by a coil of optical fiber with a third polarizing beam splitter 
(since the fiber won't preserve polarization). By splitting the optical beams into two polarizations to excite the material or thin film of interest at different points in the time domain it is possible to cut the time of typical TIRPAS and QTPAS testing in half to test both polarization states. Once the two refractive index values are known we will have an idea of the birefringence of the material that adds an additional layer of characterization to most materials.

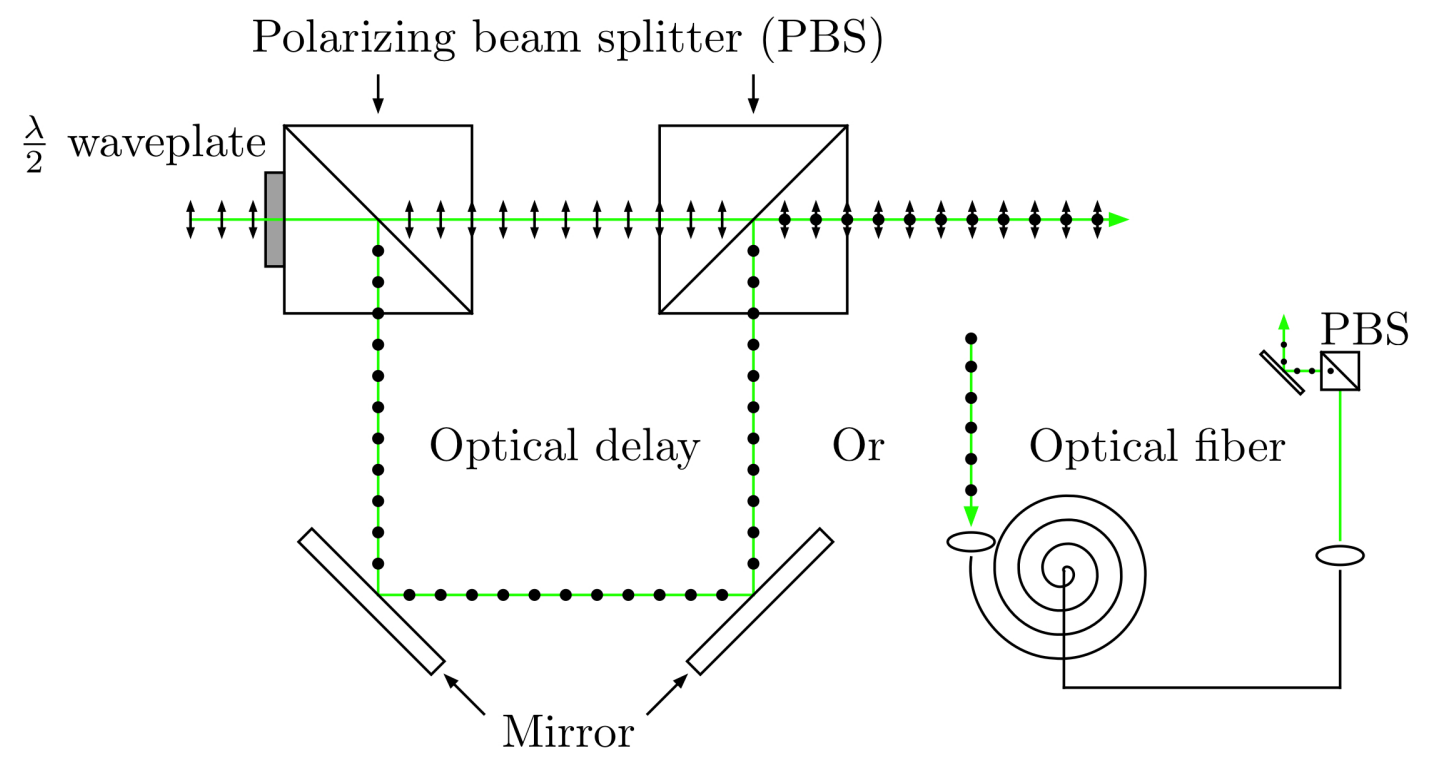

Figure 6.1: This idea uses dual polarizations of light to estimate birefringence.

\subsection{Optically Resonant TIRPAS}

Although TIRPAS has been shown to be able to detect as low as $1.1 \mathrm{~cm}^{-1}$ there are some materials that may have a very limited quantity of optical absorption such as dilute absorbers dissolved into water or biological fluids that are diagnostically important. In order to create an excellent biosensing platform the sensitivity of 
TIRPAS must be increased with the primary method of doing this being to create a resonant cavity to perform TIRPAS.

\subsubsection{0\% Absorption}

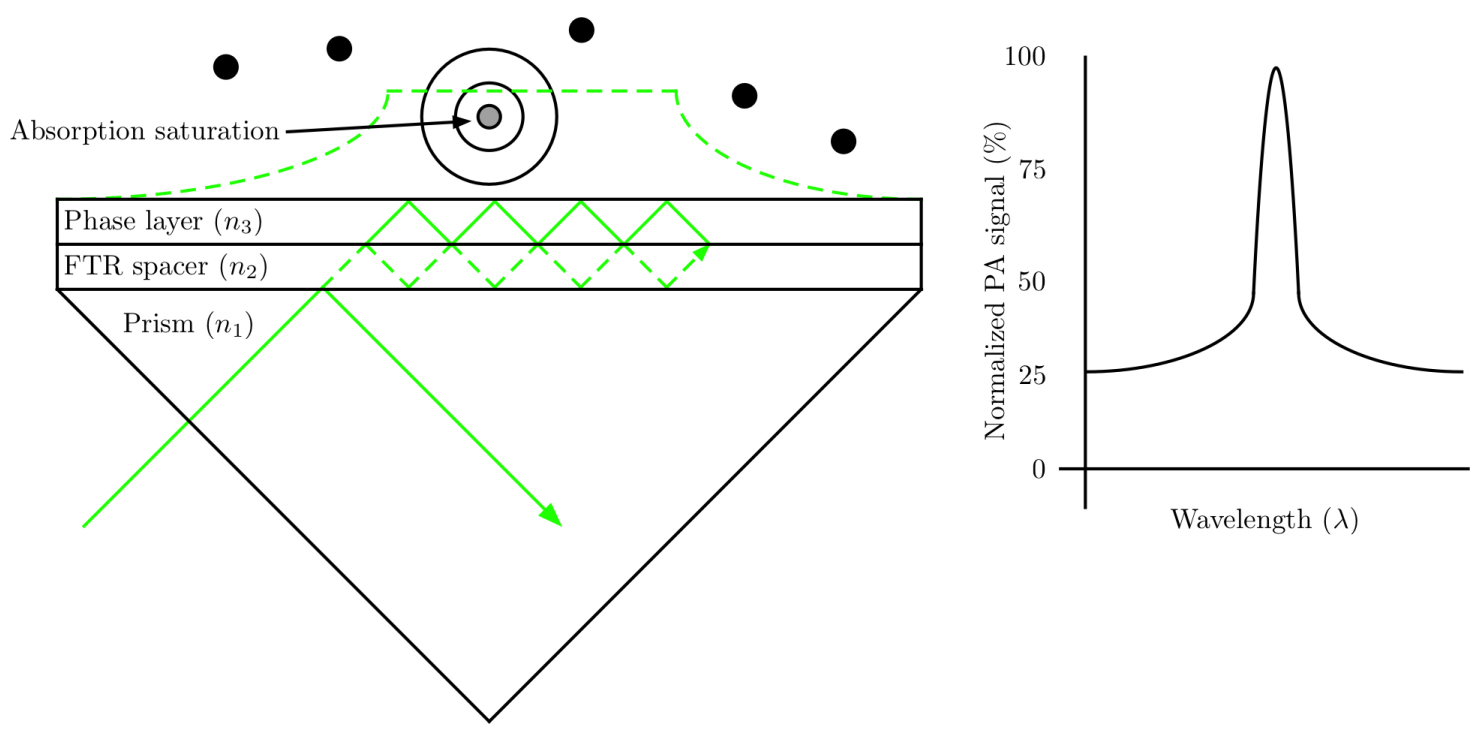

Figure 6.2: This figure shows a thin film resonator which can achieve a theoretical $100 \%$ optical absorption.

The major advantage of depositing a thin film resonator on a prism is that in principle it is possible to achieve $100 \%$ absorption of light into an analyte of interest. This is extremely important since it allows for the highest possible signal to noise ratio for a material. In addition, it is possible to optically saturate a material in absorption, which can be useful for optically pumping thin films for lasing studies. The important idea behind a 100\% absorption of the incident light is that this process would make TIRPAS as sensitive as it can physically be regardless of the absorbing material of interest. Since TIRPAS already constrains the optical penetration depth 
via the evanescent field $\sim 10$ times tighter than that obtainable in normal incidence photoacoustic excitation, it is in principle possible to increase the sensitivity of the photoacoustic effect by approximately 100 fold compared to standard photoacoustic excitation simply by adding a few thin films to a prism or substrate surface.

\subsection{Porosity Measurements}

Beyond simple characterization of the refractive index and thickness it is important to characterize other properties of the films that may be of interest such as the volume of the pores to calculate the porosity. This is particularly important for the development of mesoporous and microporous thin films with low dielectric values since porosity is a key factor in reducing the dielectric value for standard materials. Using the equation obtained from [139] we can find the porosity, $\rho$ given by

$$
\frac{n_{c}^{2}-1}{n_{c}^{2}+2}=(1-\rho) \frac{n_{b}^{2}-1}{n_{b}^{2}+2},
$$

where $n_{b}$ is the refractive index of the non-porous material, $n_{c}$ is the refractive index of the porous material, and $\rho$ is the porosity. The physical experiments necessary to do this calculation would be simple since the refractive index of most non-porous materials are already known in the visible spectrum and are available online. Once we calculate the porosity of the thin films we can do a variety of things such as monitor the absorption of materials into the films by measuring the refractive index over periods of absorption. 


\subsection{Optical Detection}

TIRPAS, although currently limited to liquid and solid materials due to the large difference in refractive index between gases and ultrasonic transducers, can in principle excite gases with the evanescent field the same way that the excitation occurs in liquids or solid samples. Over the years, several different optical methods [133-135] of photoacoustic detection have been created to overcome acoustic impedance limitations [140] between typical materials like PZT transducers and gases. However, since TIRPAS uses a prism/sample interface for excitation, it makes sense to use an optical method that includes a prism as well due to convenience.

Paultauf et al. [102] created a method using the change in refractive index induced due to acoustic wave propagation to optically detection photoacoustic excitation. The ideal quality of the optical detector is that the acoustic wave generated at the interface between the prism and sample is picked up by the photodetector sensing a change in light intensity rather than an acoustic wave activating a piezoelectric element that makes the differences in acoustic impedance meaningless. Therefore, optical detection of gases using TIRPAS should be feasible with this technique.

\subsection{Absorbing Thin Films}

Optical tunneling can happen in both transparent films as well as highly absorbing films. While I have been able to create a fully functional Labview program to characterize the optical tunneling effects through thin films, one of the assumptions in QTPAS is that the films do not absorb any of the evanescent field created in the film itself. In highly absorbing thin film materials such as solar cells there is significant 
absorption within the thin film and therefore more general equations need to be used to account for the absorption within the films as well as the optical tunneling effects. 


\section{Chapter 7}

\section{Summary and Concluding Remarks}

I have presented several new photoacoustic technologies based upon the interaction of the pulsed evanescent field with everything from highly absorbing dyes to biologically relevant analytes for heart attacks and malarial infection to mesoporous nanofilms. The importance of developing new characterization technologies, such as TIRPAS/(PAS/TIRPAS) refractometry/QTPAS, to probe the material properties of thin films, and biomarkers has been discussed at length in Chapter 1. In Chapter 2, using Maxwell's equations, the derivation of the existence of the evanescent field was presented in tandem with equations describing the photoacoustic effect and the qualitative effects of combining both into TIRPAS. Chapter 3 presents several experiments to show the potential to use TIRPAS as a biosensing technique for the detection of hemozoin, an optically pigmented waste product of a malarial infection in humans. A simple photoacoustic critical angle analysis technique, (PAS/TIRPAS) refractometry, was developed and tested on highly absorbing dyes and myoglobin in Chapter 4. Chapter 5 detailed the derivation of, current experiments, assumptions, 
and modeling of a technique that could determine the refractive index and thickness of nanofilms by combining the photoacoustic effect with optical tunneling (QTPAS). Finally, future directions for TIRPAS, (PAS/TIRPAS) refractometry, and QTPAS are presented with substantial background for reproduction in Chapter 6 .

\subsection{Modern TIRPAS and Biosensing}

Experiments showing the first example of the TIRPAS technique with nanosecond lasers were performed with the explicit purpose of detecting and characterizing the response of Chlorazol black dye and $\beta$-hematin for the purpose of using the TIRPAS technique as a biosensor for malarial infection. A proportion for the changes in photoacoustic signal was given an update for dual polarization excitation for the signal. It was shown through experiments comparing the oblique photoacoustic excitation regime that $\beta$-hematin and Chlorazol black give substantially different optical responses due to optical scattering.

These responses were compared with the responses of Chlorazol black dye and $\beta$-hematin for TIRPAS excitation and it was illustrated using representative examples that the acoustic response was very similar between the two materials suggesting that optical scattering could be somewhat dismissed due to the lower inherent optical penetration depth in TIRPAS. It was important to note that all TIRPAS acoustic waves imparted a plane wave like response regardless of the optical absorption/scattering tested. This was explained by the much larger aspect ratio of the photoacoustic excitation than typical techniques since the optical penetration depth is on the order

of $500 \mathrm{~nm}$ while the irradiation spot size is nearly always 1000 fold larger for experi- 
ments. It is currently unknown if it is even possible to obtain a spherical wave from TIRPAS experiments.

\subsection{Photoacoustic Refractometry (PAS/TIRPAS) and Biosensing}

Experiments were conducted to show the predictive ability of a photoacoustic refractometer (PAS/TIRPAS refractometry) to predict the refractive index of dye solutions and myoglobin for biosensing applications. The refractometer was compared to a standard handheld refractometer to correspond to the results. A Labview program was created to run the refractometry testing and reduce overall system noise through the use of a computational filter and waveform averaging. The PAS/TIRPAS refractometry technique allowed the photoacoustic effect to estimate the real component of refractive index of a material despite the photoacoustic effect being used traditionally to determine the complex component of the refractive index by finding $\mu_{a}$ This represented a step forward in technological development for photoacoustic technologies as it will allow the technique to find more optical properties beyond absorption and performing imaging. Additional modifications to the setup used the direct beam from a laser so that minimum distortion from optical divergence would be observed, eliminating the need for the spatial filter in Chapter 3. Finally, a process for finding initial estimates for the critical angle from the angular spectrum using a Stineman smoothing function and a numerical derivative from Kaleidagraph 4.1.0 allowed for the estimation of the refractive index of dyes and myoglobin. 


\subsection{QTPAS and Thin Films}

A technology (QTPAS) showcasing the potential to estimate the refractive index and thickness of a thin film was created and data was compared to a representative sample simulation for continued research in ongoing work. The potential to use this photoacoustic technique to evaluate the materials at wavelengths where the material is optically transparent and traditional photoacoustic evaluation would be unsuitable explains the true nature of the technique in revolutionizing the way the scientific community uses the photoacoustic effect. To date, there is no other photoacoustic technique that could scan a material without inherent optical absorption and potentially estimate material properties. Optical tunneling coupled with the photoacoustic effect seems to suggest the potential to expand the photoacoustic technique to optically transparent materials. Ongoing experimental evidence shows additional research is needed to fully realize the potential of the technique due to currently unknown complex effects involving the laser pulse energy and measured refractive index of the film of interest.

\subsection{Conclusion}

Traditional photoacoustic spectroscopy has illuminated many areas of biomedical research by reducing the importance of optical scattering for tissue and burn depth imaging. Additionally, the photoacoustic effect has changed the landscape of labelfree detection of circulating melanoma cells for fast and inexpensive diagnosis, characterization, and capture to usher in a new age of personalized medicine. However, biomedical imaging and flow cytometry are not the only avenues of research for the 
photoacoustic effect. With the combination of the pulsed evanescent field and the photoacoustic effect, I have revealed that there are still substantial unexplored avenues for the photoacoustic effect for the detection and characterization of materials at substrate/film or substrate/sample interfaces. The ability to detect the surface properties of materials while ignoring the bulk properties allows for the photoacoustic effect to evaluate material properties that it has never had access to before. The photoacoustic effect has shown the exceedingly helpful ability to evaluate materials at all scales except substantially at the nanoscale due to laser pulse duration limitations. With the invention of TIRPAS, (PAS/TIRPAS) refractometry, and QTPAS, the nano world can finally be substantially evaluated with photoacoustics without the need for expensive light sources or high frequency ultrasonic equipment. The potential to scan a transparent material with the photoacoustic effect without exogenous chromophores or destructive dielectric breakdown techniques will help bring into focus the nano world of photoacoustics by allowing the effect to work at scales from imaging an entire human body to evaluating the thickness of a nano film. 


\section{Appendix A}

\section{Various Important Issues}

There are several important experimental procedures or mathematical descriptions that are given here for completeness.

\section{A.1 Electrical Impedance Matching}

In all electrical systems mentioned in this dissertation it is important to have appropriate impedance matching for the systems and cabling. Electrical impedance refers to the real value and complex value of the resistance in wiring or devices as given by

$$
\Gamma_{12}=\frac{Z_{2}-Z_{1}}{Z_{2}+Z_{1}},
$$

where $\Gamma_{12}$ is the voltage reflection from impedance 1 to impedance 2 , and where $Z_{2}$ and $Z_{1}$ are the characteristic impedances. A typical reflection that occurs in laboratory equipment is between $50 \Omega$ and $75 \Omega \mathrm{BNC}$ cables. Even with a small difference 
between the two $20 \%$ reflection will result which can affect measurements from all sorts of devices. With regard to TIRPAS and QTPAS, the impedance matching is particularly important since the signals generated by the evanescent field and those signals generated by oblique absorption may have substantially different frequency responses which can distort the measured voltage values and give biased results. All

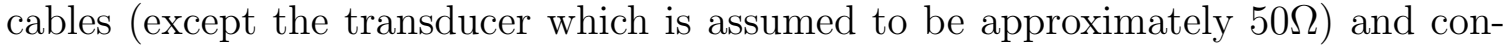
necting devices are $50 \Omega$ unless otherwise specified for the experiments.

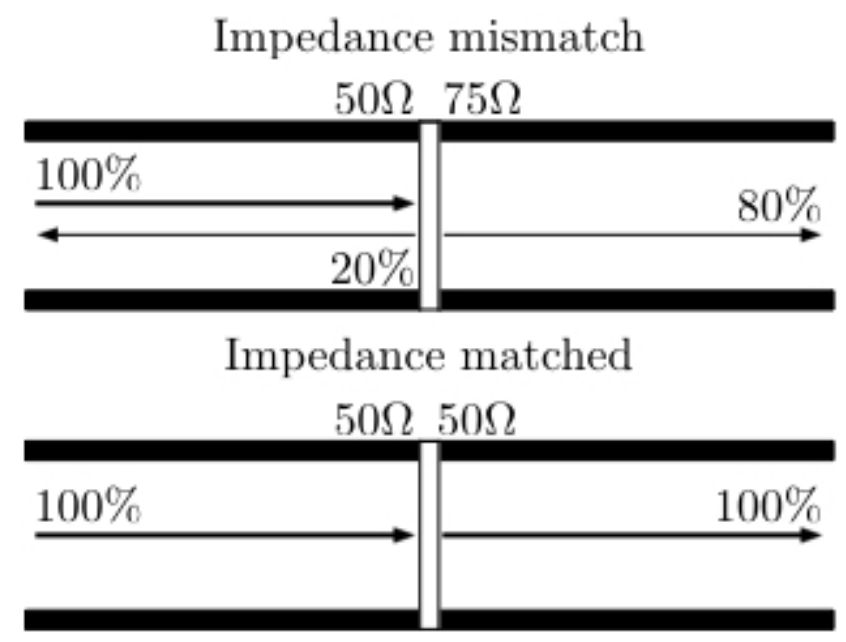

Figure A.1: Electrical impedance matching for signal integrity is very important to achieve an acceptable signal to noise ratio.

\section{A.2 Acoustic Impedance Matching}

Acoustic impedance is mathematically analogous to electrical impedance and to optical refractive index. In order to have a high signal to noise ratio for measurements it is important to match the impedance of the medium being tested and the transducer/any materials in-between given by 


$$
\Gamma_{12}=\frac{Z_{2}-Z_{1}}{Z_{2}+Z_{1}}
$$

Typical materials such as water have an acoustic impedance of 1.5 Mrayls whereas stainless steel is 45.7 Mrayls. The reflection from a water/stainless steel interface is $\sim 93 \%$. Since there is such a large difference between the acoustic impedance of liquids and solids, photoacoustic experiments have to take into account this difference and use materials that approximately match each other to reduce acoustic reflections and thus increase signal to noise ratio.

\section{A.3 Stineman Smoothing Algorithm from Kalei- dagraph 4.1.0}

The smoothing function in Kaleidagraph's software is based upon a combination of the Stineman interpolation method [128] with a weighting function. The step by step method is given below:

1. Fit the Stineman interpolation function to the data.

2. Apply a weighting function to the data to smooth the curve.

The weighting function takes into account the surrounding $10 \%$ of the data points during smoothing which can be understood better by a plot of the distribution in Fig. A.2. This is only true if $10 \%$ or more of the data is remaining on either side. If less is remaining than $10 \%$ of the data the function uses all data that is left until at the final point on the edge of the dataset the value of the Stineman interpolation is used. An array is then created using the following code: 


$$
\begin{aligned}
& \text { coeff }[\text { wsize- } 1]=1.0 \\
& \text { coeff }[\mathrm{kk}]=\text { coeff }[\mathrm{kk}+1]^{*} \text { magic }
\end{aligned}
$$

where wsize is $10 \%$ of the number of data points, magic $=\frac{1}{10^{\text {abs (wsize })}}$ and $\mathrm{kk}$ goes from 0 to (wsize-2). From there the weighting function is applied to the data and the smooth curve is output to the program.

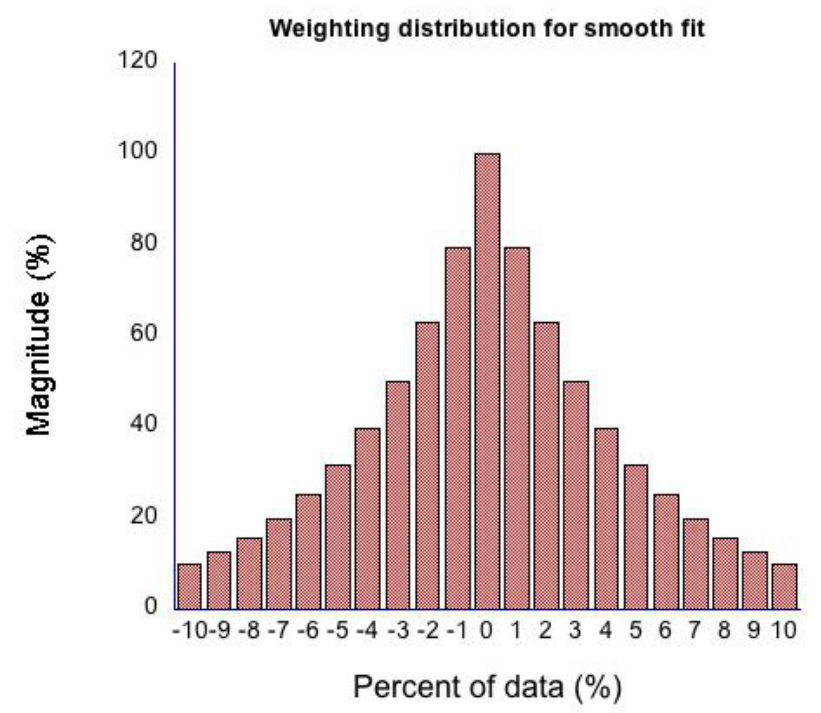

Figure A.2: This figure shows the weighting structure for the Stineman smooth function.

\section{A.4 Electromagnetic Noise Considerations}

Due to the very sensitive nature of TIRPAS and QTPAS, our setups had to be designed in such a way to reduce electromagnetic noise in as many ways as possible. For completeness, a more comprehensive explanation of our methods is given here. 


\section{A.4.1 Ground Loop Noise}

Both TIRPAS and QTPAS use National Instruments' Labview programming environment to scan materials. In order to completely automate the system so that no optical movement is needed, a stepper motor driver, the MID 7604, along with a Sherline rotational stage is needed to measure optical tunneling and evanescent absorption. Traditional photoacoustic experiments simply rely upon a single-ended amplifier for signal acquisition. However, due to the fact that the high current stepper motors are directly connected to the same circuitry as the transducer, amplifier, computer, and PXI system, any electronic noise generated by the stepper motors will result in measured output noise in the signal due to ground loops.

\section{A.4.2 What is a Ground Loop?}

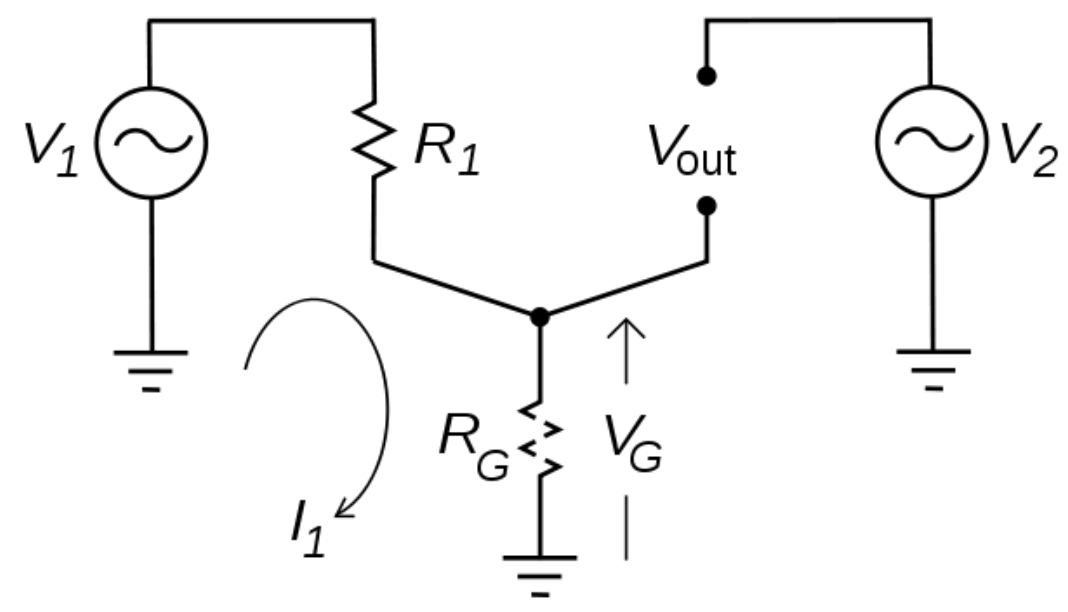

Figure A.3: This figure shows the circuit diagram of a ground loop.

A ground loop, as shown in Figure A.3, occurs when two devices are grounded to two 
potential grounds that are assumed to be at the same potential, but in fact are not. When the two grounds are at different potentials it creates the situation where an unwanted current can flow between them causing signal contamination in the form of common mode noise, it can cause component damage, and in some extraordinary cases even human harm in the form of electrocution.

In terms of how ground loops affect overall measurement in the TIRPAS system, there is a noisy signal trace versus angle which is particularly emphasized at higher angles where the signal to noise ratio is lower. The noise presents itself as semi-random spikes of peak of peak voltage as shown in Figure A.4.

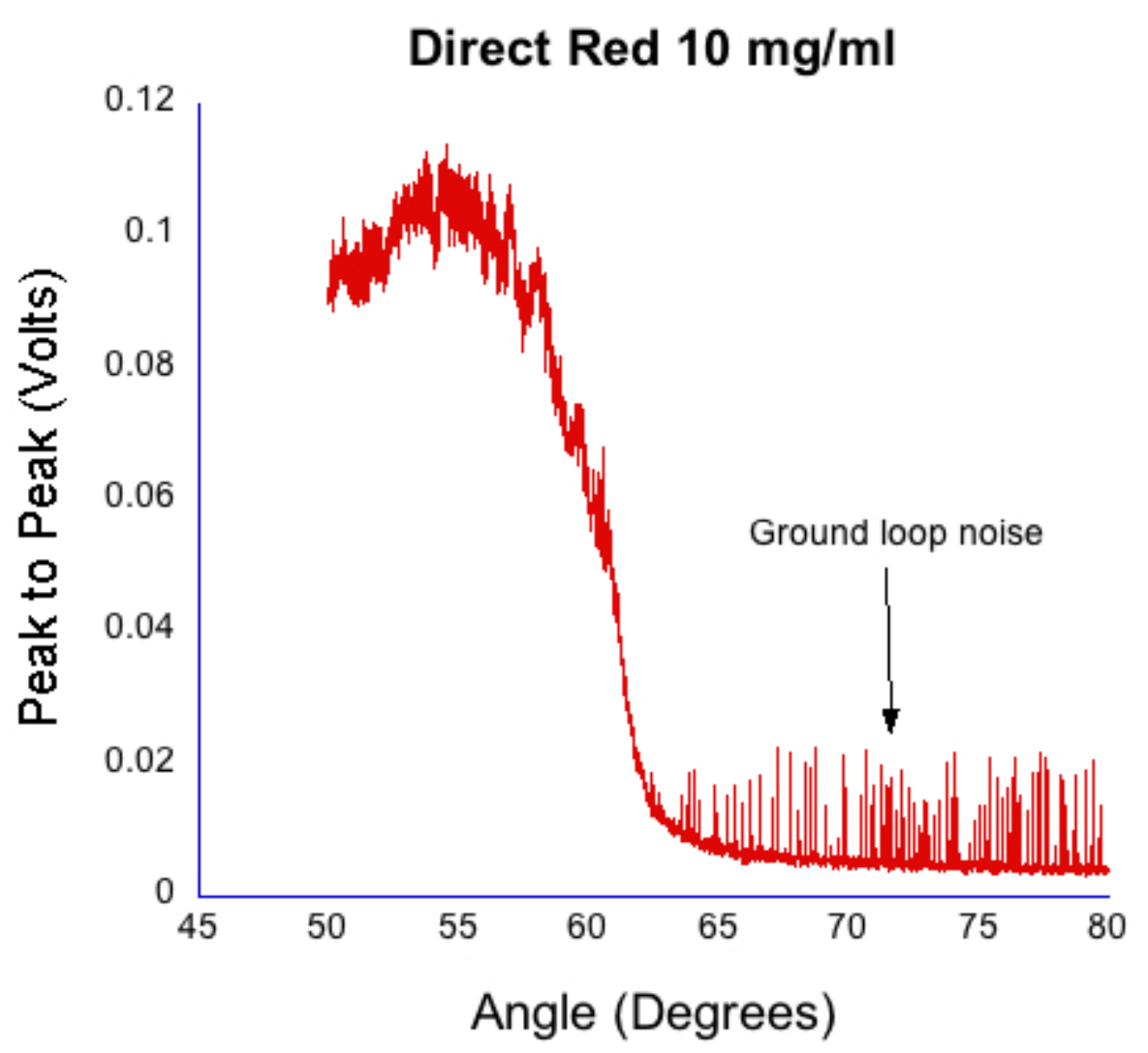

Figure A.4: TIRPAS angular spectra sometimes receive noise via ground loops. 


\section{A.4.3 How does a Differential Amplifier Help?}

A differential amplifier, as explained in detail in [141], helps alleviate the issue of ground loop noise by only amplifying the difference between two inputs + and -. Since both inputs share the ground loop noise or common mode noise caused by the unavoidable interconnections in the system the differential amplifier simply cancels them out and the resulting signal is free from that form of noise.

\section{A.4.4 Inductive Noise}

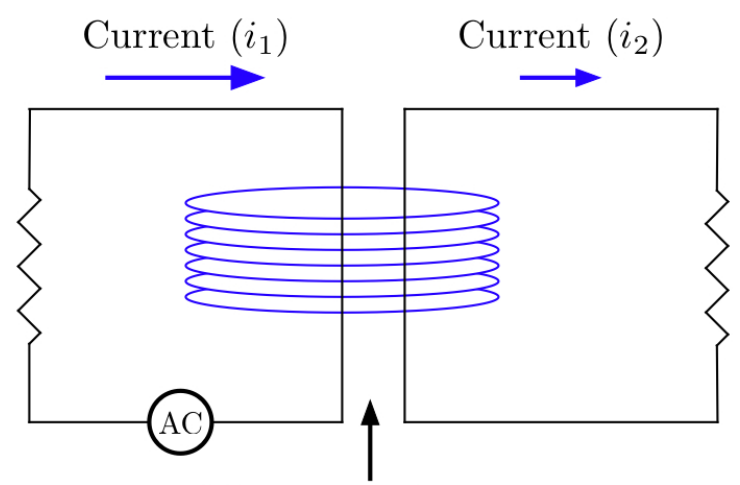

Magnetic induction

Figure A.5: Noise can occur via magnetic induction as well as ground loops.

Inductive noise is noise generated by the interaction of two conductors with overlapping magnetic fields as shown in Figure A.5. This process is the basis of electrical transformers, however, this presents the issue of increased signal noise for typically configured equipment that is within close proximity to other noisy equipment. For TIRPAS and QTPAS, the motor that allows for the automation of the system is physically close to the sensing element (transducer) and can inductively couple motor noise into the system. Unfortunately, the electronic noise generated through this pro- 
cess has a similar frequency spectrum to the electrical representation of the acoustic signals and therefore, computational filtering is not possible. In Figure A.6 we see the effect of inductive noise on a typical spectrum. At the lower angles of incidence, the noise seems to be large but as the angles increase the noise seems to subside. This fluctuation of the noise versus angle of incidence has to do with the fact that at lower angles of incidence, the transducer and wiring is nearly parallel with the motor and wiring whereas at higher angles of incidence the transducer and the motor are nearly perpendicular to one another. This difference in alignment is important because the most efficient induction of charge from one wire into another occurs whenever the two wires are parallel to each other. Almost no inductive coupling occurs between two wires when they are perpendicular to each other due to the increased distance between the wires everywhere but at the intersection of the perpendicular.

\section{A.4.5 Quantization Noise}

The final major source of noise in the system comes from the nature of converting an analog signal to a digital signal in a real world application. Quantization noise is the effect where an analog signal (which can take on an infinite number of values) is digitized into discrete levels that contain fewer values than that in the set of numbers that correspond to an analog signal. This compression causes information loss in A/D converters by rounding off a value such as 3.445631 to 3.4 by the nature of how many digitization levels can be obtained by a digitizer. In the case of the PXI-5152, only 8-bit is available, thus only 256 levels can represent any one signal. With the smallest voltage range enabled, this means that the smallest value of voltage that can reliably be measured is $0.390625 \mathrm{mV}$ out of a possible $100 \mathrm{mV}$. What this means 
is that a small measured signal may only be recreated with a few points which may introduce distortion in the overall shape of the waveform due to only discrete voltage levels being possible values as shown in Figure A.7.

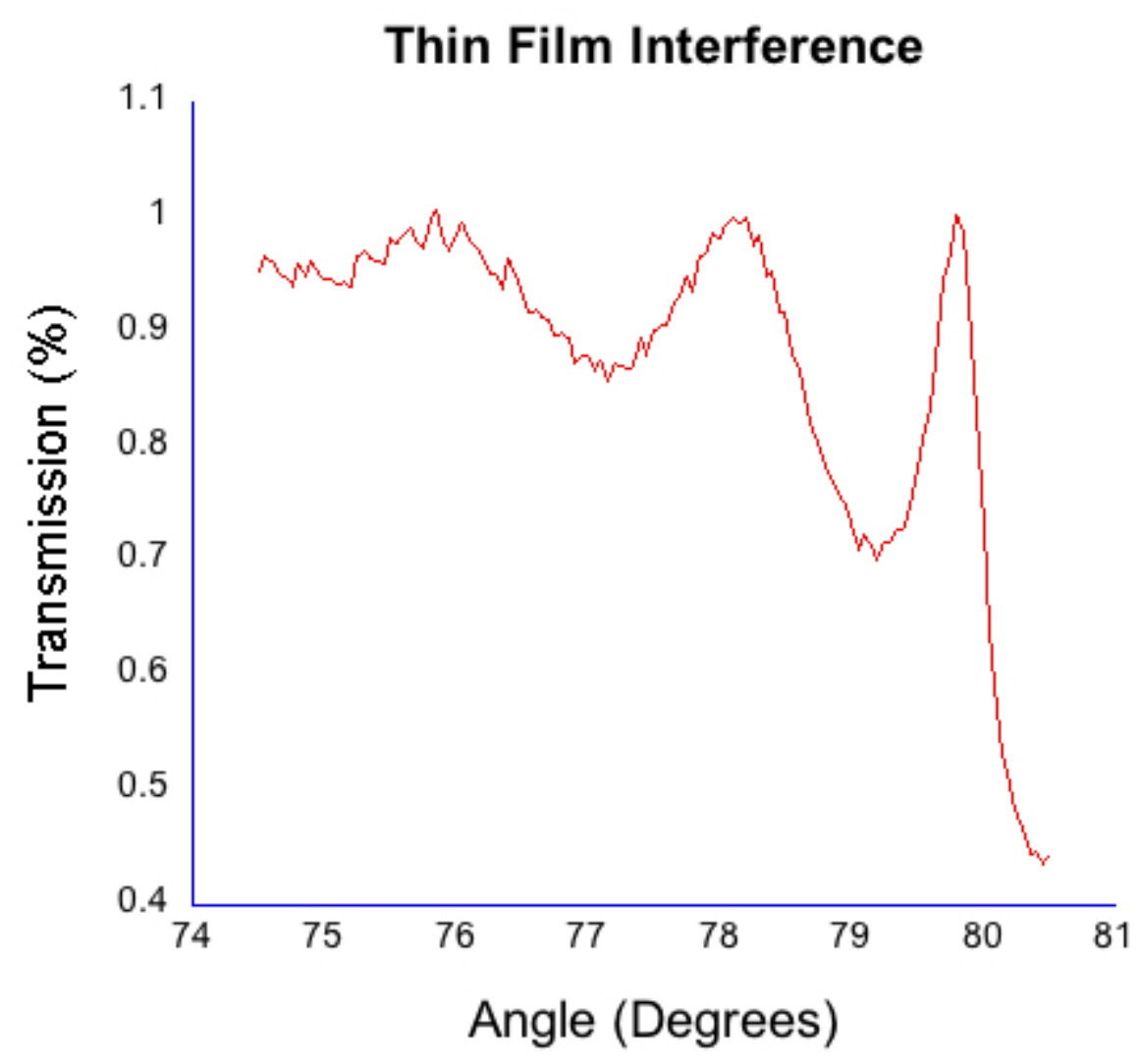

Figure A.6: This figure shows an angular scan with inductive noise.

\section{A.4.6 Dithering}

Many researchers have used a technique called "dithering" to reduce or eliminate quantization noise for signals that can be averaged. Dithering intentionally introduces a random source of noise to the analog signal in order to make the value fluctuate 


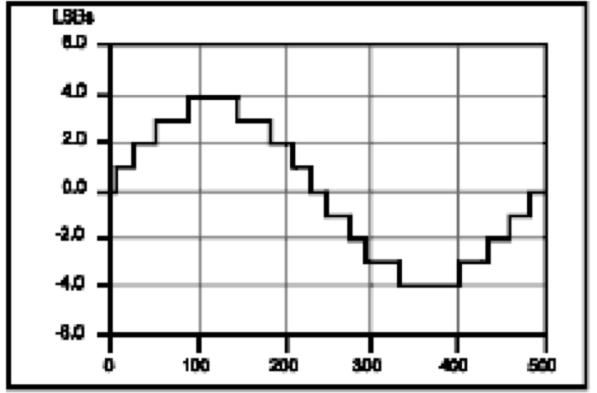

a. Dither disabled; no averaging

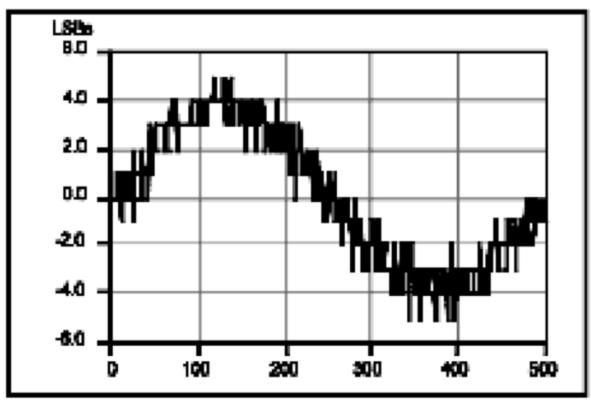

c. Dither enabled; no averaging

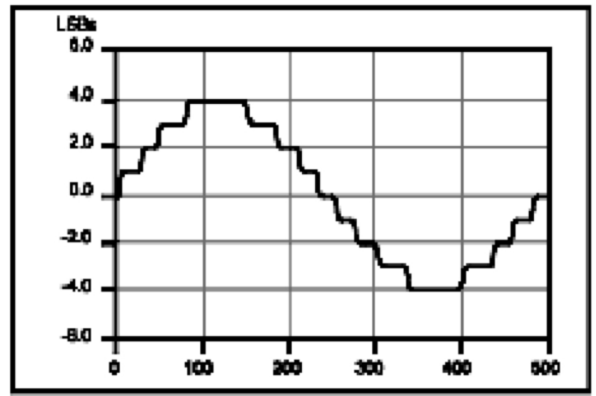

b. Dither disabled; average of 50 acquisitions

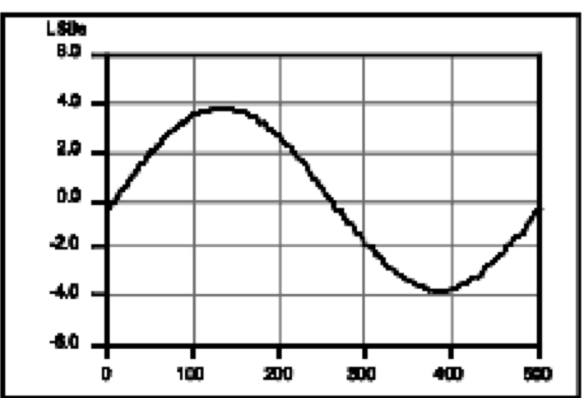

d. Dither enabled; average of 50 acquisitions

Figure A.7: Dithering can increase vertical digitizer resolution. (Reproduced with permission from National Instruments at http://www.ni.com/white-paper/3016/ en/)

between two adjacent quantization levels. This process is demonstrated in Figure A.7 where the sinusoidal waveform without dithering is shown averaged and without averaging to show substantial artifacts introduced as steps via quantization. However, when random noise is added to the signal (dithering) after averaging the sinusoidal signal is clearly more accurately depicted. This is due to the fact that the noise is random such that after averaging the step quantization effect is cancelled out and a waveform closer to the analog signal is reproduced as shown in Figure A.7. 


\section{A.5 QTPAS Flow Chart}

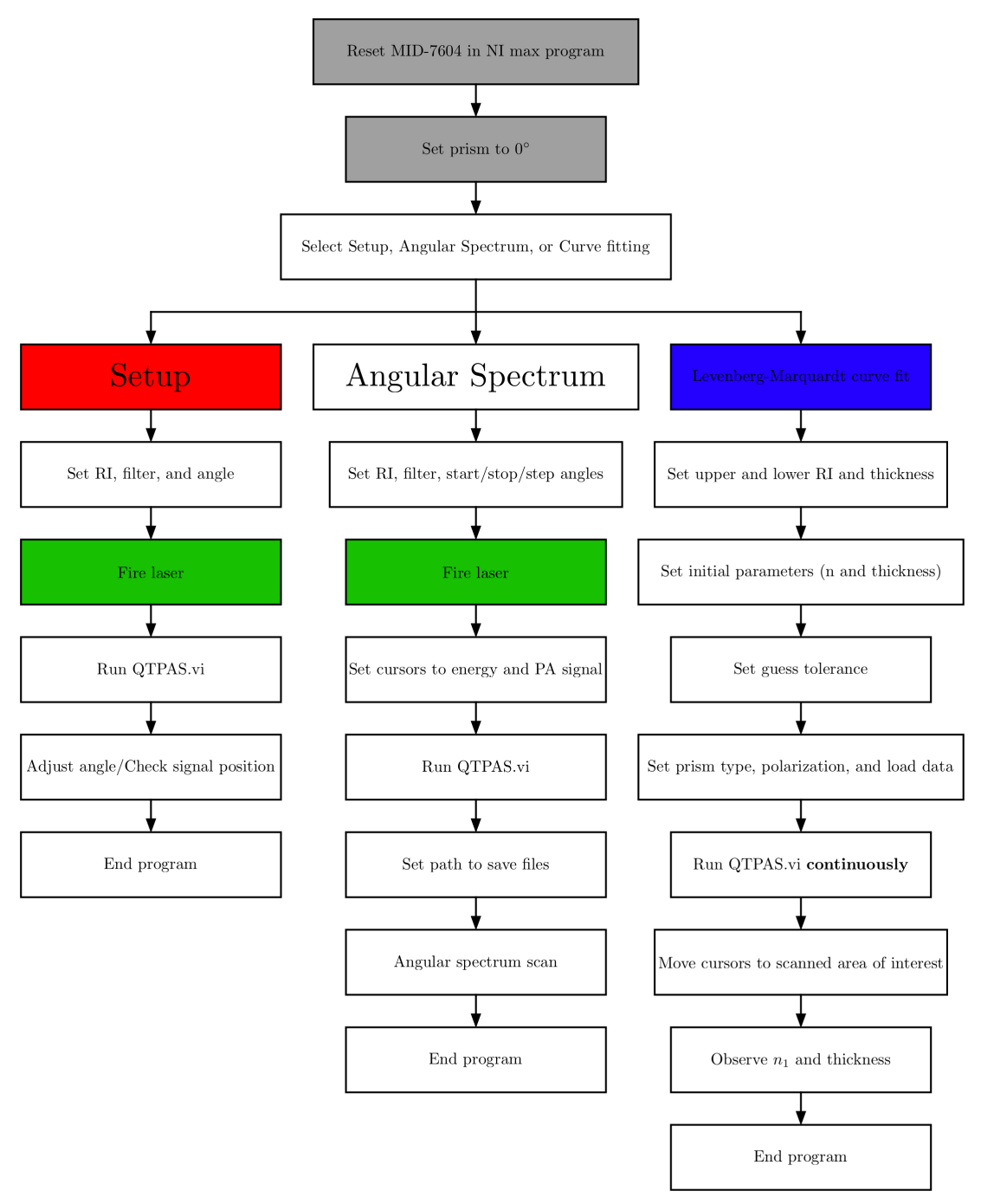

Figure A.8: This flow chart explains the QTPAS Labview/experimental program from the beginning to the end of the experiment.

Following is the flow chart for the Quantum Tunneling Photoacoustic Spectroscopy virtual instrument. Three separate modes, Setup, Angular Spectrum, and Curve fitting are available for operating the system. 


\section{A.6 Signal Simulation: ModelingQTPAS.m}

Following is the code used to generate the Gaussian simulated signal for QTPAS.

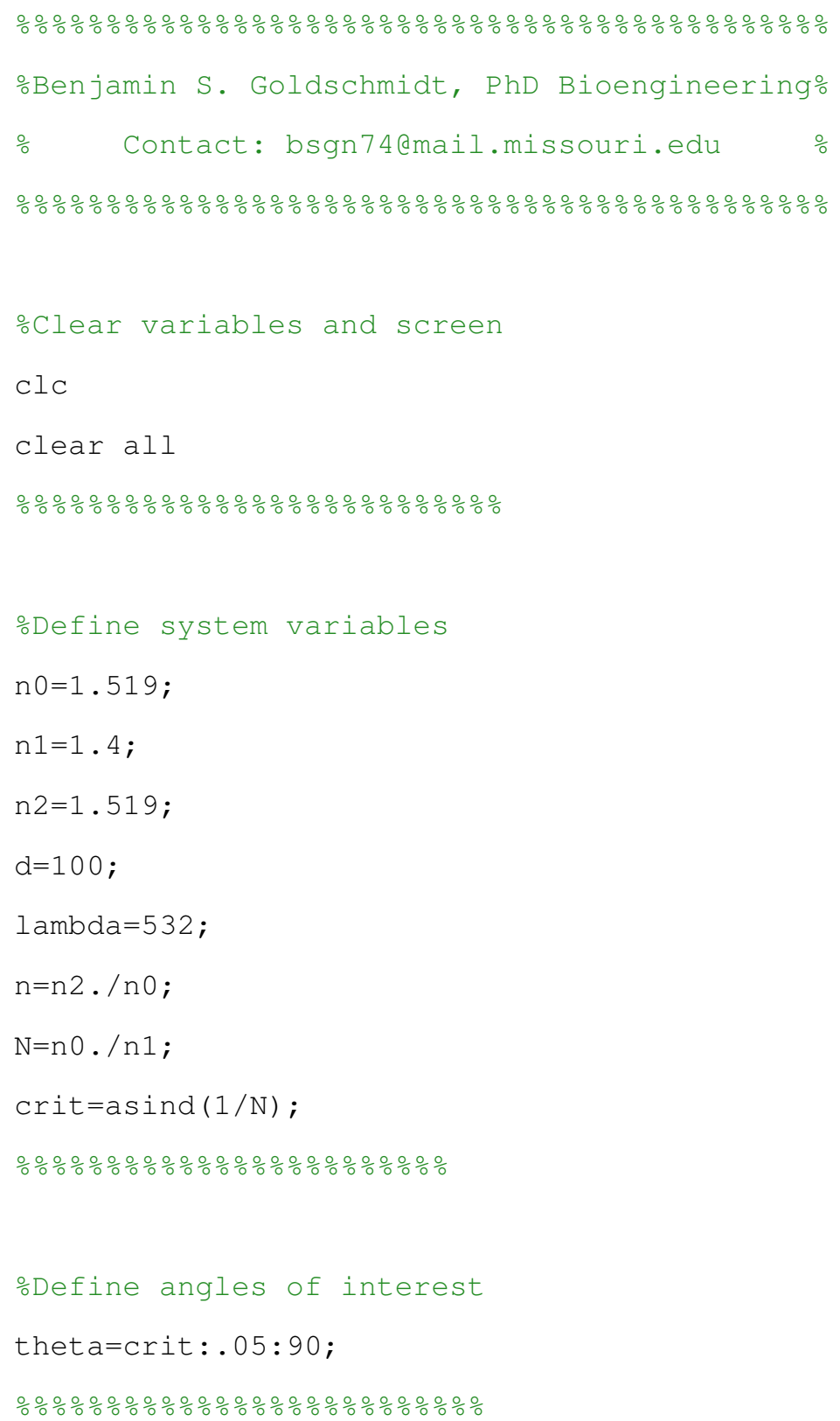




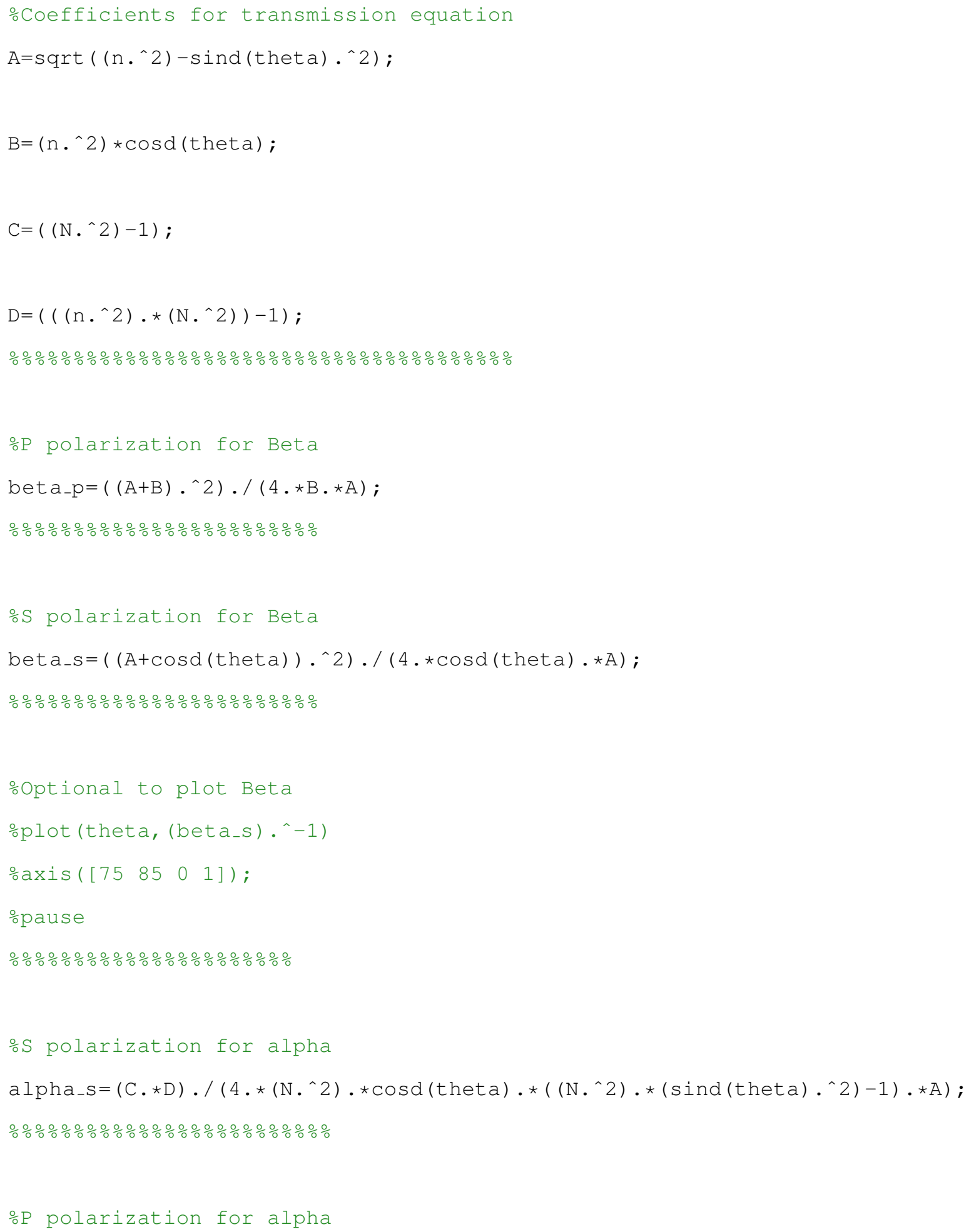




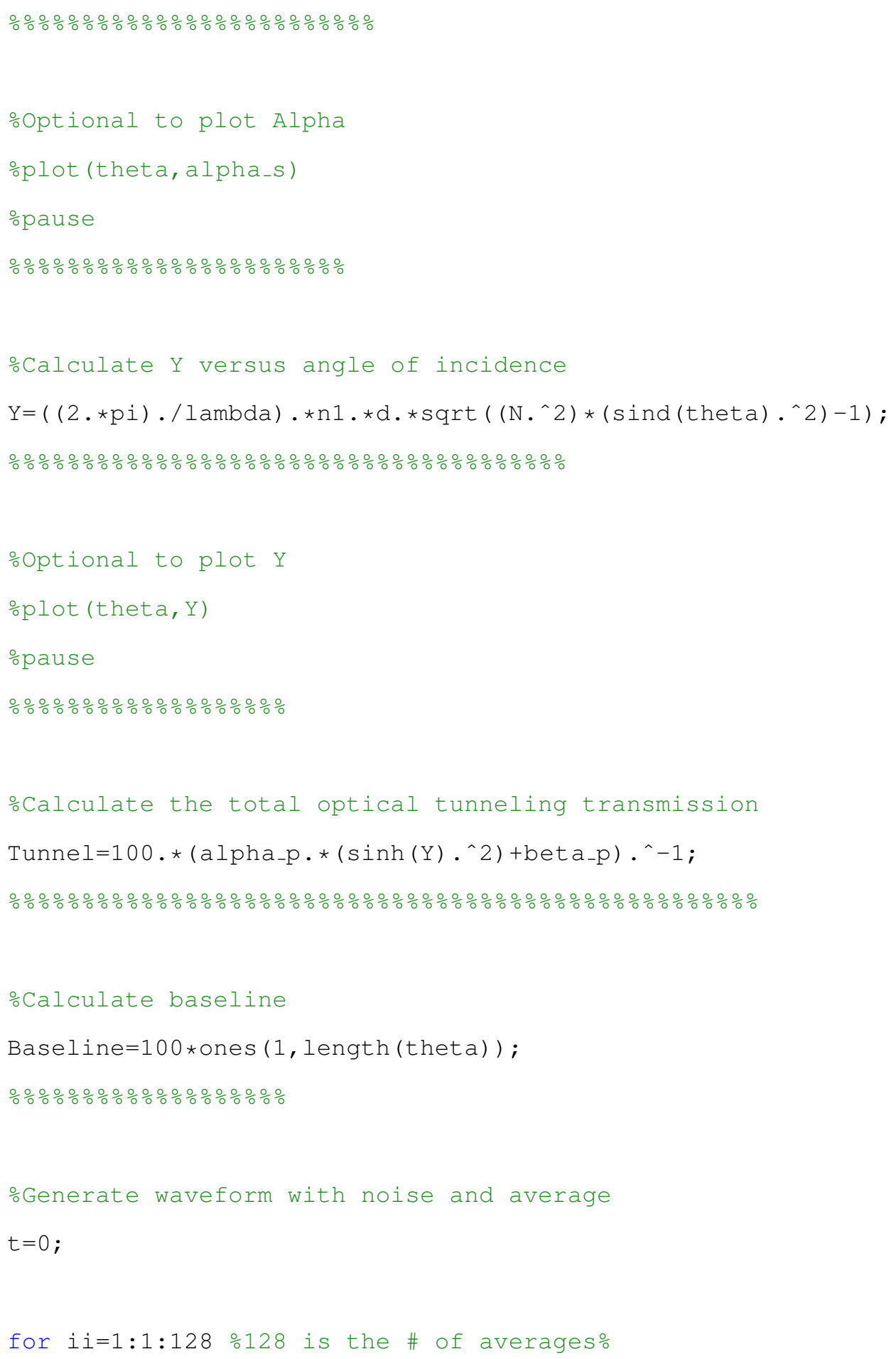




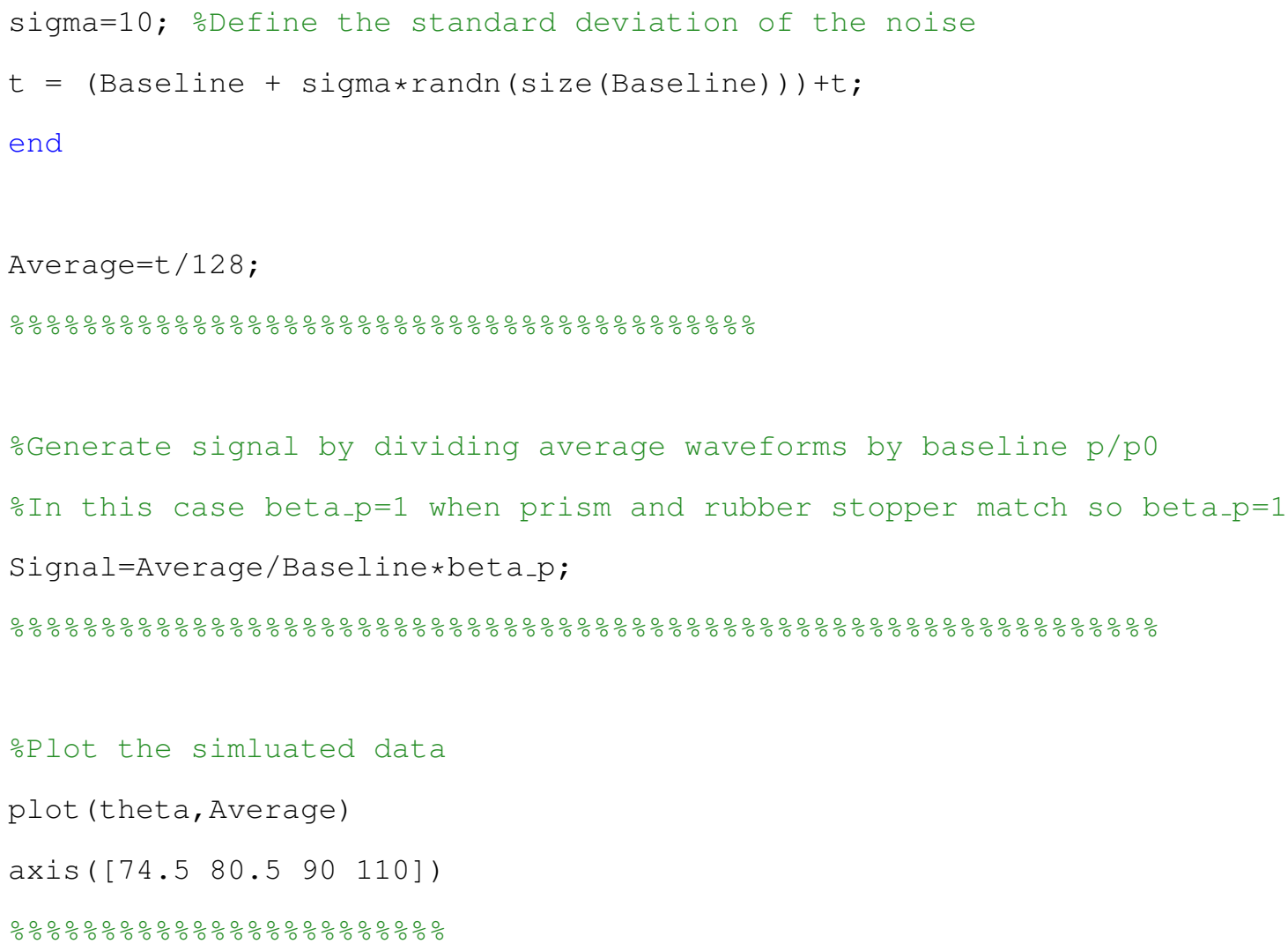




\section{Bibliography}

[1] T.S. Glazneva, E.V. Rebrov, J.C. Schouten, E.A. Paukshtis, and Z.R. Ismagilov. Synthesis and characterization of mesoporous silica thin films as a catalyst support on a titanium substrate. Thin Solid Films, 515(16):6391-6394, 2007.

[2] Wenjing Liu, Zhening Zhu, Ke Deng, Zhengtao Li, Yunlong Zhou, Huibin Qiu, Yan Gao, Shunai Che, and Zhiyong Tang. Gold nanorod@chiral mesoporous silica core-shell nanoparticles with unique optical properties. Journal of the American Chemical Society, 135(26):9659-9664, 2013.

[3] Taewook Kang, Seogil Oh, Surin Hong, Jungwoo Moon, and Jongheop Yi. Mesoporous silica thin films as a spatially extended probe of interfacial electric fields for amplified signal transduction in surface plasmon resonance spectroscopy. Chem. Commun., pages 2998-3000, 2006.

[4] Jinghua Sun, Baohu Wu, Hongbao Jia, Dong Wu, and Yao Xu. Fluoroalkylgrafted mesoporous silica antireflective films with enhanced stability in vacuum. Opt. Lett., 37(19):4095-4097, Oct 2012.

[5] T. Yamada, H.-S. Zhou, H. Uchida, M. Tomita, Y. Ueno, T. Ichino, I. Honma, K. Asai, and T. Katsube. Surface photovoltage no gas sensor with properties de- 
pendent on the structure of the self-ordered mesoporous silicate film. Advanced Materials, 14(11):812-815, 2002.

[6] Xinglu Huang, Fan Zhang, Seulki Lee, Magdalena Swierczewska, Dale O. Kiesewetter, Lixin Lang, Guofeng Zhang, Lei Zhu, Haokao Gao, Hak Soo Choi, Gang Niu, and Xiaoyuan Chen. Long-term multimodal imaging of tumor draining sentinel lymph nodes using mesoporous silica-based nanoprobes. Biomaterials, 33(17):4370 - 4378, 2012.

[7] K. Bhattacharyya, M. Njoroge, B.S. Goldschmidt, B. Gaffigan, K. Rood, and J.A. Viator. Detection, isolation, and capture of circulating breast cancer cells with photoacoustic flow cytometry. In Frontiers in Biological Detection: From Nanosensors to Systems V, volume 8570, 2013.

[8] T. Junno, S. Anand, K. Deppert, L. Montelius, and L. Samuelson. Contact mode atomic force microscopy imaging of nanometer-sized particles. Applied Physics Letters, 1995.

[9] David J. Marchand, Erik Hsiao, and Seong H. Kim. Non-contact afm imaging in water using electrically driven cantilever vibration. Langmuir, 29(22):67626769, 2013.

[10] P.K. Hansma, J.P. Cleveland, M. Radmacher, D.A. Walters, P.E. Hillner, M. Bezanilla, M. Fritz, D. Vie, H.G. Hansma, C.B. Prater, J. Massie, L. Fukunaga, J. Gurley, and V. Elings. Tapping mode atomic force microscopy in liquids. Applied Physics Letters, 1994. 
[11] R. Splinter and B.A. Hooper. An Introduction to Biomedical Optics (Optics and Optoelectronics). Taylor and Francis, Inc., Bristol, PA, USA, 2006.

[12] N. Chanda, R. Shukla, A. Zambre, S. Mekapothula, R.R. Kulkarni, K. Katti, K. Bhattacharyya, G.M. Fent, S.W. Casteel, E.J. Boote, J.A. Viator, A. Upendran, R. Kannan, and K.V. Katti. An effective strategy for the synthesis of biocompatible gold nanoparticles using cinnamon phytochemicals for phantom ct imaging and photoacoustic detection of cancerous cells. Pharmaceutical Research, 28(2):279-291, 2011.

[13] R.M. Weight, P.S. Dale, and J.A. Viator. Detection of circulating melanoma cells in human blood using photoacoustic flowmetry. Conference proceedings : ... Annual International Conference of the IEEE Engineering in Medicine and Biology Society. IEEE Engineering in Medicine and Biology Society. Conference, 2009:106-109, 2009.

[14] R.M. Weight, P.S. Dale, and J.A. Viator. Detection of circulating melanoma cells in human blood using photoacoustic flowmetry. In Annual International Conference of the IEEE Engineering in Medicine and Biology Society, 2009, pages 106-109, 2009.

[15] R.M. Weight and J.A. Viator. Detection of circulating tumor cells by photoacoustic flowmetry. Methods in Molecular Biology, 1102:655-663, 2014.

[16] G. Gutierrez-Juarez, S.K. Gupta, M. Al-Shaer, L. Polo-Parada, P.S. Dale, C. Papageorgio, and J.A. Viator. Detection of melanoma cells in vitro using an optical detector of photoacoustic waves. Lasers in Surgery and Medicine, 42(3):274-281, 2010. 
[17] E.M. Spradling and J.A. Viator. Detection of melanoma cells suspended in mononuclear cells and blood plasma using photoacoustic generation. In Photons Plus Ultrasound: Imaging and Sensing 2009, volume 7177, 2009.

[18] D.R. McCormack, K. Bhattacharyya, R. Kannan, K. Katti, and J.A. Viator. Enhanced detection of circulating melanoma cells using gold nanoparticles as photoacoustic contrasting agents. In Photons Plus Ultrasound: Imaging and Sensing 2010, volume 7564, 2010.

[19] J.A. Viator, S. Gupta, B.S. Goldschmidt, K. Bhattacharyya, R. Kannan, R. Shukla, P.S. Dale, E. Boote, and K. Katti. Gold nanoparticle mediated detection of prostate cancer cells using photoacoustic flowmetry with optical reflectance. Journal of Biomedical Nanotechnology, 6(2):187-191, 2010.

[20] K. Bhattacharyya, B.S. Goldschmidt, M. Hannink, S. Alexander, A. Jurkevic, and J.A. Viator. Gold nanoparticle-mediated detection of circulating cancer cells. Clinics in Laboratory Medicine, 32(1):89-101, 2012.

[21] T.S. Thomas, P.S. Dale, R.M. Weight, U. Atasoy, J. Magee, and J.A. Viator. Photoacoustic detection of breast cancer cells in human blood. In Photons Plus Ultrasound: Imaging and Sensing 2008: The Ninth Conference on Biomedical Thermoacoustics, Optoacoustics, and Acousto-optics, volume 6856, 2008.

[22] R.M. Weight, P.S. Dale, G. Gutierrez-Juarez, C.W. Caldwell, A.B. Lisle, and J.A. Viator. Photoacoustic detection of circulating melanoma cells, in vitro. In Photons Plus Ultrasound: Imaging and Sensing 200\%: The Eighth Conference on Biomedical Thermoacoustics, Optoacoustics, and Acousto-optics, volume 6437, 2007. 
[23] S.K. Gupta, K. Katti, and J.A. Viator. Photoacoustic detection of gold nanorods tagged prostate cancer cells in-vitro. In Photons Plus Ultrasound: Imaging and Sensing 2009, volume 7177, 2009.

[24] S. Gupta, K. Bhattacharya, J.R. Newton, T.P. Quinn, and J.A. Viator. Photoacoustic detection of induced melanoma in vitro using a mouse model. In Advanced Biomedical and Clinical Diagnostic Systems X, volume 8214, 2012.

[25] D. McCormack, M. Al-Shaer, B.S. Goldschmidt, P.S. Dale, C. Henry, C. Papageorgio, K. Bhattacharyya, and J.A. Viator. Photoacoustic detection of melanoma micrometastasis in sentinel lymph nodes. Journal of Biomechanical Engineering, 131(7), 2009.

[26] R.M. Weight, J.A. Viator, P.S. Dale, C.W. Caldwell, and A.E. Lisle. Photoacoustic detection of metastatic melanoma cells in the human circulatory system. Optics Letters, 31(20):2998-3000, 2006.

[27] S.H. Holan and J.A. Viator. Automated wavelet denoising of photoacoustic signals for circulating melanoma cell detection and burn image reconstruction. Physics in Medicine and Biology, 53(12):N227-N236, 2008.

[28] M.A. Juratli, M. Sarimollaoglu, D.A. Nedosekin, A.V. Melerzanov, V.P. Zharov, and E.I. Galanzha. Dynamic fluctuation of circulating tumor cells during cancer progression. Cancers, 6(1):128-142, 2014.

[29] J.A. Viator, P. Sutovsky, and R.M. Weight. Detection of dilute sperm samples using photoacoustic flowmetry. In Photons Plus Ultrasound: Imaging and 
Sensing 2008: The Ninth Conference on Biomedical Thermoacoustics, Optoacoustics, and Acousto-optics, volume 6856, 2008.

[30] Ekaterina I. Galanzha, Evgeny V. Shashkov, Valery V. Tuchin, and Vladimir P. Zharov. In vivo multispectral, multiparameter, photoacoustic lymph flow cytometry with natural cell focusing, label-free detection and multicolor nanoparticle probes. Cytometry Part A, 73A(10):884-894, 2008.

[31] J.A. Viator, J. Komadina, L.O. Svaasand, G. Aguilar, B. Choi, and J.S. Nelson. A comparative study of photoacoustic and reflectance methods for determination of epidermal melanin content. Journal of Investigative Dermatology, 122(6):1432-1439, 2004.

[32] S.H. Holan and J.A. Viator. Automated wavelet denoising of photoacoustic signals for burn depth image reconstruction. In Photons Plus Ultrasound: Imaging and Sensing 200\%: The Eighth Conference on Biomedical Thermoacoustics, Optoacoustics, and Acousto-optics, volume 6437, 2007.

[33] J.A. Viator, G. Au, G. Paltauf, S.L. Jacques, S.A. Prahl, H. Ren, Z. Chen, and J. Stuart Nelson. Clinical testing of a photoacoustic probe for port wine stain depth determination. Lasers in Surgery and Medicine, 30(2):141-148, 2002.

[34] J.A. Viator, G. Paltauf, S.L. Jacques, and S.A. Prahl. Design and testing of an endoscopic photoacoustic probe for determination of treatment depth after photodynamic therapy. In Biomedical Optoacoustics II, volume 4256, pages 16-27, 2001. 
[35] J.A. Viator, G. Au, B. Choi, and J.S. Nelson. Design limitations of a photoacoustic probe for port wine stain depth determination. In Biomedical Optoacoustics III, volume 4618, pages 7-15, 2002.

[36] J.A. Viator, S.L. Jacques, and G. Aguilar. Imaging of port wine stain lesions using a multi-sensor photoacoustic probe. In ASME 2004 International Mechanical Engineering Congress and Exposition, volume 375, pages 719-723, 2004 .

[37] J.A. Viator, B. Choi, M. Ambrose, J. Spanier, and J.S. Nelson. In vivo portwine stain depth determination with a photoacoustic probe. Applied Optics, 42(16):3215-3224, 2003.

[38] G. Paltauf, J.A. Viator, S.A. Prahl, and S.L. Jacques. Iterative reconstruction algorithm for optoacoustic imaging. Journal of the Acoustical Society of America, 112(4):1536-1544, 2002.

[39] G. Paltauf, J.A. Viator, S.A. Prahl, and S.L. Jacques. Iterative reconstruction method for three-dimensional optoacoustic imaging. In Biomedical Optoacoustics II, volume 4256, pages 138-146, 2001.

[40] R.J. Talbert and J.A. Viator. Limitation of photoacoustic burn depth measurement using monte carlo analysis. In Photons Plus Ultrasound: Imaging and Sensing 200\%: The Eighth Conference on Biomedical Thermoacoustics, Optoacoustics, and Acousto-optics, volume 6437, 2007.

[41] J.A. Viator and S.L. Jacques. Limitations of photoacoustic measurement of burn depth. In ASME 2005 Summer Heat Transfer Conference collocated with 
the ASME 2005 Pacific Rim Technical Conference and Exhibition on Integration and Packaging of MEMS, NEMS, and Electronic Systems, volume 1, pages 849$854,2005$.

[42] J.A. Viator, G. Paltauf, S.L. Jacques, and S.A. Prahl. Localization of spherical photoacoustic sources in acrylamide gels using time domain measurements. In Biomedical Optoacoustics, volume 3916, pages 89-99, 2000.

[43] J.A. Viator, G. Aguilar, S.L. Jacques, and J.S. Nelson. Optimization of cryogen spray cooling for port wine stain laser therapy using photoacoustic measurement of epidermal melanin. In ASME 2003 International Mechanical Engineering Congress and Exposition, volume 55, pages 13-14, 2003.

[44] Steven L. Jacques, John A. Viator, and G. Paltauf. Optoacoustic imaging of tissue blanching during photodynamic therapy of esophageal cancer. In Biomedical Optoacoustics, volume 3916, pages 322-330, 2000.

[45] J. Komadina, J.A. Viator, and J. Stuart Nelson. Photoacoustic diffraction studies of planar tissue phantoms. In Biomedical Optoacoustics IV, volume 4960, pages 58-63, 2003.

[46] S.L. Jacques, G. Palthauf, and J. A. Viator. Photoacoustic imaging in biological tissues with pulsed lasers. Annals of Biomedical Engineering, 28(SUPPL. 1):S39,2000 .

[47] J.A. Viator and S.A. Prahl. Photoacoustic imaging of gelatin phantoms using matched field processing. In Laser-Tissue Interaction X: Photochemical, Photothermal, and Photomechanical, volume 3601, pages 276-283, 1999. 
[48] J.A. Viator, S.H. Holan, E. Spradling, and R.J. Talbert. Determination of optical absorption coefficient of thermally coagulated blood using photoacoustic analysis. In ASME 2008 Summer Bioengineering Conference, pages 569-570, 2009 .

[49] J.A. Viator, S.L. Jacques, and S.A. Prahl. Generating subsurface acoustic waves in indocyanine green stained elastin biomaterial using a q-switched laser. In Laser-Tissue Interaction IX, volume 3254, pages 104-111, 1998.

[50] E.M. Spradling, R.J. Talbert, and J.A. Viator. Photoacoustic determination of the optical absorption coefficient of thermally coagulated blood. In Photons Plus Ultrasound: Imaging and Sensing 2008: The Ninth Conference on Biomedical Thermoacoustics, Optoacoustics, and Acousto-optics, volume 6856, 2008.

[51] J.A. Swearingen, S.H. Holan, M.M. Feldman, and J.A. Viator. Photoacoustic discrimination of vascular and pigmented lesions using classical and bayesian methods. Journal of Biomedical Optics, 15(1), 2010.

[52] R.J. Talbert, S.H. Holan, and J.A. Viator. Photoacoustic discrimination of viable and thermally coagulated blood using a two-wavelength method for burn injury monitoring. Physics in Medicine and Biology, 52(7):1815-1829, 2007.

[53] J.A. Viator, L.O. Svaasand, G. Aguilar, B. Choi, and J.S. Nelson. Photoacoustic measurement of epidermal melanin. In Biomedical Optoacoustics IV, volume 4960, pages 14-20, 2003. 
[54] Song $\mathrm{Hu}$, Konstantin Maslov, Vassiliy Tsytsarev, and Lihong V. Wang. Functional transcranial brain imaging by optical-resolution photoacoustic microscopy. Journal of Biomedical Optics, 14(4):040503-040503-3, 2009.

[55] K. Bhattacharyya, S. Mehta, and J. Viator. Optically absorbing nanoparticle mediated cell membrane permeabilization. Optics Letters, 37(21):4474-4476, 2012.

[56] Daniel Courjon. Near-Field Microscopy and Near-Field Optics. Imperial College Press, 2003.

[57] A.W. Snyder and J.D. Love. Optical waveguide theory. Science paperbacks. Chapman and Hall, 1983.

[58] A. Rosencwaig. Photoacoustic spectroscopy. Annual Review of Biophysics and Bioengineering, 9:31-54, 1980.

[59] J.A. Viator, S.L. Jacques, and S.A. Prahl. Depth profiling of absorbing soft materials using photoacoustic methods. IEEE Journal on Selected Topics in Quantum Electronics, 5(4):989-996, 1999.

[60] T. Hinoue, Y. Shimahara, and Y. Yokoyama. Photoacoustic observation of solid-liquid interface by means of total internal reflection technique. Chemistry Letters, 12(2):225-228, 1983.

[61] T. Hinoue, Y. Shimahara, H. Murata, and Y. Yokoyama. Photoacoustic spectrometry by total internal reflection technique: dependence of photoacoustic signal intensity on concentration and optical path. Bunseki Kagaku, 33:E459E466, 1984. 
[62] T. Hinoue, H. Murata, and Y. Yokoyama. Photoacoustic spectrometry coupled with total internal reflection technique: theory and experiment. Analytical Sciences, 2:401-406, October 1986.

[63] T. Hinoue, H. Murata, M. Kawabe, and Y. Yokoyama. Effects of thermal diffusion and solvent materials on photoacoustics signals in total internal reflection technique. Analytical Sciences, 2:407-410, October 1986.

[64] T. Hinoue, M. Kawabe, and Y. Yokoyama. Measurement of concentration profile of dye at glass-solution interface by photoacoustic spectrometry coupled with total internal reflection technique. Chemical Society of Japan, 60:3811-3813, 1987.

[65] T. Iwasaki, T. Sawada, H. Kamada, A. Fujishima, and K. Honda. Observation of semiconductor electrode-dye solution interface by means of fluorescence and laser-induced photoacoustic spectroscopy. The Journal of Physical Chemistry, 83(16):2142-2145, 1979.

[66] P. R. Muessig and G. J. Diebold. Total internal reflectance optoacoustic spectroscopy. Journal of Applied Physics, 54(8):4251-4253, 1983.

[67] T. Inagaki, K. Kagami, and E.T. Arakawa. Photoacoustic observation of nonradiative decay of surface plasmons in silver. Physical Review B, 24(6):3644-3646, September 1981.

[68] T. Inagaki, K. Kagami, and E.T. Arakawa. Photoacoustic study of surface plasmons in metals. Applied Optics, 21(5):949-954, 1982. 
[69] T. Inagaki, Y. Nakagawa, E.T. Arakawa, and D.J. Aas. Photoacoustic determination of radiative quantum efficiency of surface plasmons in silver films. Physical Review B, 26(12):6421-6430, 1982.

[70] H. Talaat and H.D. Dardy. Photoacoustic study of the interaction of surface plasmons and monolayers of dye molecules. In 1983 Ultrasonics Symposium, volume 2, pages 700-703, Atlanta, Ga, USA, 1983. IEEE, New York, NY, USA.

[71] B. Rothenhausler, J. Rabe, P. Korpiun, and W. Knoll. On the decay of plasmon surface polaritons at smooth and rough ag-air interfaces: A reflectance and photo-acoustic study. Surface Science, 137(1):373-383, 1984.

[72] C. S. Jung, G. Park, and Y. D. Kim. Photoacoustic determination of field enhancement at a silver surface arising from resonant surface plasmon excitation. Applied Physics Letters, 47(11):1165-1167, 1985.

[73] H. Talaat, J.A. Bucaro, W. Huang, and A.G. Macdiarmid. Photoacoustic detection of plasmon surface polaritons in heavily doped polyacetylene films. Synthetic Metals, 10(4):245-253, 1985.

[74] T. Inagaki, M. Motosuga, E.T. Arakawa, and J.P. Goudonnet. Coupled surface plasmons in periodically corrugated thin silver films. Physical Review B, 32(10):6238-6245, November 1985.

[75] T. Inagaki, J.P. Goudonnet, P. Royer, and E.T. Arakawa. Optical properties of silver island films in the attenuated-total-reflection geometry. Applied Optics, 25:3635-3639, October 1986. 
[76] J.P. Goudonnet, T. Inagaki, E.T. Arakawa, and T.L. Ferrell. Angular and polarization dependence of surface-enhanced raman scattering in attenuatedtotal-reflection geometry. Physical Review B, 36(2):917-921, July 1987.

[77] P. Royer, J. P. Goudonnet, T. Inagaki, G. Chabrier, and E. T. Arakawa. Photoacoustic study of the optical absorption of oblate silver spheroids in attenuatedtotal-reflection geometry. physica status solidi (a), 105(2):617-625, 1988.

[78] T. Hinoue, M. Kawabe, S. Doi, and Y. Yokoyama. Photoacoustic estimation of reflectivities at solid-liquid interfaces by using total internal reflection technique. Journal of the Surface Science Society of Japan, 10(2):47-52, 1989.

[79] S. Negm and H. Talaat. Surface plasmon resonance halfwidths as measured using attenuated total reflection, forward scattering and photoacoustics. Journal of Physics: Condensed Matter, 1(50):10201-10205, 1989.

[80] S. Negm and H. Talaat. Radiative and non-radiative decay of surface plasmons in thin metal films. Solid State Communications, 84(1-2):133-137, 1992.

[81] S. Negm and H. Talaat. Effect of intrinsic surface roughness and other decay processes on surface plasmon polariton resonance halfwidth. Ultrasonics Symposium, 1:509-514, October 1992.

[82] S. Negm, H. Talaat, and J. Pelzl. Photoacoustic study of surface roughness on thin evaporated gold films. In 1993 Ultrasonics Symposium, volume 2, pages 1259-1261, Baltimore, MD, USA, 1993. Publ by IEEE, Piscataway, NJ, United States. 
[83] K. Sathiyamoorthy, James Joseph, Chia Jin Hon, and Murukeshan V. Matham. Photoacoustic based surface plasmon resonance spectroscopy: an investigation. Proc. SPIE 8001, International Conference on Applications of Optics and Photonics, pages 80010K-80010K-8, 2011.

[84] K. Sathiyamoorthy, J. Joseph, C.J. Hon, and M.V. Matham. Photoacoustic based surface plasmon resonance spectroscopy: An investigation. In Proc. SPIE 8001, International Conference on Applications of Optics and Photonics, volume 8001, Braga, 2011.

[85] Y.S. Jiang, S.Y. Zhang, F. Qian, and H.P. Shao. Photoacoustic plasmon resonance spectra of langmuir-blodgett films. In 8th International Topical Meeting on Photoacoustic and Photothermal Phenomena, volume 4, pages 35-38, Guadeloupe, Fr, 1994. Editions de Physique, Les Ulis, France.

[86] Jiang, Y. S., Zhang, S. Y., Qian, F., and Shao, H. P. Photoacoustic plasmon resonance spectra of langmuir-blodgett films. J. Phys. IV France, 04:C7-35C7-38, 1994.

[87] T. Abdallah, S. Negm, and H. Talaat. Photoacoustic surface plasmon for the detection of nicotine. Egyptian Journal of Solids, 25(2):181-189, 2002.

[88] SPIE. Total internal reflection photoacoustic detection spectroscopy, 2010.

[89] B.S. Goldschmidt, A.S. Sudduth, E.B. Samson, P.J. Whiteside, K.D. Bhattacharyya, and J.A. Viator. Total internal reflection photoacoustic spectroscopy for the detection of beta-hematin. Journal of biomedical optics, 17(6):061212, 2012. 
[90] B.S. Goldschmidt, S. Mehta, J. Mosley, C. Walter, P.J.D. Whiteside, H.K. Hunt, and J.A. Viator. Photoacoustic measurement of refractive index of dye solutions and myoglobin for biosensing applications. Biomedical Optics Express, $4(11): 2463-2476,2013$.

[91] Yuze Sun, Jing Liu, Greg Frye-Mason, Shiou Jyh Ja, Aaron K. Thompson, and Xudong Fan. Optofluidic ring resonator sensors for rapid dnt vapor detection. Analyst, 134:1386-1391, 2009.

[92] M. Zourob, A. Simonian, J. Wild, S. Mohr, Xudong Fan, I. Abdulhalim, and N. J. Goddard. Optical leaky waveguide biosensors for the detection of organophosphorus pesticides. Analyst, 132:114-120, 2007.

[93] Harshini Mukundan, Aaron S. Anderson, W. Kevin Grace, Karen M. Grace, Nile Hartman, Jennifer S. Martinez, and Basil I. Swanson. Waveguide-based biosensors for pathogen detection. Sensors, 9(7):5783-5809, 2009.

[94] H. P. Lehr, M. Reimann, A. Brandenburg, G. Sulz, and H. Klapproth. Real-time detection of nucleic acid interactions by total internal reflection fluorescence. Analytical Chemistry, 75(10):2414-2420, 2003.

[95] Yuze Sun, Jing Liu, Daniel J. Howard, Greg Frye-Mason, Aaron K. Thompson, Shiou-jyh Ja, and Xudong Fan. Rapid tandem-column micro-gas chromatography based on optofluidic ring resonators with multi-point on-column detection. Analyst, 135:165-171, 2010. 
[96] J.T. Gohring, P.S. Dale, and X. Fan. Detection of her2 breast cancer marker using the opto-fluidic ring resonator biosensor. Sensors and Actuators, B: Chemical, 146(1):226 - 230, 2010.

[97] J.D. Suter, Y. Sun, D.J. Howard, J.A. Viator, and X. Fan. Integration of optofluidic microring resonator lasers for lab-on-a-chip development. In Photonic Fiber and Crystal Devices: Advances in Materials and Innovations in Device Applications II, volume 7056, 2008.

[98] J.D. Suter, Y. Sun, D.J. Howard, J.A. Viator, and X. Fan. Pdms embedded opto-fluidic microring resonator lasers. Optics Express, 16(14):10248-10253, 2008.

[99] Teruo Hinoue, Masaaki Kawabe, and Yu Yokoyama. Measurement of concentration profile of dye at glass-solution interface by photoacoustic spectrometry coupled with total internal reflection technique. The Chemical Society of Japan, pages 3811-3813, October 1987.

[100] Teruo Hinoue, Hideo Murata, and Yu Yokoyama. Photoacoustic spectrometry coupled with total internal reflection technique: Theory and experiment. Analytical Sciences, 2:401-406, October 1986.

[101] N. J. Harrick. Internal Reflection Spectroscopy. Harrick Scientific Corporation, 1987.

[102] G. Paltauf, H. Schmidt-Kloiber, and H. Guss. Light distribution measurements in absorbing materials by optical detection of laser-induced stress waves. Applied Physics Letters, 69(11):1526-1528, 1996. 
[103] L. Florens, M. Washburn, N. Muster, D. Wolters, M. Gardner, R. Anthony, D. Haynes, K. Moch, J. Sacci, A. Witney, N. Grainger, A. Holder, Y. Wu, J. Yates III, and D. Carucci. A proteomic view of the malaria parasite life cycle. In ASMS 50th Anniversary Volume, pages 59-60, 2002.

[104] Y.M. Serebrennikova, J. Patel, and L.H. Garcia-Rubio. Interpretation of the ultraviolet-visible spectra of malaria parasite plasmodium falciparum. Applied Optics, 49(2):180-188, 2010.

[105] Edward B Samson, Benjamin S Goldschmidt, Paul J D Whiteside, Amanda S M Sudduth, John R Custer, Brenda Beerntsen, and John A Viator. Photoacoustic spectroscopy of beta-hematin. Journal of Optics, 14(6):065302, 2012.

[106] T. Egan, W. Mavuso, and K. Ncokazi. The mechanism of beta-hematin formation in acetate solution. parallels between hemozoin formation and biomineralization processes. Biochemistry, 40:204-213, 2001.

[107] J.A. Viator. Characterization of Photoacoustic Sources in Tissue Using Time Domain Measurements. PhD thesis, Oregon Graduate Institute of Science \& Technology, 3181 S.W. Sam Jackson Park Rd. Portland, Oregon 97239-3098, August 2000 .

[108] A. Leung, P.M. Shankar, and R. Mutharasan. A review of fiber-optic biosensors. Sensors and Actuators, B: Chemical, 125(2):688-703, 2007.

[109] V.V.R. Sai, T. Kundu, C. Deshmukh, S. Titus, P. Kumar, and S. Mukherji. Label-free fiber optic biosensor based on evanescent wave absorbance at 280 nm. Sensors and Actuators, B: Chemical, 143(2):724-730, 2010. 
[110] M.D. DeGrandpre and L.W. Burgess. Long path fiber-optic sensor for evanescent field absorbance measurements. Analytical Chemistry, 60(23):2582-2586, 1988.

[111] V. Ruddy, B.D. MacCraith, and J.A. Murphy. Evanescent wave absorption spectroscopy using multimode fibers. Journal of Applied Physics, 67(10):60706074,1990 .

[112] P.H. Paul and G. Kychakoff. Fiber-optic evanescent field absorption sensor. Applied Physics Letters, 51(1):12-14, 1987.

[113] S. S. Saavedra and W. M. Reichert. Integrated optical attenuated total reflection spectrometry of aqueous superstrates using prism-coupled polymer waveguides. Analytical Chemistry, 62(20):2251-2256, 1990. PMID: 2268054.

[114] B. Choi, D. Jansen, and A. Welch. Acoustic-based measurements of material absorption coefficients: Relationship between laser pulse duration and stress confinement time. Journal of Applied Physics, 94(12):7826-7831, 2003.

[115] Allan Rosencwaig and Allen Gersho. Theory of the photoacoustic effect with solids. Journal of Applied Physics, 47(1):64-69, 1976.

[116] O. Sydoruk, O. Zhernovaya, V. Tuchin, and A. Douplik. Refractive index of solutions of human hemoglobin from the near-infrared to the ultraviolet range: Kramers-kronig analysis. Journal of Biomedical Optics, 17(11), 2012.

[117] A S Laurence, G K Vanner, W Collins, and P M Hopkins. Serum and urinary myoglobin following an aborted malignant hyperthermia reaction. Anaesthesia, 51(10):958-961, Oct 1996. 
[118] A. S. Laurence. Serum myoglobin and creatine kinase following surgery. British Journal of Anaesthesia, 84(6):763-766, 2000.

[119] Eugene Hecht. Optics (4th Edition). Addison Wesley, 4 edition, August 2001.

[120] L.V. Wang. Multiscale photoacoustic microscopy and computed tomography. Nature Photonics, 3(9):503-509, 2009.

[121] D. McCormack, K. Bhattacharyya, R. Kannan, K. Katti, and J.A. Viator. Enhanced photoacoustic detection of melanoma cells using gold nanoparticles. Lasers in Surgery and Medicine, 43(4):333-338, 2011.

[122] C.M. Hernandez, T.W. Murray, and S. Krishnaswamy. Photoacoustic characterization of the mechanical properties of thin films. Applied Physics Letters, 80(4):691-693, 2002.

[123] J.B. Kinney and R.H. Staley. Applications of photoacoustic spectroscopy. In Annual Review of Materials Science, volume 12, pages 295-321. Annu Rev Inc, Palo Alto, Calif, USA, 1982.

[124] Y.C. Shen, S.Y. Zhang, Y.S. Jiang, R. Zhu, and Y. Wei. Angular resonance absorption spectra of langmuir-blodgett films studied by the photoacoustic technique. Thin Solid Films, 248(1):36-40, 1994.

[125] Minghua Xu, Shuyi Zhang, and T. Inagaki. Investigation of optical resonance absorption on bigratings by photoacoustic angular spectroscopy. Shengxue Xuebao/Acta Acustica, 25(5):440-444, 2000. 
[126] Y. Jiang, S. Zhang, H. Shao, and C. Yuan. Optical properties of langmuirblodgett films investigated by a photoacoustic technique. Applied Optics, 34(1):169-173, 1995.

[127] Ultrasonics International. Photoacoustic resonance absorption spectra of langmuir-blodgett films, July 1993.

[128] R. W. Stineman. A consistently well-behaved method of interpolation. Creat. Comp., pages 54-57, July 1980.

[129] T. Hinoue, G. Imamura, and Y. Yokoyama. Study of the adsorption layer at the glass-dye solution interface by variable incidence-angle internal-reflection spectrometry. Bulletin of the Chemical Society of Japan, 66(12):3680-3685, 1993.

[130] P. Hess. Photoacoustic, Photothermal and Photochemical Processes at Surfaces and in Thin Films. Springer-Verlag, 1989.

[131] A. Rosencwaig. Photoacoustics and Photoacoustic Spectroscopy. Chemical Analysis: A Series of Monographs on Analytical Chemistry and Its Applications. Wiley, 1980.

[132] T. Coquil, E.K. Richman, N.J. Hutchinson, S.H. Tolbert, and L. Pilon. Thermal conductivity of cubic and hexagonal mesoporous silica thin films. Journal of Applied Physics, 106(3), 2009.

[133] Guy Rousseau, Bruno Gauthier, Alain Blouin, and Jean-Pierre Monchalin. Noncontact biomedical photoacoustic and ultrasound imaging. Journal of Biomedical Optics, 17(6):061217-1-061217-7, 2012. 
[134] Yi Wang, Chunhui Li, and Ruikang K. Wang. Noncontact photoacoustic imaging achieved by using a low-coherence interferometer as the acoustic detector. Opt. Lett., 36(20):3975-3977, Oct 2011.

[135] Armin Hochreiner, Johannes Bauer-Marschallinger, Peter Burgholzer, Bernhard Jakoby, and Thomas Berer. Non-contact photoacoustic imaging using a fiber based interferometer with optical amplification. Biomed. Opt. Express, 4(11):2322-2331, Nov 2013.

[136] K. Byrappa and B.V.S. Kumar. Characterization of zeolites by infrared spectroscopy. Asian Journal of Chemistry, 19(6):4933-4935, 2007.

[137] Ian N. Court and Frederick K. von Willisen. Frustrated total internal reflection and application of its principle to laser cavity design. Appl. Opt., 3(6):719-726, Jun 1964.

[138] D.W. Marquardt. An algorithm for least-squares estimation of nonlinear parameters. Journal of the Society for Industrial and Applied Mathematics, 11(2):431441, 1963.

[139] F.N. Dultsev. Investigation of the microporous structure of porous layers using ellipsometric adsorption porometry. Thin Solid Films, 458(1-2):137 - 142, 2004.

[140] Roy G. M. Kolkman, Erik Blomme, Ton G. van Leeuwen, Wiendelt Steenbergen, Kees A. Grimbergen, Gerard J. den Heeten, Tijl Cool, and Mattias Bilcke. Feasibility of noncontact piezoelectric detection of photoacoustic signals in tissue-mimicking phantoms. Journal of Biomedical Optics, 15(5):055011055011-4, 2010. 
[141] James A Blackburn. Modern instrumentation for scientists and engineers. Measurement Science and Technology, 13(10):1652, 2002. 


\section{VITA}

Benjamin S. Goldschmidt attended the University of Missouri in 2005 and received a B.S. in Biological Engineering in 2009. Benjamin started working with the photoacoustic effect under the guidance of Dr. John A. Viator in 2008. Mr. Goldschmidt has cofounded two business, Red Flag Biosensing LLC as well as Medical Photonics Industries to pursue commercial applications of the photoacoustic effect

and related technologies. In 2013, he received the Missouri Outstanding Graduate Student Award for his work in the Viator lab. Benjamin has continued by pursuing a $\mathrm{PhD}$ in Biological Engineering at the University of Missouri. 